\title{
On the Efficiency of Using Multiple Hops in Fixed Relay Based Wireless Networks
}

\author{
By \\ Adrian Florea, Bachelor of Applied Science
}

\author{
A thesis submitted to \\ The Faculty of Graduate Studies and Research \\ In partial fulfillment of \\ The requirements of the degree of
}

Master of Applied Science

Ottawa-Carleton Institute for Electrical and Computer Engineering

Department of Systems and Computer Engineering

Carleton University

Ottawa, Ontario

December 2004 


$\begin{array}{ll}\begin{array}{l}\text { Library and } \\ \text { Archives Canada }\end{array} & \begin{array}{l}\text { Bibliothèque et } \\ \text { Archives Canada }\end{array} \\ \begin{array}{l}\text { Published Heritage } \\ \text { Branch }\end{array} & \begin{array}{l}\text { Direction du } \\ \text { Patrimoine de l'édition }\end{array} \\ \begin{array}{l}\text { 395 Wellington Street } \\ \text { Ottawa ON K1A ON4 }\end{array} & \begin{array}{l}\text { 395, rue Wellington } \\ \text { Ottawa ON K1A ON4 } \\ \text { Canada }\end{array}\end{array}$

Your file Votre référence

ISBN: 0-494-00738-9

Our file Notre référence

ISBN: 0-494-00738-9

NOTICE:

The author has granted a nonexclusive license allowing Library and Archives Canada to reproduce, publish, archive, preserve, conserve, communicate to the public by telecommunication or on the Internet, loan, distribute and sell theses worldwide, for commercial or noncommercial purposes, in microform, paper, electronic and/or any other formats.

The author retains copyright ownership and moral rights in this thesis. Neither the thesis nor substantial extracts from it may be printed or otherwise reproduced without the author's permission.
AVIS:

L'auteur a accordé une licence non exclusive permettant à la Bibliothèque et Archives Canada de reproduire, publier, archiver, sauvegarder, conserver, transmettre au public par télécommunication ou par l'Internet, prêter, distribuer et vendre des thèses partout dans le monde, à des fins commerciales ou autres, sur support microforme, papier, électronique et/ou autres formats.

L'auteur conserve la propriété du droit d'auteur et des droits moraux qui protège cette thèse. $\mathrm{Ni}$ la thèse ni des extraits substantiels de celle-ci ne doivent être imprimés ou autrement reproduits sans son autorisation.
In compliance with the Canadian

Privacy Act some supporting forms may have been removed from this thesis.

While these forms may be included in the document page count, their removal does not represent any loss of content from the thesis.
Conformément à la loi canadienne sur la protection de la vie privée, quelques formulaires secondaires ont été enlevés de cette thèse.

Bien que ces formulaires aient inclus dans la pagination, il n'y aura aucun contenu manquant.

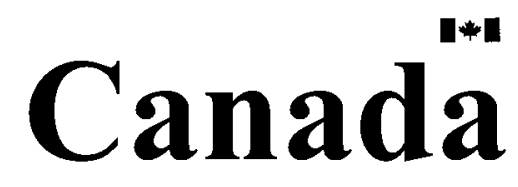




\begin{abstract}
This thesis is about deployment strategies for $4 \mathrm{G}$ cellular networks using fixed relays. We argue that while relays will surely be an essential component of future $4 \mathrm{G}$ systems, their indiscriminate use is an inefficient way to allocate the limited radio resources available. The most efficient method to allocate the radio license is when the number of relays is minimized, while still achieving the desired coverage objectives. Thus, the focus of this work is to determine the optimal number and locations of fixed radio relay stations forming multi-hop links, such a way the right balance between achieving the functional requirements and assigning minimum resources is achieved. Under the assumption of using orthogonal channels for the intermediate hops, and using the spectral efficiency as a metric, it is shown that the optimal relay locations are at equal intervals along the straight line between source and destination. The Multi-Hop Criterion (MHC) to determine in which conditions a single-hop fixed radio link can be efficiently (from a spectral efficiency perspective) replaced with a chain of (multi-hop) links is introduced. Since a routing method is required to take advantage of any multi-hop architecture over a two-dimensional array of relays, four routing algorithms are devised and compared: direct single hop to destination, multi-hop using shortest possible hops, multi-hop using MHC to decide the number of hops, and exhaustive search for the route with smallest cost. Through computer simulations it is shown that the routing algorithm based on the MHC always achieves a spectral efficiency performance which is close to or equals the optimal (exhaustive search), outperforming both "single-hop" and "short-hops" schemes.
\end{abstract}




\section{ACKNOWLEDGEMENTS}

I am deeply indebted to Dr. Halim Yanikomeroglu for the unconditional support he offered me in the past two years. His guidance and suggestions have made this work possible. To my wife Mihaela and my daughter Alexandra, my deepest gratitude for their patience and help through this period. 


\section{Table of Contents}

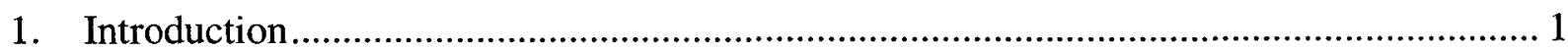

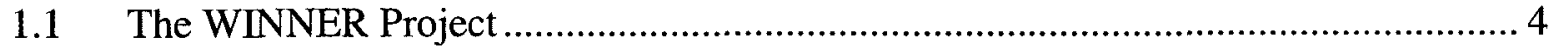

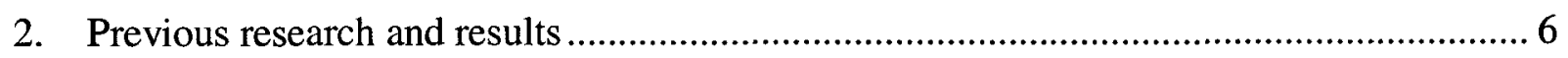

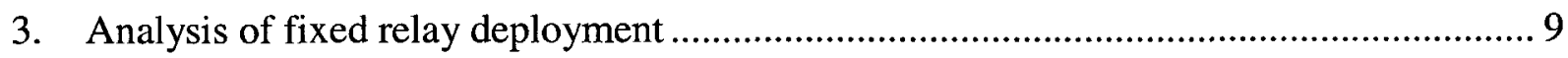

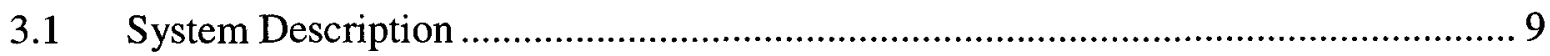

3.2 Radio Resource Allocation to Access and Feeder Systems................................... 11

3.2.1 A Different View Of The Same Problem ................................................. 16

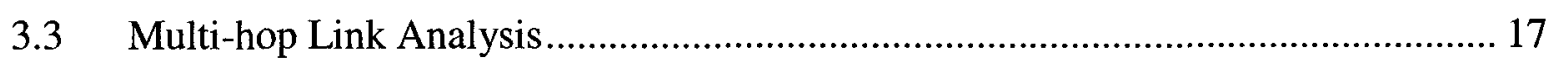

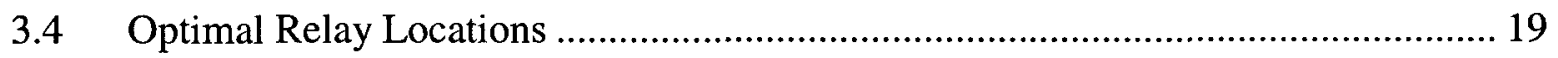

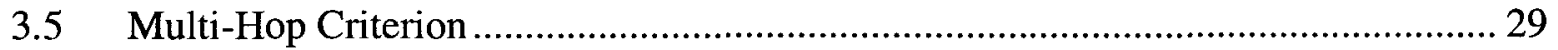

3.5.1 Reduced path loss exponent for shorter links ........................................ 31

3.6 System Model Considering Shadowing ............................................................ 34

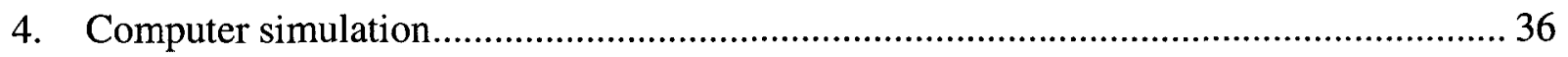

4.1 System Model for Computer Simulation ........................................................ 36

4.1.1 Method 1 - Single-hop transmission ....................................................... 41

4.1.2 Method 2 - Greedy multi-hop transmission .......................................... 42

4.1.3 Method 3 - Multi-Hop transmission using Multi-Hop Criterion (MHC)........ 44

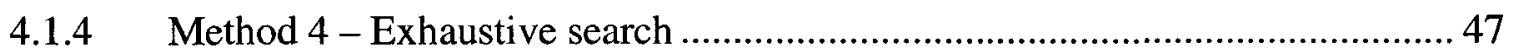

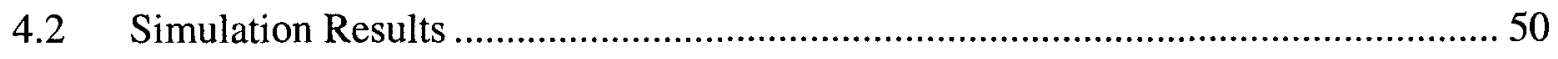

4.2.1 Same path loss exponent for all links ................................................. 51

4.2.2 Reduced path-loss exponent value for short links ...................................... 56

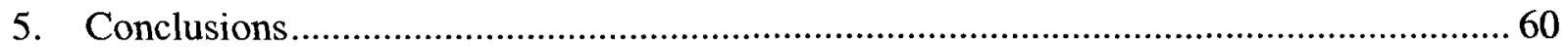

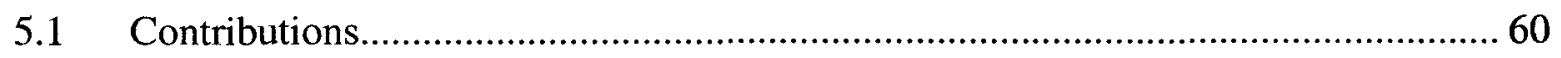

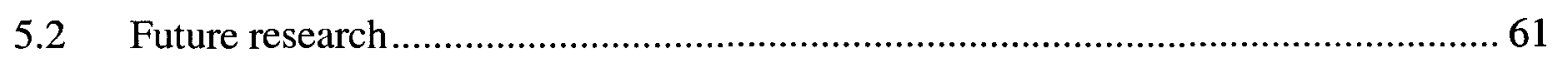

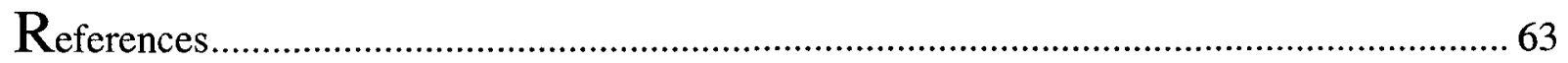




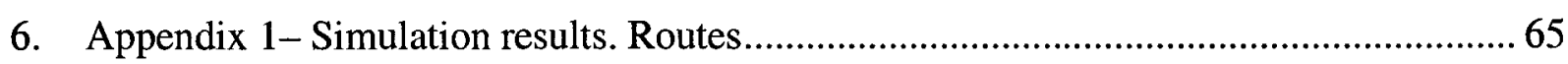

\section{Table of figures}

Figure 1. Proposed system architecture for a 4G cellular system ............................................... 10

Figure 2. Required license versus the number of FRS in each BS ............................................... 14

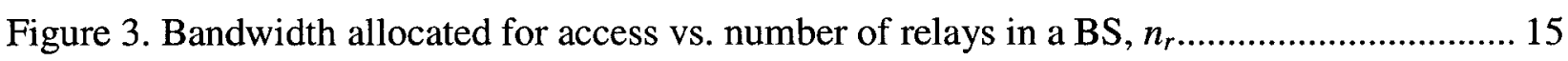

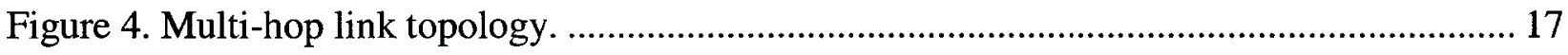

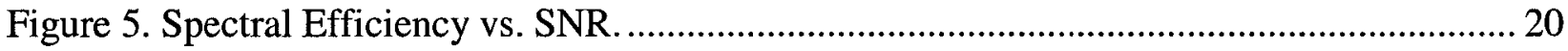

Figure 6. Message Transfer Time vs. Hop Length ................................................................... 24

Figure 7. Geometric possible locations for relays in a two-hop link …………………………... 25

Figure 8. The ratio between message transfer times of a two-hop link and the link with the two

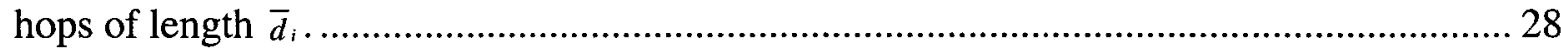

Figure 9. SNR values above which the single-hop has better spectral efficiency compared to any

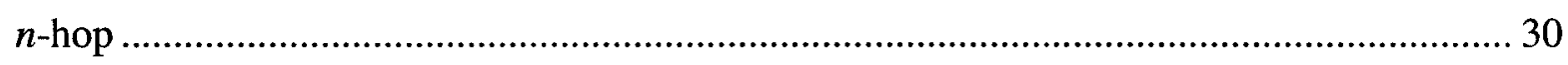

Figure 10. MHC break-even SNR values. Intermediate hops have a ........................................... 33

Figure 11. Reciprocal Gaussian random variable ………....................................................... 35

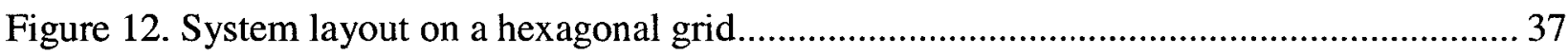

Figure 13. Example of network topology, direct single-hop transmission ..................................... 41

Figure 14. Greedy multi-hop transmission - algorithm flowchart ............................................. 43

Figure 15. Example of network topology, greedy multi-hop transmission ....................................4 44

Figure 16. Algorithm to determine number of hops using MHC ................................................. 45

Figure 17. Multi-hop transmission using MHC - algorithm logical flowchart ............................. 46

Figure 18. Network topology - transmission using MHC ............................................................ 47

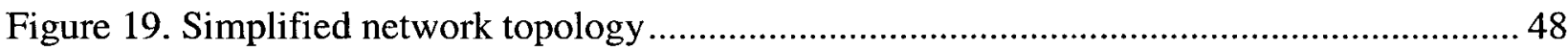

Figure 20. Algorithm flowchart - exhaustive route search ......................................................... 49

Figure 21. Network topology, exhaustive search..................................................................... 50

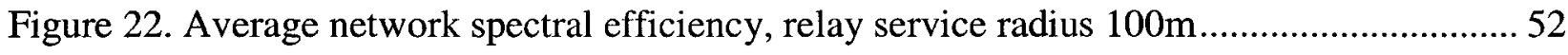

Figure 23. Average network spectral efficiency, relay service radius $150 \mathrm{~m} . . . \ldots \ldots \ldots \ldots \ldots \ldots \ldots \ldots \ldots \ldots \ldots . . .53$ 
Figure 24. Average Spectral Efficiency vs. Standard deviation

Figure 25. Average network spectral efficiency, relay service radius $100 \mathrm{~m}, \alpha=0.8 \ldots \ldots \ldots \ldots \ldots .57$

Figure 26. Average network spectral efficiency, relay service radius $150 \mathrm{~m}, \alpha=0.8 \ldots \ldots \ldots \ldots \ldots . . .58$

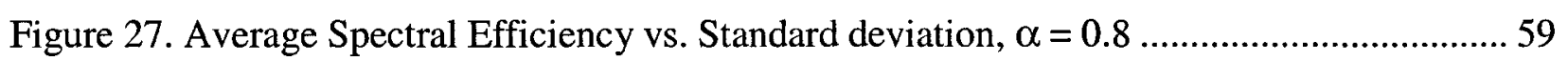




\section{List of Acronyms}

ASE

BS

FRS

MHC

MT

MTT

WAS

WFS

GIS
Average network Spectral Efficiency

Base Station

Fixed Relay Station

Multi Hop Criterion

Mobile Terminal

Message Transfer Time

Wireless Access System

Wireless Feeder System

Geographic Information Systems

\section{List of Symbols}

$\alpha \quad$ Path loss exponent reduction coefficient

A Total available frequency license

$B \quad$ Channel bandwidth

$b_{a} \quad$ Bandwidth allocated for access portion to each FRS

$b_{A} \quad$ Total resources allocated for access for all FRS in one sector

$b_{f} \quad$ Amount of resources allocated for feeder portion to each FRS

$b_{F} \quad$ Total resources allocated for feeder for all FRS in one sector

C Aggregate throughput for each FRS

$D \quad$ Distance source - destination

$d_{0} \quad$ Reference close in distance for propagation model

$d_{i} \quad$ Length hop $i$

F Carrier frequency

$\gamma \quad$ SNR direct link

$\gamma_{i} \quad$ SNR for hop $i$ 
$G_{R} \quad$ Gain receive antenna

$G_{T} \quad$ Gain transmit antenna

$\eta_{a} \quad$ Spectral efficiency for the WAS

$\eta_{f} \quad$ Spectral efficiency for the WFS

$\eta_{i} \quad$ Spectral efficiency for hop $i$

$K_{I} \quad$ Proportionality constant

$K_{2} \quad$ Proportionality constant

$\lambda \quad$ Carrier radio wavelength

$M \quad$ Message size

$N \quad$ WAS (relay) cluster Size for WAS ( $\mathrm{N}=3$ in Figure 1)

$n \quad$ Number of hops

$N F \quad$ Receiver noise factor

$n_{r} \quad$ Number of FRSs "feeded" from one BS

$p \quad$ Path loss exponent

$P_{N} \quad$ Noise power

$P_{T} \quad$ Transmit power

$R \quad$ BS service radius

$r \quad$ Relay coverage radius

$R_{0} \quad$ Source station

$R_{n} \quad$ Destination station

$S \quad$ Number of sectors at each BS of WFS

$s \quad$ Feeder frequency reuse factor at BS

$\sigma \quad$ Standard Deviation

$T$. Message transfer time for entire multi-hop link

$t_{i} \quad$ Message transfer time for hop i 


\section{INTRODUCTION}

It is generally accepted that the architecture of the present day cellular networks can not meet the stringent requirements envisioned for $4^{\text {th }}$ generation $(4 \mathrm{G})$, or beyond IMT-2000, cellular systems. On one side, the data rates envisioned for user services under $4 \mathrm{G}$ systems are orders of magnitude higher than what is presently considered as the state of the art. On the other side, an almost chronic insufficiency of radio spectrum for the new services will limit the availability of additional resources for the deployment of $4 \mathrm{G}$ systems. It is likely that new radio spectrum licenses for $4 \mathrm{G}$ systems will be allocated well above the operating frequency bands of the present systems, perhaps in $5 \mathrm{GHz}$ or even $7 \mathrm{GHz}$ bands. At these higher frequencies the radiopropagation is much more susceptible to impairments generated by non line of sight conditions. The obvious conclusion is the cellular network infrastructure density must be dramatically increased to be able to provide ubiquitous services with the high data rates envisioned for $4 \mathrm{G}$ systems. This would involve installing many new base stations, and expanding the backbone infrastructure to connect the new cell sites to the packet switched public network. However, it is not clear how the large capital costs required for such expansion could be recovered. The market penetration of cellular services is already closing onto $90 \%$ levels in many developed countries, and not much growth can be expected in this segment. In addition, increasing the density of cell sites will create a large additional amount of available capacity, well in excess of requirements, given the saturation (already) reached in market penetration. In summary, what is needed is a cost effective solution enabling ubiquitous deployment of very high speed services, which in the same time would not necessarily add extra capacity to the system.

Although recent technological advances in signal processing techniques (such as interference cancellation algorithms) and antenna architectures (generally referred to as smart antennas - such as MIMO and adaptive antennas) enabled dramatic increases in link throughput and spectral 
efficiency, these will not be sufficient by themselves to resolve the problem stated above [1]. Although incorporation of these concepts in future wireless systems is essential, it may be infeasible to deploy complex antenna systems at wireless terminals. Besides, in the presence of heavy shadowing, even the smartest antenna can not be of much help.

Economically feasible solutions are likely to be based on some form of multi-hop relaying allowing coverage extension and reducing the required number of expensive cell sites. This being said, the large interest of the research community in the specification and development of 4G systems has not yet been concluded in agreement over what particular network architectures or deployment strategies is to be followed.

Two different topological classes of multi-hop relaying are widely considered in the literature [2-3]. Ad-hoc or mobile relaying uses the mobile terminals as relays to pass along data packets between base stations and mobile terminals. This type of relaying has the advantage of reduced infrastructure cost and good potential coverage due to the large number of terminals which can also double as relays, however it has the drawback of increased cost and complexity for the user equipment, as well as reduced battery life, complex and likely inefficient routing protocols for radio packets, and also a fundamental incertitude regarding the degree of service offered to users (there is no guarantee of good coverage).

Multi-hop relaying with fixed relays is based on fixed relay stations deployed as part of the network infrastructure. Their incremental cost is offset by reduced requirements on the mobile terminals, and by the simplicity and efficiency of the radio protocols involved. The fixed relay stations are part of the entire cellular network infrastructure, therefore their deployment will be an integral part of the network planning, design and deployment process. Deploying fixed relays with the required high geographical density would still present financial and technical challenges; engineering weatherized low cost devices with a small footprint, reduced power consumption, easy to install and commission, and in the same time able to provide high reliability and security, are no easy tasks, which would have to be solved in coming years. 
Relaying systems can also be classified as "digital" (also referred as "regenerative relaying" or "decode and forward relaying"), or analog ("non-regenerative relaying", "amplify and forward"). Digital relays have the main advantage of not propagating the noise along a multi-hop link chain. On the other side, analog relays could be either less expensive to build (less signal processing involved), or they could allow MRC to be performed on original and relayed messages, enabling so called "multi-hop path diversity".

It is necessary to establish strategies and methods for efficient deployment of fixed relay stations, in such a way the overall cost of the network is minimized. Efficient radio resource allocation to network elements is a critical part of the overall network cost optimization effort.

One of the open questions regarding the deployment of wireless networks using fixed relays is the optimal number of hops between the source and destination radio stations. It is important to be able to decide with reasonable accuracy in what conditions it is more advantageous to send a signal directly to destination (may be by increasing the allocated transmit power, bandwidth or time) or route the same signal over a number of relay stations, each using much less resources compared to the replaced link. Due to their ambitious requirements, the $4 \mathrm{G}$ systems will most likely use solutions with at least two hops (whenever necessary), one of the hops being between the fixed relay and the mobile terminal. The remaining radio link going back to the base station, referred to as the "feeder system", is comprised of a set of fixed relays interconnected through radio links, and can have one or more hops. While it is generally accepted that there is a clear requirement for at least one relay between the base station (BS) and the mobile terminal (MT), the optimal number of hops of the feeder portion of the $4 \mathrm{G}$ wireless system (a number equal with or larger than one) is the focus of our research. 


\subsection{The WINNER Project}

WINNER - Wireless world Initiative New Radio, https://www.ist-winner.org/ - is a consortium of 38 partners ${ }^{1}$ co-ordinated by Siemens working towards enhancing the performance of mobile communication systems. The improvements of radio transmission to be explored by WINNER are crucial for enabling new mobile services and applications anytime and anywhere.

${ }^{1}$ The list of WINNER partners includes: Siemens AG, Munich; Aalborg University, Aalborg Denmark; Alcatel SEL AG, Stuttgart Germany; Carleton University, Ottawa, Ontario, Canada; Centre Tecnològic de Telecomunicacions de Catalunya, Barcelona, Spain; Chalmers University of Technology, Gothenburg Sweden; China Academy of Telecommunication Research, Beijing PR China; Deutsches Zentrum für Luft- und Raumfahrt (DLR) e.V., Oberpfaffenhofen, Germany; DoCoMo Communications Laboratories Europe $\mathrm{GmbH}$, Munich, Germany; Elektrobit Ltd, Oulu Elektrobit Ltd, Oulu Finland; Ericsson AB, Stockholm Ericsson AB, Stockholm Sweden; Ericsson Eurolab Deutschland GmbH, Aachen, Germany; Heidelberg European Institute for Research and Strategic Studies in Telecommunications GmbH, Heidelberg Germany; France Telecom S.A. Paris France; Fujitsu Laboratories of Europe Ltd, Hayes, Middlesex United Kingdom; Helsinki University of Technology, Espoo Finland; IBM United States; Kungl Tekniska Högskolan (Royal Institute of Technology) - KTH, Stockholm Sweden; Lucent Technologies Network Systems, UK Ltd, Swindon United Kingdom; Motorola S.A.S., Paris France; National Technical University of Athens, Athens Greece; Nokia Corporation, Espoo Finland; Philips Electronics UK Limited, Redhill United Kingdom; Portugal Telecom Inovacao S.A. Aveiro Portugal; Poznan University of Technology, Poznan Poland; RWTH Aachen University (ComNets) RWTH Aachen University (ComNets) Germany; Samsung Electronics UK Ltd, Staines United Kingdom; Siemens Mobile Communications SPA, Milan Italy; Swiss Federal Institute of Technology Zurich, Zurich Switzerland; Technical Research Centre of Finland VTT, Oulu Finland; Technische Universität Dresden, Dresden, Germany; Technische Universität IImenau, IImenau, Germany; Telefónica Investigación y Desarrollo Socieded Anónima Unipersonal, Madrid Spain; University of Oulu - CWC, Oulu Finland; University of Surrey, Guildford United Kingdom; Vodafone Group Services Ltd, Newbury United Kingdom; Siemens Aktiengesellschaft Österreich, Vienna Austria; Nokia (China) Investment Co., Ltd., Beijing PR China; IBM Research GmbH, Rüschlikon, Switzerland Switzerland. 
ITU-R approved the Recommendation M.1645 [4] in June 2003 as a basis of the future activities. The assumptions regarding service delivery methods and performance targets used in the WINNER project are also based on this ITU-R recommendation.

The ITU-R vision for systems beyond $3 \mathrm{G}$ comprises two major paths:

- On one hand existing and evolving access systems will be integrated on a packetbased platform to enable cooperation and interworking of these systems in the sense "optimally connected anywhere, anytime".

- On the other hand the radio access system for new mobile access and new nomadic/local area wireless access will be developed to provide access with significantly improved performance compared to today's systems. The focus of the WINNER project is the development of this radio access system by taking into account the interworking with other systems.

The envisioned capabilities of the new components of future mobile and wireless communication systems were agreed with the following peak aggregate user data rates:

- up to approximately $100 \mathrm{Mbps}$ for the new mobile access and

- up to approximately $1 \mathrm{Gbps}$ for new nomadic / local area wireless access.

The WINNER project is organized in "workpackages". Workpackage 3 (WP3) - Radio Network - includes 5 tasks:

- Task 3.1 - Enhanced Conventional Radio Network Deployment Concepts

- Task 3.2 - Relay-based concepts with homogeneous RI

- Task 3.3 - Integration of Relay Based and Conventional Deployment Concepts and Development of a Harmonized RAN Protocol Approach

- Task 3.4 - Relay Based Concepts with Heterogeneous RIs

- Task 3.5 - System Level Traffic Performance Evaluation

The research in this thesis represents a portion of Carleton University's contribution to the WINNER project, Task 3.2. The work was supported in part by the Natural Sciences \& Engineering Research Council of Canada under participation in the WINNER project. 


\section{PREVIOUS RESEARCH AND RESULTS}

The interest in multi-hop technology is not new. The packet relaying concept was introduced in the 1970s during research into packet radio networks (PRN) [5]. The aim of the early research efforts was focused on developing systems targeted for private radio networks (mostly applications for military or emergency systems) having improved reliability, anti-jamming characteristics, and extended coverage. When the requirements for a $4 \mathrm{G}$ cellular systems started to be defined and it became apparent that they create a tremendous stress on the "single-hop" link budget, the interest in relaying and multi-hop radio networks was renewed, this time with an emphasis on applications to commercial public cellular networks.

Laneman et al [6-7], based on the use of the classic relay channel, proposed a macrodiversity technique applicable to ad-hoc networks. A message broadcasted by the source is simultaneously received by the intended destination and an intermediate relay. The relay repeats the message towards the destination receiver, which performs MRC between the two copies of the same message.

General theoretical characterization for four different channel models - the decoded relaying multihop channel, the amplified relaying multihop channel, the decoded relaying multihop diversity channel, and the amplified relaying multihop diversity channel - are provided by Boyer et al in [8]. Expanding the model introduced in [6], in the case of the amplified relaying multihop diversity channel, the receiver of the destination and the intermediate relays is assumed to perform MRC between a multitude of copies of the same message arriving from the set of amplifying relays located "uphill" along a multihop chain. It is shown that the performance of amplified relaying, especially amplified relaying with multihop diversity, is generally better than that of decoded relaying, despite noise propagation. 
In [9], the authors compare the throughput of a multi-hop cellular network (MCN) with that of a single-hop cellular network ( $\mathrm{SCN}$ ), by analyzing the probability of packet collision in multihop and single-hop network architectures. In MCN architecture, it is considered that the mobile stations can relay messages between themselves and the base stations. Within the simplifying assumptions that multi-hop links are in straight lines and with equal hop lengths, and all radio links are implemented at the same data rate, it is shown that $\mathrm{MCN}$ has a superior throughput due to lower packet collision probability.

The effect of Rayleigh fading on a MPRN was analyzed in [10-11], in the context of transmission strategies based on different routing schemes, namely, Most Forward with Fixed Range (MFR), Nearest with Forward Progress (NFP), Minimal Angular Deviation (MAD), and Angular Deviation to Transmission Range Ratio (ARR). In each of these methods the routing decisions are based on various geometrical criteria which assume the mobile radio station has the geographical notion of "forward" toward the packet's destination (for each packet!) and backward from it. For example, the NFP method transmits to the nearest neighbor in the forward direction. Mobiles locations could be obtained by way of each mobile broadcasting its location at regular intervals, in a round robin fashion.

A multihop protocol and an opportunistic forwarding method, Multiuser Diversity Forwarding, are proposed in a recent work in [12]. The proposed MDF method consists in a synchronized probing and transmission, and a "fast precision" scheduling algorithm. The probing stage enables link rate adaptation prior to transmission. It uses a synchronized and time aligned process along all nodes in the network. Initially each node decides if it is to transmit or receive during the given time slot interval. Then, all transmitting nodes broadcast a pilot signal enabling neighboring receiving nodes to evaluate link quality and send back a probe response with the channel status parameters. The scheduling algorithm provides two degrees of freedom, allowing both the selection of the destination relay and channel rate adaptation. Computer simulations show that MDF performs significantly better than NFP in Rayleigh fading conditions [11]. 
In [13], the potential gains of a multi-hop link are analyzed starting from the Shannon capacity of the radio channel. Assuming orthogonal relaying channels, that the relays are equally spaced along the multi-hop link, and that the overhead increases linearly with the number of hops, it is concluded that a multi-hop link offers a positive system gain in specific radio conditions (low transmit power, large distance, large path loss exponent) only for a modest number of hops (up to four). The term oligohop is introduced, meaning a multi-hop link with a reduced number of hops ("oligo" means "a few" in Greek).

The effect of multi-hop relaying using fixed relays on the cellular network throughput is studied in [15]. Assuming a relaying scheme with loop-less trees routing, and using nonorthogonal channels (with frequency reuse), it is shown that the aggregate cell throughput for cellular fixed relay networks depends only on the number of tree roots, which can also be interpreted as the number of sectors of the central cell. Increasing the total number of relays beyond a certain limit does not increase the aggregate cell throughput.

In this paper we assume that the intermediate relays can be located anywhere, not only along straight lines at equal intervals, as in most of previous literature. Also, we take a novel approach by using the aggregate spectral efficiency of the multi-hop communication system as a costing factor. The most significant challenge ahead of the $4 \mathrm{G}$ cellular systems is the provision of quasi ubiquitous coverage with very high data rates. A promising solution for surpassing this difficulty and extending the coverage of the classical cellular network is by using relays. The additional radio power introduced in the network by relays comes from the "wall plug" and should not be added to the radio resource costs. More significant than the relay consumed power is the effect of the relaying schemes over the aggregate end-to-end spectral efficiency. Higher overall spectral efficiency allows better use of the available spectrum license - the most expensive asset of the cellular operator. 


\section{ANALYSIS OF FIXED RELAY DEPLOYMENT}

\subsection{System Description}

We consider a possible system architecture for a $4 \mathrm{G}$ cellular network, as shown in Figure 1.

The network is organized into two overlaid systems as follows:

- The wireless access system (WAS) has the role of providing wireless connectivity at the required bit rate and grade of service to the user terminals; the access system is composed of

- mobile user terminals (MT)

- fixed radio stations (relays and base stations) directly connected to the mobile user terminals

- radio resources allocated to the wireless access system

- The wireless feeder system (WFS) has the role of transporting the traffic generated by the access system elements to / from the wired backbone network; the wireless feeder system is composed of:

- fixed relay stations

- base stations

- radio resources allocated to the wireless feeder system

Remarks:

1. For the purposes of this study, the logical difference between a BS and a fixed relay station (FRS) essentially is that the BS has a wired connection to the backbone network, while the FRS does not. For the purposes of the present study, the BSs and MTs are terminating points for the traffic, while the FRSs have a "bridging" role. 


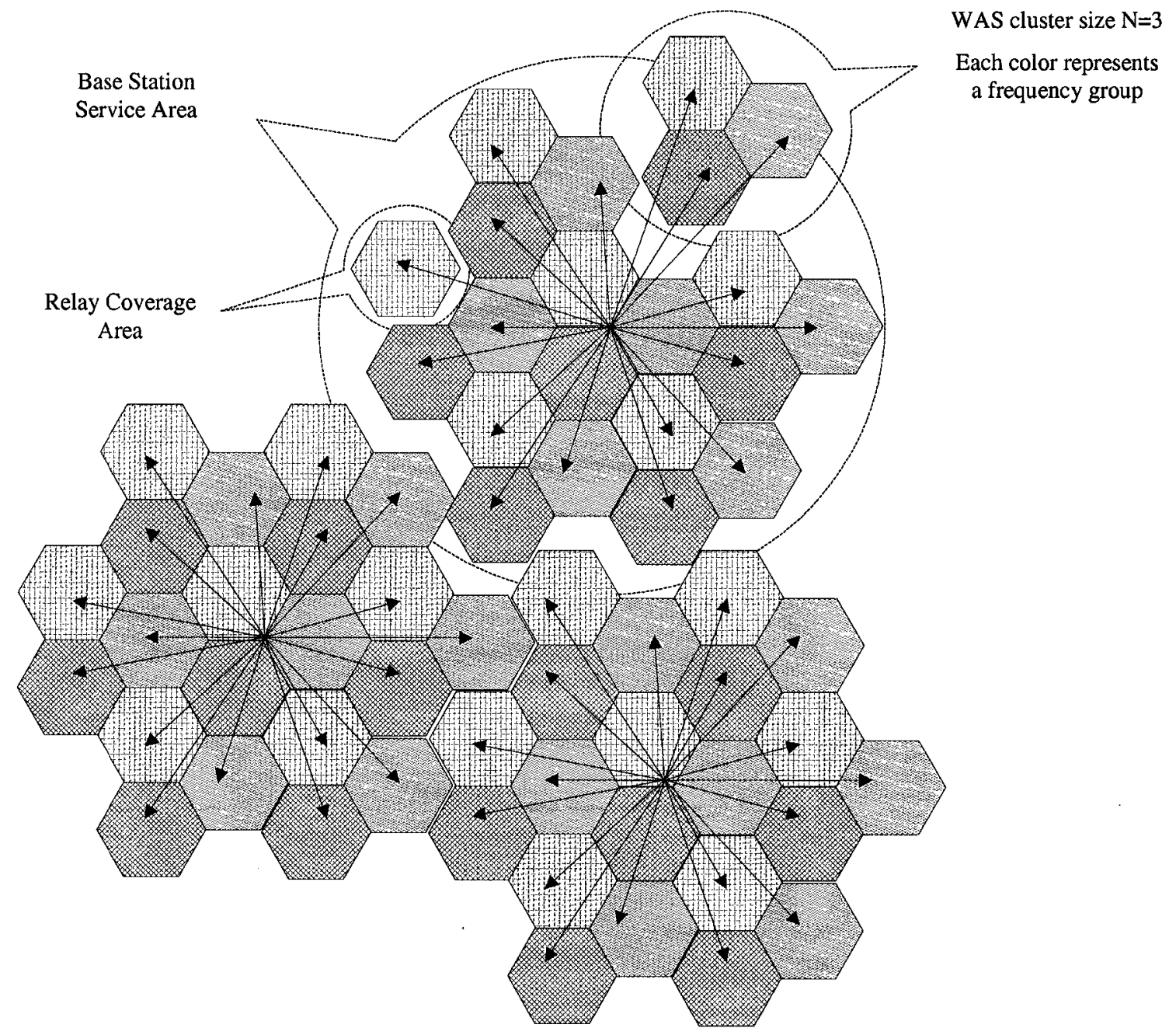

Figure 1. Proposed system architecture for a 4G cellular system

2. The main cost associated with a base station (BS) is assumed to be the cost of the wired data connection to the backbone or Internet (this involves a potentially considerable initial capital cost, as well as a monthly recurring cost). This is the reason of employing FRS instead of BS, as a means to reduce the total capital and operational costs of the network.

3. The wireless access system (WAS) and the wireless feeder system (WFS) could use similar physical/ MAC protocols and I/F interfaces, in which case the entire system is homogeneous, or use dissimilar physical/ MAC protocols and I/F interfaces, in which case we have a heterogeneous system [13]. 
A number of FRS is connected to one BS, which can be either omnidirectional or sectorized

with $S$ sector antennas. The maximum possible radius of the BS is determined by the link budget of the WFS.

In the present study the FRS are assumed to be non - sectorized (beside the directional antenna(s) used for the feeder system, each FRS has one antenna for access, which can be directional or omnidirectional, as dictated by the specific local conditions). The FRS maximum coverage radius is determined by the WAS link budget.

\subsection{Radio Resource Allocation to Access and Feeder Systems}

We analyze here the radio resource allocation strategy for the system architecture introduced in the previous section, particularly the division of available resources between the feeder and access portions of the network. By resources we understand bandwidth and/or time slot allocated to a particular network element. In the following discussion, the terms "bandwidth" and "radio resource" are used interchangeably.

For simplicity, we assume argument that the feeder link is implemented in one single hop (direct radio link between each FRS and BS). For the purposes of this discussion, the single hop assumption does not limit the generality of the result.

The following notations are made in the context of the system architecture presented in Figure 1.

$$
\begin{aligned}
& N=\text { WAS (relay) cluster Size for WAS }(N=3 \text { in Figure 1) } \\
& S=\text { number of sectors at each BS of WFS } \\
& \eta_{a}=\text { average spectral efficiency for the WAS } \\
& \eta_{f}=\text { spectral efficiency for the WFS }
\end{aligned}
$$


$s=$ feeder frequency reuse factor at BS (this measure is equivalent with $N$ for WAS; represents how many frequency groups are needed for the feeder system for all $S$ sectors of the BS).

$n_{r}=$ number of FRSs "fed" from one BS

$b_{a}=$ amount of resources allocated for access portion to each FRS (normalized)

$b_{A}=$ total resources allocated for access for all FRS in one sector

$b_{f}=$ amount of resources allocated for feeder portion to each FRS (normalized)

$b_{F}=$ total resources allocated for feeder for all FRS in one sector

The number of FRS for one sector is $n_{r} / S$.

For each FRS, the maximum data rate at the link layer must be the same for both the access and feeder links (otherwise very large buffers would be required at FRSs):

$$
b_{a}=b_{f} \cdot \frac{\eta_{f}}{\eta_{a}}
$$

The total frequency spectrum required for the entire system is composed of the spectrum required for the WFS and spectrum required for WAS. This is expressed in the normalized form as:

$$
\begin{aligned}
& b_{F}=\frac{n_{r}}{S} b_{f} s \\
& b_{A}=N b_{a} \\
& \frac{n_{r}}{S} b_{f} s+N b_{a}=1
\end{aligned}
$$

From (1),(2) we obtain the expressions for bandwidth allocated for each FRS for feeder and access, respectively:

$$
b_{f}=\frac{1}{N \frac{\eta_{f}}{\eta_{a}}} \cdot \frac{1}{1+\frac{n_{r} s}{N S \frac{\eta_{f}}{\eta_{a}}}}
$$




$$
b_{a}=\frac{1}{N} \cdot \frac{1}{1+\frac{n_{r} s}{N S \frac{\eta_{f}}{\eta_{a}}}} .
$$

The total spectrum for WAS and WFS is, respectively:

$$
\begin{aligned}
& b_{A}=N b_{a}=\frac{1}{1+\frac{n_{r} s}{N S \frac{\eta_{f}}{\eta_{a}}}}, \\
& b_{F}=\frac{n_{r}}{S} s b_{f}=\frac{1}{1+\frac{N S \frac{\eta_{f}}{\eta_{a}}}{n_{r} s}} .
\end{aligned}
$$

\section{Observations:}

1. As defined, $b_{A}+b_{F}=1$ (normalized)

2. For $n_{r}=0$, (no repeaters): $b_{A}=1, b_{F}=0$; i.e., all resources are used for WAS.

3. In the example of a case where $n_{r}=25$ (access radius 5 times smaller than the feeder radius), $S$ $=6, s=2, N=3, \eta_{f} / \eta_{a}=1.5$, the resulting resource allocation is $b_{A}=0.35, b_{F}=0.65$. Only about one third of radio resources is used for access, the rest for feeder.

If in equation (2) we do not consider a normalized approach, and instead define:

- $\Lambda=$ the total available licensed radio resource, (frequency $\mathrm{x}$ time)

- $C=$ the aggregate throughput to be provided within service area of each FRS

(i.e., in (2) we shall have B on the right side instead of unity)

We can rewrite (5) as

$$
\Lambda=b_{A} \cdot\left(1+\frac{n_{r} s}{N S \frac{\eta_{f}}{\eta_{a}}}\right)=b_{a}\left(N+n_{r} \frac{s}{S \frac{\eta_{f}}{\eta_{a}}}\right)=\frac{C}{\eta_{a}}\left(N+n_{r} \frac{s}{S \frac{\eta_{f}}{\eta_{a}}}\right),
$$

where we have used 


$$
b_{a}=\frac{b_{A}}{N}=\frac{C}{\eta_{a}}
$$

The expressions (7) show that the total required radio resources $\Lambda$ (entire radio spectrum license) grows linearly with $n_{r}$, the number of FRS in every BS (or sector of BS, if BS is sectorized). In the same time, as shown in Figure 2, the amount of the radio resources allocated for access to all FRSs, $b_{A}$, remains constant, since the cluster size $N$ does not change.

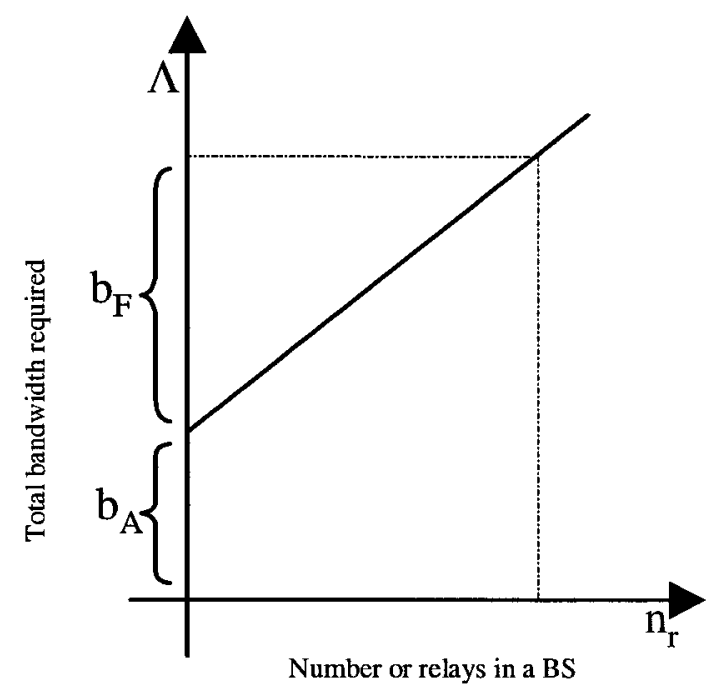

Figure 2. Required license versus the number of FRS in each BS

When the number $n_{r}$ of FRSs in a cell increases to large values (tens), the most part of available resources are allocated to the feeder system, while the access system is allocated only a small proportion of the total system resources, as plotted in Figure 3 (using the same assumptions as in Observation 3 before). Given that this small fraction still has to be sufficient for the high access data rates envisioned, the result is a large increase in total resources required for the entire system (again most of which is used for feeder). On the other side, if the number of relays is too small, there will be gaps in the coverage, where communication at the required data rate is not possible. A larger number of relays means a smaller coverage area for each relay, leading to better average SNR for the access link, which can be exploited through higher order modulation and coding to obtain a better spectral efficiency for the WAS. Increasing the number of relays 
beyond the level at which all coverage gaps are eliminated, (which depends on the system parameters used) will not be accompanied by an important increase in the spectral efficiency for the WAS, not fully compensating for the bandwidth "lost" to additional allocations to the feeder system. So, increasing the number of relays beyond a certain point is inefficient. This is plotted ${ }^{2}$ in Figure 3, where the average net spectral efficiency (defined as the ratio between the access throughput and the total system bandwidth for access and feeder) at the edges of the relay coverage areas is shown for BS radius $R=800 \mathrm{~m}$ (blue) and $R=500 \mathrm{~m}$ (green). We can see that for a smaller BS radius, the number of relays required to fully cover the entire service area is reduced, and the achieved spectral efficiency is higher. Off course, these advantages do not come without costs: an extra $156 \%$ additional base stations with $R=500 \mathrm{~m}$ are needed to cover the same area covered by base stations with $R=800 \mathrm{~m}$.

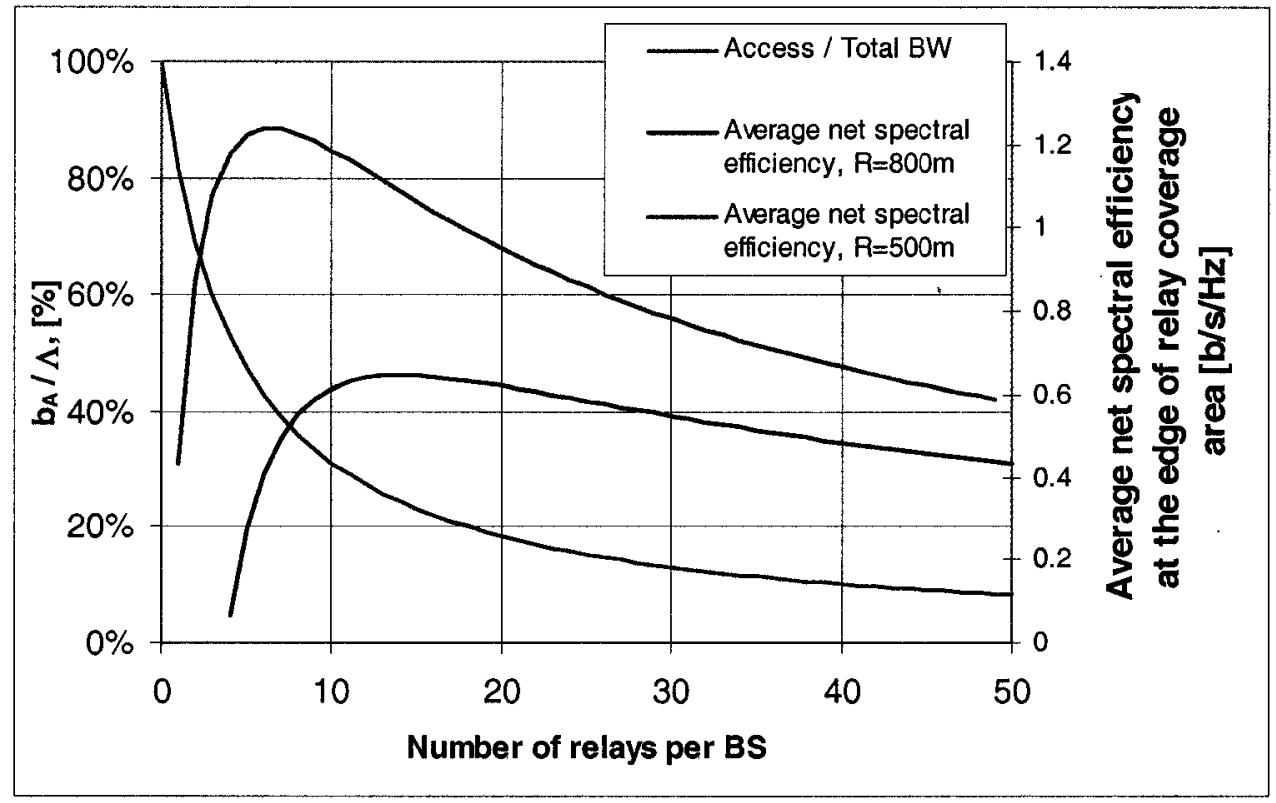

Figure 3. Bandwidth allocated for access vs. number of relays in a BS, $\boldsymbol{n}_{r}$

${ }^{2}$ The system parameters used to plot the average net spectral efficiency are the same as in section 3.4 , with the exception of the path loss exponent $p=4$. The plots are based on Spectral efficiency vs SNR curve presented in Figure 5 and SNR vs. Distance curve presented in Figure 6. 


\subsubsection{A Different View Of The Same Problem}

A consequence of the argument above is that, for example, in order to build a system with a maximum throughput per user $C$ (i.e., a user at peak throughput uses all available bandwidth of the serving FRS; $C$ is assumed $100 \mathrm{Mb} / \mathrm{s}$ in [14]), the feeder system has to be able to accommodate a much larger throughput, by a factor proportional with the ratio of spectral efficiencies for the two systems and the ratio $n_{r} / N$. The BS aggregate throughput is then the maximum throughput offered by the feeder system, exceeding $C(100 \mathrm{Mb} / \mathrm{s})$ many times.

However, in [14] the aggregate BS throughput requirement is assumed to have the same value as the maximum throughput per user, i.e., $100 \mathrm{Mb} / \mathrm{s}$. Under this assumption, if one user requests and is granted the highest possible throughput $C$, then no other users within the BS area will be offered any services. This requirement is less difficult to achieve, since in this case the feeder system has only to accommodate aggregate data rates up to $C$. In the same time, each FRS must be provisioned on a permanent basis with enough radio resources to handle access rates up to the required $C$. With only one user accessing the system at $C=100 \mathrm{Mb} / \mathrm{s}$, all FRS except the one providing service to the sole user will be idle (because the feeder system is fully loaded), which is a tremendous waste of radio resources. The "wasted" amount is again proportional with the ratio $n_{r} / N$.

In conclusion, for an efficient network deployment making the best use of the available limited radio resources (frequency license), the number of relays employed should be kept to a minimum, while still ensuring good coverage of the service area. This suggests that advanced methods (for example MIMO systems, relay cooperation schemes, etc) need to be utilized at the physical layer in order to increase the coverage range of the wireless access system, and reduce the number of relays.

Although the argument above is based on the assumption of a feeder system based on singlehop feeder links, the result can immediately be extended to the case of multi-hop feeder links. To do this, we consider $b_{A}$ being actually the bandwidth needed by only the first hop adjacent to the 
BS, for all multi-hop links to all relays, and we can apply directly the same argument as before. In the case of multi-hop feeder links, the total bandwidth allocated to the feeder system is actually equal or larger than $b_{A}$, leading to a potentially even more inefficient deployment scenario.

In the following sections we analyze the multi-hop links forming feeder systems and identify deployment strategies minimizing the total bandwidth required by the feeder system.

\subsection{Multi-hop Link Analysis}

We consider the multi-hop link $\mathrm{R}_{0}-\mathrm{R}_{\mathrm{n}}$ in the feeder part of a fixed relay network with an $n$-hop link, as shown in Figure 4, where $R_{0}$ and $R_{n}$ are the source and the recipient stations, respectively. The message can be either sent directly from $R_{0}$ to $R_{n}$ (single-hop operation), or the message can be sent via $n$ - 1 intermediate fixed relays over $n$ hops.

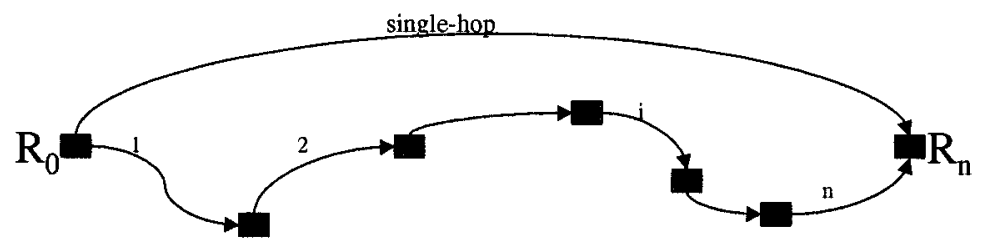

Figure 4. Multi-hop link topology.

The relays are of the digital regenerative type. Each relay is using only the information received from its immediate predecessor in the chain, i.e. there is no diversity combining of received signals from multiple "uphill" transmitters. Since the relays are fixed, the topology of the system is considered known and non-dynamic, so no routing algorithm is required. As the overhead messaging related to multi-hop functionality will not be significant, it was not considered as a factor in the discussion below. As well, the processing delays in the relays have been considered to be much smaller compared with the transmission time of relayed data packets.

The system uses a time-slotted resource allocation scheme, where each radio link is assigned a channel in the frequency - time domain. Each intermediate radio link adopts an appropriate 
modulation scheme resulting in the best possible spectral efficiency based on the given SNR conditions.

We adopt the following assumptions as stated in [17]:

1. The amounts of data received and transmitted by each relay are equal.

2. For simplicity we assume that all $n$ hops of the multi-hop link, and as well the single-hop $\mathrm{R}_{0}-\mathrm{R}_{\mathrm{n}}$ are allocated the same amount of bandwidth $B$ accessed in a time-division manner. The individual time required to pass a message over the hop $i$ is

$$
t_{i}=\frac{M}{B \eta_{i}}
$$

where $M$ is the message size in bits and $\eta_{i}$ is the spectral efficiency (in bits/sec/Hz) of the radio link over hop $i$.

3. The timeslots $t_{i}$ allocated to each hop in the multi-hop link are considered orthogonal to each other. Also for simplicity but without loss of generality, we assume that all links operate on the same carrier frequency. Although the orthogonality condition seems conservative, as it will be seen later, in most cases the number of hops envisioned for the multi-hop link is three or less; with such a low number of intermediate links and given the high SNR required by the desired data rates, it is unlikely that any frequency or time slot reuse would be possible, unless advanced processing techniques or antenna architectures are employed. In conclusion, the total time, $T$, required to pass a message of size $M$ from $\mathrm{R}_{0}$ to $\mathrm{R}_{\mathrm{n}}$ is the sum of all intermediate timeslots:

$$
T=\sum_{i=1}^{n} t_{i}=\frac{M}{B} \sum_{i=1}^{n} \frac{1}{\eta_{i}}
$$

4. Since the channels used by each hop in the multi-hop link are orthogonal, no co-channel interference is present.

5. All radio links in the system have similar radio propagation parameters. 


\subsection{Optimal Relay Locations}

We observe that the spectral efficiency for a given link has an approximate linear dependency on the SNR measured in $\mathrm{dB}$, as shown in Figure $5 .{ }^{3}$

We can approximate the spectral efficiency for a single hop, $\eta$, and for the hop $i$ of the multihop link, $\eta_{i}$ as

$$
\begin{aligned}
& \eta \cong K_{1} \log _{10} \gamma, \\
& \eta_{i} \cong K_{1} \log _{10} \gamma_{i},
\end{aligned}
$$

where $\gamma$ and $\gamma_{i}$ are the SNR for the direct link $\mathrm{R}_{0}-\mathrm{R}_{\mathrm{n}}$ and link $i$, respectively, and $K_{1}$ is a constant of proportionality. The expressions (11) and (12) remain valid for other digital modulation schemes as long as the linear dependency as in Figure 5 is preserved (straight line passing through origin).

Although the expressions (11) and (12) do resemble the Shannon capacity formula

$$
C[\text { bits } / s / H z]=\log _{2}(1+\gamma) \cong 3.32 \log _{10}(1+\gamma),
$$

they are not identical. However, it can be observed in Figure 5 that for high SNR values, (more than $15 \mathrm{~dB}$ ), the Shannon values are also following a similar progression law as in (11), albeit with a higher slope than the line of the practical spectral efficiency values.

Assuming that all transceivers at $\mathrm{R}_{0}, \mathrm{R}_{\mathrm{n}}$, and the $n-1$ fixed relays are identical (in terms of transmit power, transmit and receive antenna gains, and receiver noise figure), we can express the mean SNR (excluding shadowing) for the single hop and link $i$ in the $n$-hop case as

$$
\begin{aligned}
& \gamma=K_{2}\left(\frac{D}{d_{0}}\right)^{-p} \\
& \gamma_{i}=K_{2}\left(\frac{d_{i}}{d_{0}}\right)^{-p}
\end{aligned}
$$

\footnotetext{
3 The spectral efficiency figures used to generate the plot in Figure 5 assume Bit Interleaved Coded Modulation [18]. The data were provided by Dr. Sirikiat Lek Ariyavisitakul.
} 


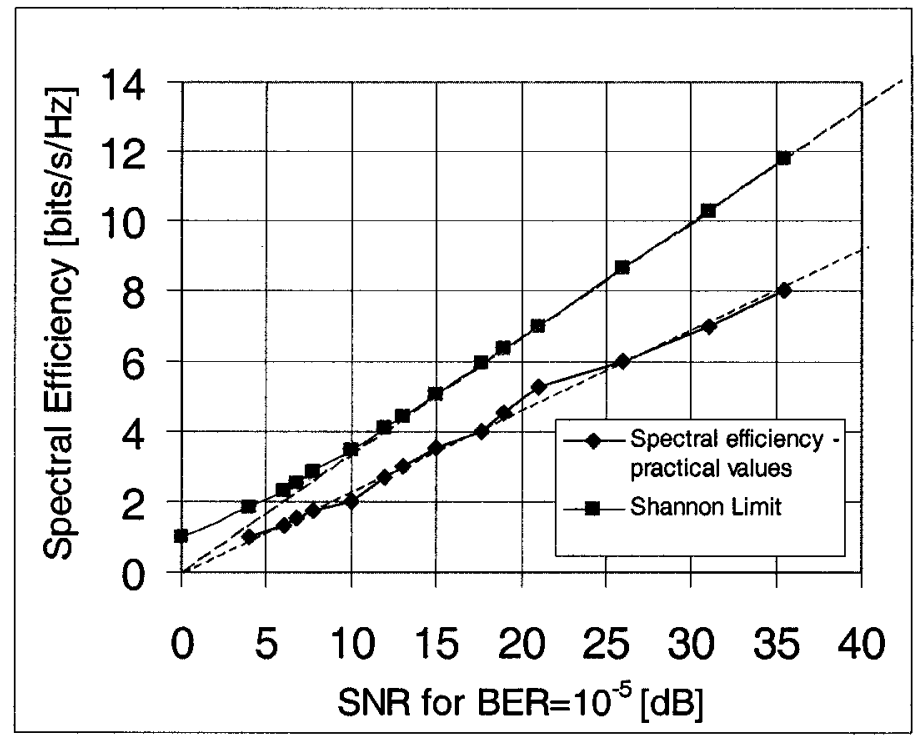

Figure 5. Spectral Efficiency vs. SNR.

respectively, where:

- $\quad D=$ distance $\mathrm{R}_{0}-\mathrm{R}_{\mathrm{n}}$

- $\quad d_{\mathrm{i}}=$ the length of the hop $i$

- $\quad d_{0}=$ the reference close-in distance in radio propagation

- $K_{2}=$ a constant of proportionality

- $\quad p=$ the path loss exponent

In general the constant $K_{2}$ captures the radio link system gains, or the link costs in terms of radio resources:

$$
K_{2}=\frac{P_{T} G_{T} G_{R} \lambda^{2}}{\left(4 \pi d_{0}\right)^{2} P_{N}}
$$

In (15), $P_{T}$ is the transmit power; $G_{T}$ and $G_{R}$ are transmit and receive antenna gains; $P_{N}$ is the noise power; $\lambda$ is the radio wavelength, and $d_{0}$ was introduced earlier as the reference close in distance for radio propagation.

The total time required to pass the message over the multi-hop link can then be expressed as 


$$
\begin{aligned}
& T=\frac{M}{B K_{1}} \sum_{i=1}^{n} \frac{1}{\log _{10} \gamma_{i}} \\
& =\frac{M}{B K_{1}} \sum_{i=1}^{n} \frac{1}{\log _{10}\left(K_{2}\left(\frac{d_{i}}{d_{0}}\right)^{-p}\right)}
\end{aligned}
$$

Our goal is to maximize the end-to-end spectral efficiency and minimize the bandwidth needed for transmitting the message from source to destination ${ }^{4}$. Improving the aggregate end-toend spectral efficiency means reducing the message transfer time. To minimize the sum in (16) we consider the function

$$
f\left(d_{i}\right)=\frac{1}{\log _{10}\left(K_{2}\left(\frac{d_{i}}{d_{0}}\right)^{-p}\right)}
$$

It can be shown that the function $f\left(d_{i}\right)$ is strictly convex for the interval

$$
\frac{K_{2}^{\frac{1}{p}}}{e^{2}}<\frac{d_{i}}{d_{0}}<K_{2}^{\frac{1}{p}}
$$

and strictly concave for

$$
\frac{d_{i}}{d_{0}}<\frac{K_{2}^{\frac{1}{p}}}{e^{2}}
$$

for $i=1,2, \ldots, n$. The convexity and concavity intervals in (18) and (19) can also be expressed in SNR terms as:

$$
\begin{aligned}
& 1<\gamma_{i}<e^{2 p} \\
& \gamma_{i}>e^{2 p}
\end{aligned}
$$

${ }^{4}$ In other studies, different minimization targets may be proposed; for example, unlike our approach, in sensor networks the goal is to minimize the power transmitted by the network elements. 
respectively. It is to be noted that the model adopted works only for SNR values larger than unity, (i.e., $\frac{d_{i}}{d_{0}}>K_{2}^{\frac{1}{p}}$ ), in which case the spectral efficiency and the corresponding message transfer time remain positive.

For the interval where $f\left(d_{i}\right)$ is strictly convex, applying Jensen's inequality [19], we obtain

$$
\sum_{i=1}^{n} \frac{1}{\log _{10}\left(K_{2}\left(\frac{d_{i}}{d_{0}}\right)^{-p}\right)} \geq \frac{n}{\log _{10}\left(K_{2}\left(\frac{\sum_{i=1}^{n} d_{i}}{n d_{0}}\right)^{-p}\right)}
$$

with equality if

$$
d_{1}=d_{2}=\ldots=d_{n} .
$$

On the other side, if $f\left(d_{i}\right)$ is strictly concave, then

$$
\sum_{i=1}^{n} \frac{1}{\log _{10}\left(K_{2}\left(\frac{d_{i}}{d_{0}}\right)^{-p}\right)} \leq \frac{n}{\log _{10}\left(K_{2}\left(\frac{\sum_{i=1}^{n} d_{i}}{n d_{0}}\right)^{-p}\right)}
$$

also with equality if (23) is true,.

We consider the special case of a $n$-hop link with "short" intermediate links, i.e., all intermediate hops lengths $d_{i}$ respect the condition (19), and as a consequence all intermediate hop SNRs $\gamma_{i}$ are as in (21), and thus $f\left(d_{i}\right)$ is strictly concave. According with (24), such a $n$-hop link would have a better aggregate spectral efficiency (smaller message transfer time) compared to a $n$-hop link having the length of all intermediate hops the same and equal to the mean hop length $\bar{d}_{i}=\sum_{i=1}^{n} d_{i} / n$.

Further, in the particular case when all relays are placed on the straight line $R_{0} R_{n}$, any configuration has a better aggregate spectral efficiency compared with the situation when the $n-1$ relays are distributed along equal intervals on the line $\mathbf{R}_{0} R_{n}$. In other words, if all relays are placed on the straight line $\mathrm{R}_{0} \mathrm{R}_{\mathrm{n}}$, evenly spaced relays achieve the worst performance (lower bound) in terms of spectral efficiency. 
We consider next the opposite case of a $n$-hop link with "long" intermediate links, we assume that all hops lengths $d_{i}$ respect the condition (18), therefore the intermediate SNRs $\gamma_{i}$ are as in (20), and in return $f\left(d_{i}\right)$ is strictly convex. In this case, (22) stands true.

Geometrically, the smallest value of the sum of all $n$ individual hop lengths is reached when all relays are placed on the straight line $\mathrm{R}_{0} \mathrm{R}_{\mathrm{n}}$. In that case

$$
\sum_{i=1}^{n} d_{i}=D
$$

As a result, the best possible geographical locations for the $n-1$ intermediate fixed relays which would minimize the sum term in (16) and the total message transfer time $T$ are along equal intervals on the straight line $\mathrm{R}_{0} \mathrm{R}_{\mathrm{n}}$. In such a case,

$$
d_{i}=\frac{D}{n} \quad \text { for } i=1,2, \ldots, n .
$$

We have reached the apparently peculiar conclusion that there is no unique optimal configuration for the locations of the $n$ - 1 relays. For "long" intermediate hops, the configuration with evenly distributed relays is optimal, while for "short" intermediate hops, it is the worst. This can be explained by taking a close look at the underlying mathematical model assumed for the spectral efficiency and the message transfer time.

From equations (12) and (14) we can see that as the hop length grows, the SNR value in $\mathrm{dB}$ decreases linearly, while the message transfer time (MTT) increases exponentially, as shown in the example in Figure 6. The variation of MTT with distance is more accentuated for large hop lengths, and less accentuated at shorter distances. Thus, assuming that all intermediate hop lengths are short, changing the hop lengths will not have a great impact on the total MTT. We could even imagine setting the length of one of the intermediate hops to zero (which is equivalent with removing the hop altogether), and distributing its length to the remaining ones; if the added length will not bring any of the remaining hops within the category "long", the net effect will be to reduce the total aggregate MTT, since now there is one less hop, while the remaining hops have only slightly larger individual MTTs. Continuing this argument, and 
assuming that the "short" (i.e., the concavity) condition holds, we can repeat the same process until there is a single hop, every time reducing the aggregate MTT.

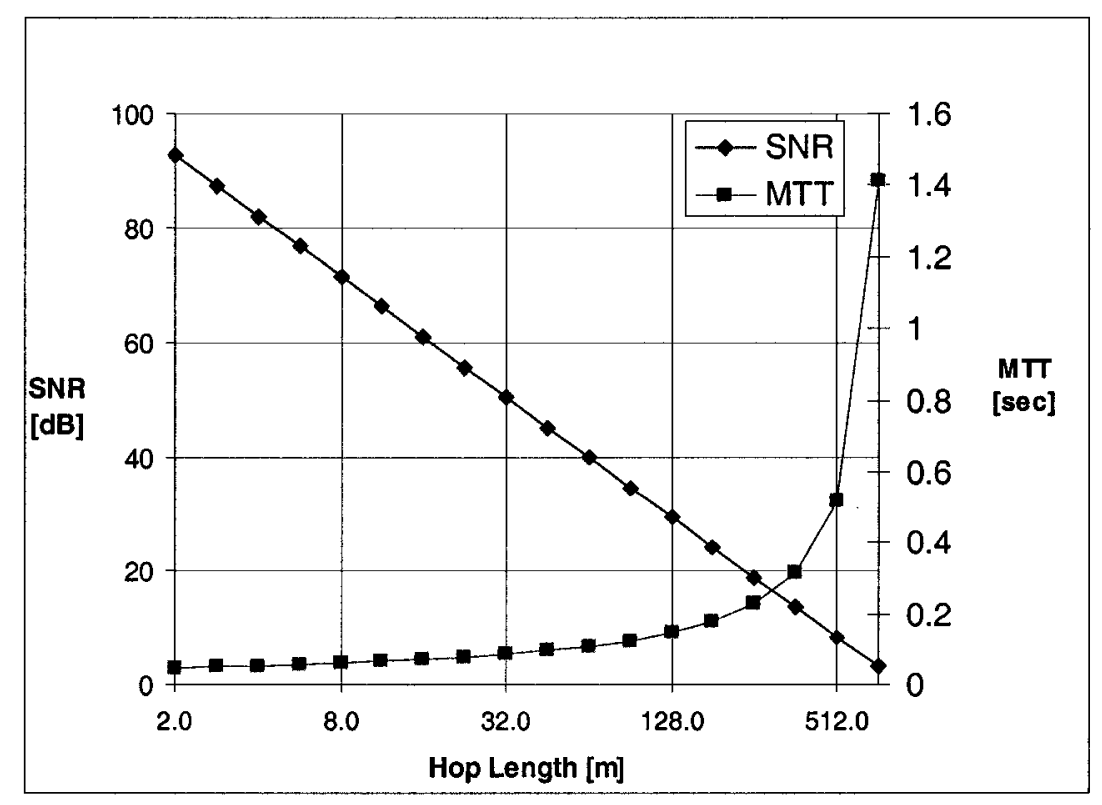

Figure 6. Message Transfer Time vs. Hop Length

For such a multi-hop link, the optimal locations for all relays is at either the source or at the destination locations. In other words, if the concavity condition holds, the single-hop link will always outperform the multi-hop link.

For the "long" intermediate hops, the situation is the opposite: MTM has a very strong dependency on the hop-length, and the slope of the curve is sharper and sharper at higher values . Within the condition that all hops are "long" (convexity condition), it makes sense to redistribute the length by shortening the longest hops; the losses in MTT from shortening longer hops more than compensates for increases in MTT in shorter ones, due to the slope difference, and the aggregate MTT is reduced. At the limit, the optimal relay locations which minimize aggregate MTT is the one which minimized all hop lengths, i.e., at equal intervals on the straight line $R_{0} R_{2}$.

To graphically show this behavior, we compare the MTT for a two-hop link having the relay placed at an arbitrary position, with the MTT of the same two-hop with the relay located at equal 
distances from source and destination. It would be unfair to compare two-hop links with unequal sums of the intermediate hop lengths; the longer two-hop link would have necessarily a larger MTT. Thus, we compare (take the ratio between) MTT of a two-hop of lengths $\left(d_{1}, d_{2}\right)$ and the MTT for a hypothetical comparison link with hop lengths $\left(\left(d_{1}+d_{2}\right) / 2,\left(d_{1}+d_{2}\right) / 2\right)$. In other words, the comparison relay location is on the same ellipse (of focal centers $R_{0}$ and $R_{2}$ ) at equal distance from $R_{0}$ and $R_{2}$, see Figure 7 . The value of the ratio - below or above unity - indicates in which cases the "relay in the middle" scenario is optimal.

If any of the intermediate hops has a length larger than the original single hop, the multi-hop MTT will be necessarily higher compared with the single-hop. Thus, relay's geometric possible locations respect $d_{1}, d_{2} \leq D$ as shown in Figure 7, being within the intersection of two circles of radius $D$ and centers $R_{0}$ and $R_{2}$, respectively.

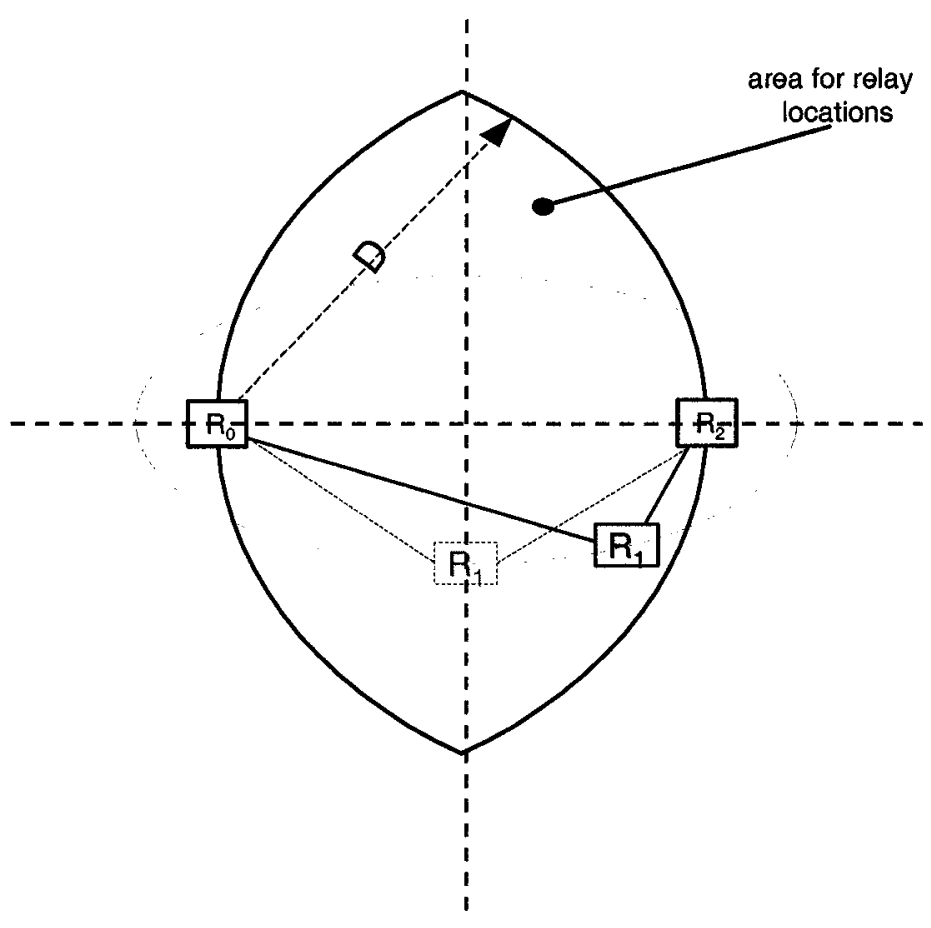

Figure 7. Geometric possible locations for relays in a two-hop link

Assuming the system parameters $P_{T}=30 \mathrm{dBm}, G_{T}=10 \mathrm{~dB}, G_{R}=10 \mathrm{~dB}, P_{n}=-95 \mathrm{dBm}, d_{0}=$ $2 m, p=3.5, \lambda=0.06 m$, an intermediate hop is "short" if 


$$
d_{i}<d_{0} \frac{K_{2}^{\frac{1}{p}}}{e^{2}} \approx 119.6 m
$$

In Figure 8, for graphical reasons we have plotted the time ratio against the changed and normalized variables $\left(d_{1}+d_{2}\right) / D-1,\left(d_{1}-d_{2}\right) / D$. This ensures that the geometric locations of the straight line $\mathrm{R}_{0} \mathrm{R}_{2}$ and as well the locations where $d_{l}=d_{2}$ are easy to identify, as shown on the first plot, in Figure 8, a). Various surfaces are plotted for different values of the distance $R_{0} R_{2}$ $D$, which determines the 2-hop link $\mathrm{R}_{0} \mathrm{R}_{2}$ to have "short" intermediate hops or not. For distances $\mathrm{R}_{0} \mathrm{R}_{2}$ smaller than the value in (27) of about $120 \mathrm{~m}$, both intermediate hops are always "short" and the message transfer time is largest when the relay is placed in the middle of the segment $R_{0} R_{2}$. We can see from the plots that the best relay location in this case is as close as possible to either $\mathrm{R}_{0}$ or $\mathrm{R}_{2}$, which seems to suggest that for "short" intermediate hops the single-hop link outperforms the 2-hop link (within the framework of the initial assumptions, the 2-hop link with the relay placed at one end is equivalent with a single-hop link). For larger distances $R_{0} R_{2}$, for example $D=220 \mathrm{~m}$, the situation changes as follows: if the relay is located close to the straight line $\mathrm{R}_{0} \mathrm{R}_{2}$ (where $d_{1}+d_{2}=D$ ), the two hop lengths are "short", and the best relay locations are close to $R_{0}$ or $R_{2}$; however for relays located farther of the $R_{0} R_{2}$ line, the best relay location is at equal distances from $R_{0}$ and $R_{2}$. For even larger distances $R_{0} R_{2}$, the middle of the segment $R_{0} R_{2}$ is optimal location for the relay (with the exceptions of locations very close to either $R_{0}$ or $R_{2}$ ), and the use of the relay improves the overall performance compared with the single-hop.

To find out the threshold value when the 2-hop link becomes more efficient than the singlehop $\mathrm{R}_{0} \mathrm{R}_{2}$, we set the condition that the message transfer time is the same for the single-hop and for the 2-hop link with the relay placed in the middle of the segment $R_{0} R_{2}$ : 


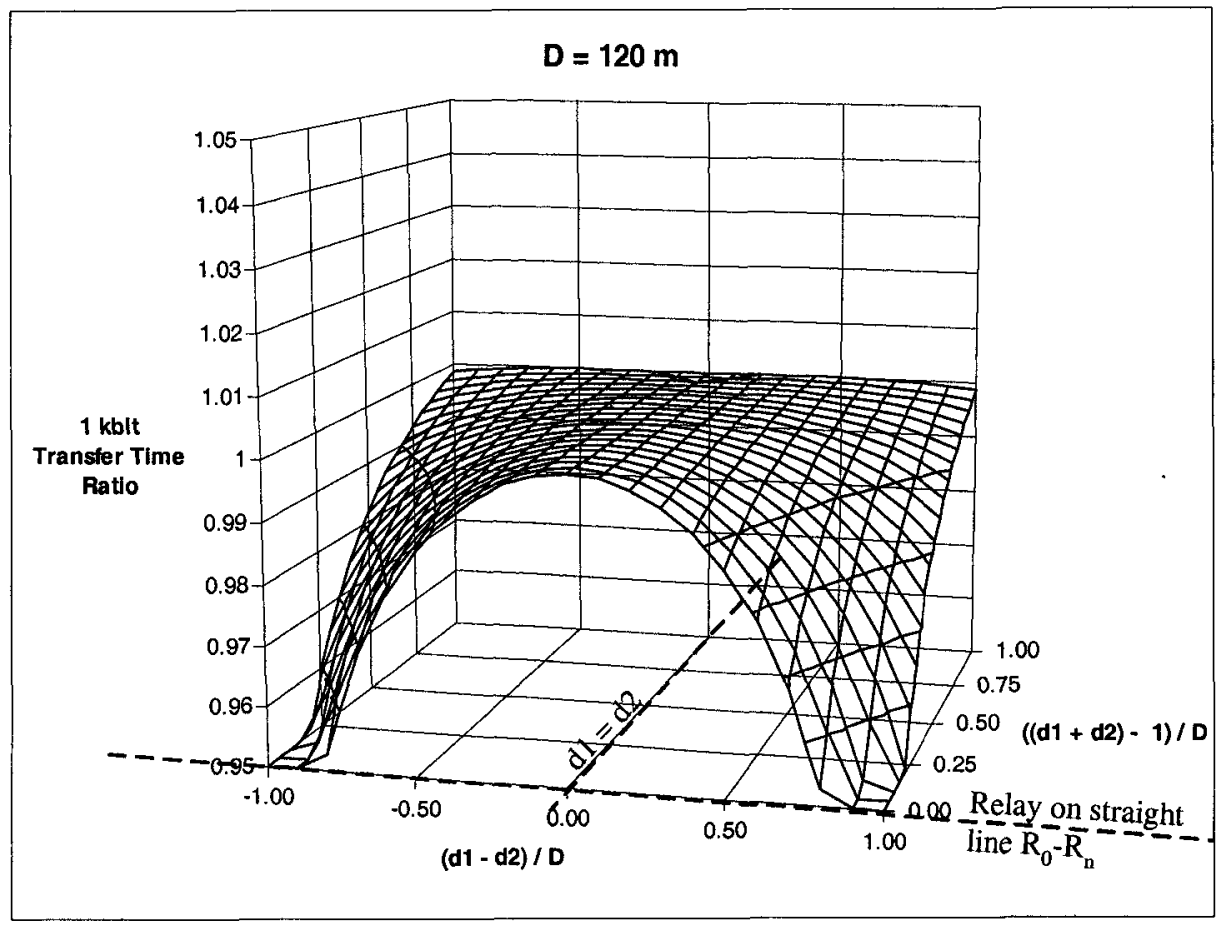

a)

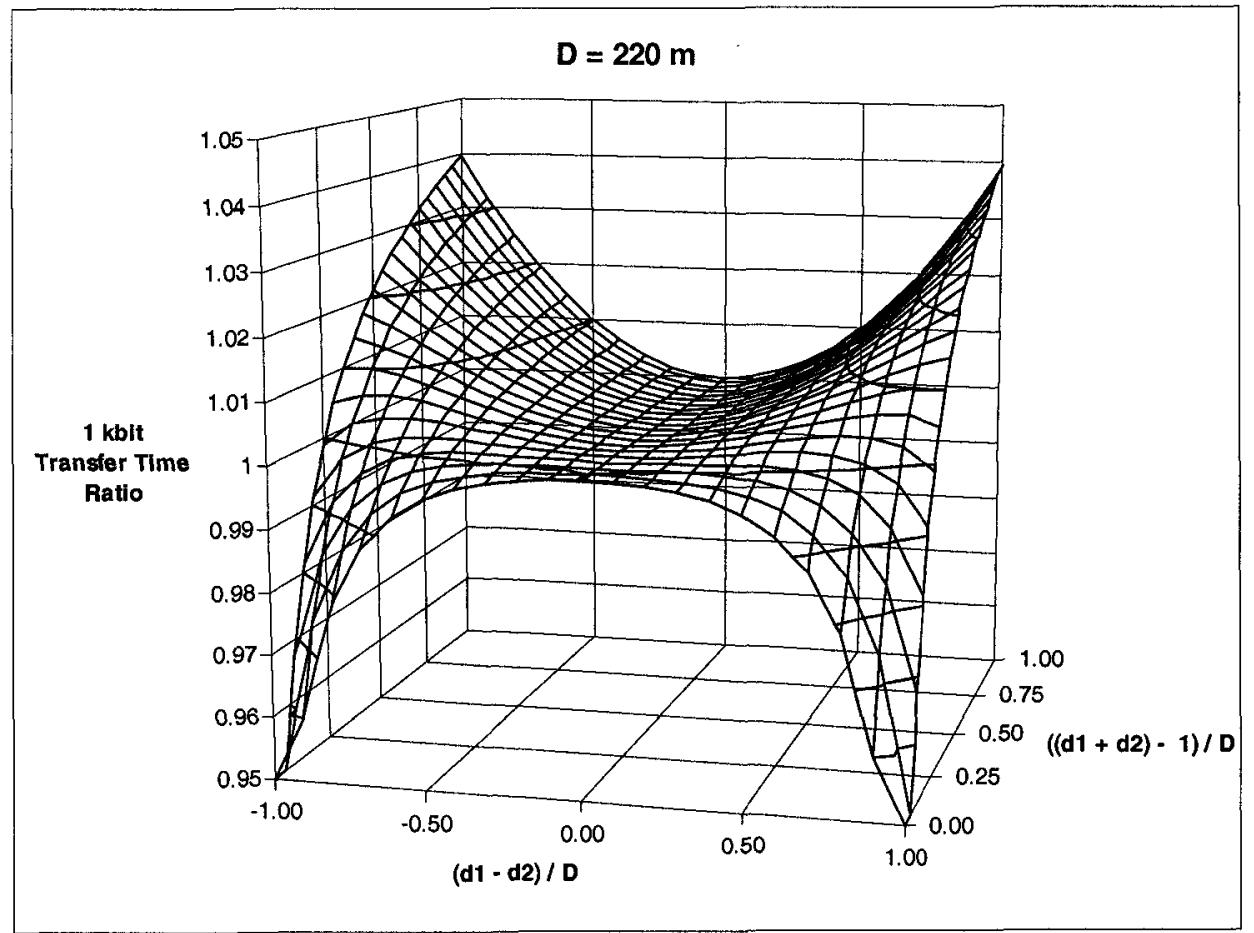

b) 


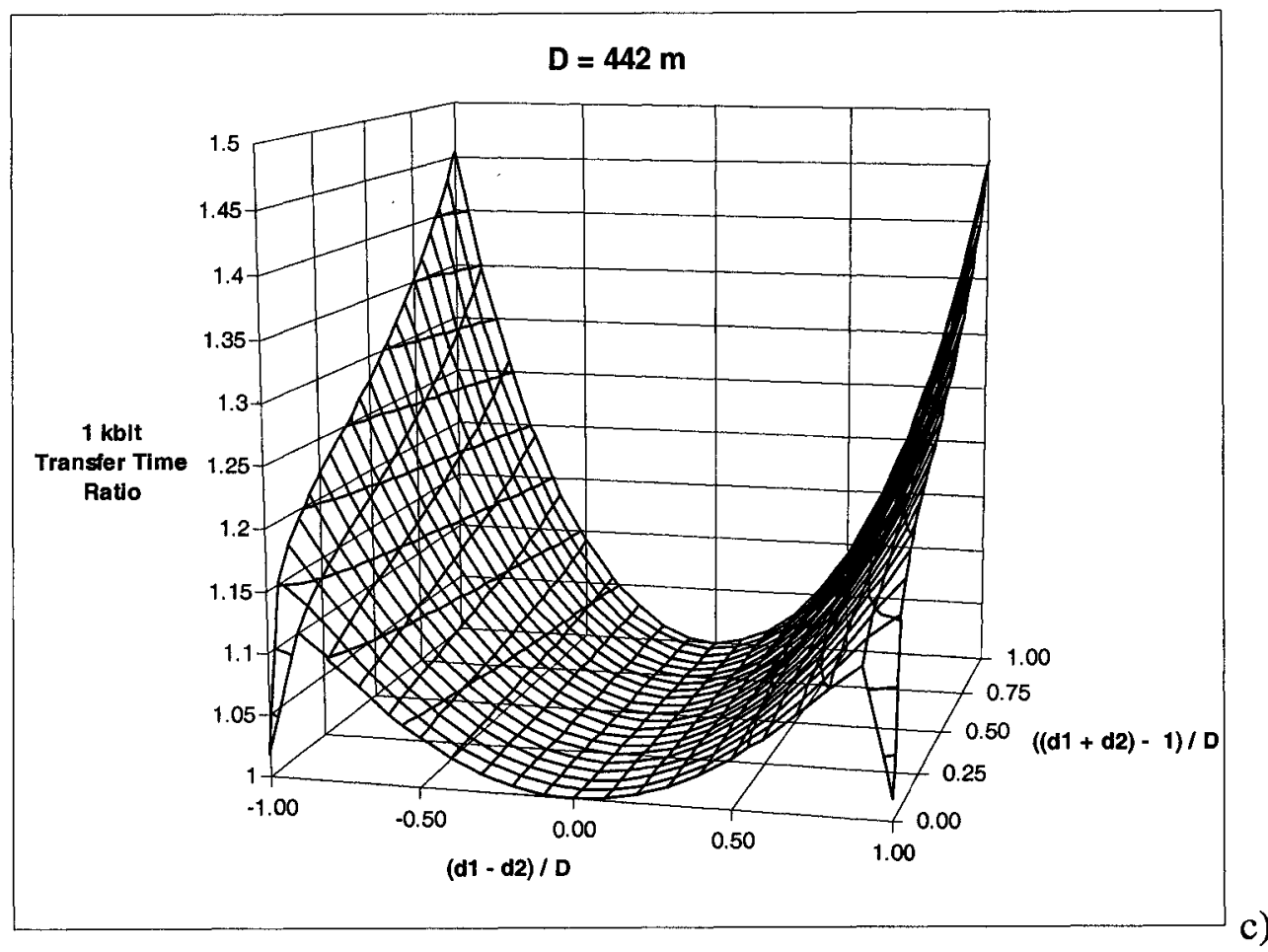

Figure 8 a), b), c). The ratio between message transfer times of a two-hop link and the link with the two hops of length $\bar{d}_{i}$.

$$
\frac{1}{\log _{10}\left(K_{2}\left(\frac{D}{d_{0}}\right)^{-p}\right)}=\frac{2}{\log _{10}\left(K_{2}\left(\frac{D}{2 d_{0}}\right)^{-p}\right)}
$$

Simplifying (28) we obtain

$$
\frac{D}{d_{0}}=\frac{1}{2} K_{2}^{\frac{1}{p}} \text {, }
$$

or, $D=442 m$ for the same systems parameters as before. As seen in Figure $8 \mathrm{c}$ ), for $\mathrm{R}_{0} \mathrm{R}_{2}$ distances $D=442 m$ and larger, the optimal relay location is the middle of the segment $R_{0} R_{2}$. In SNR terms (29) can be expressed as:

$$
\gamma=2^{p}
$$

We can conclude that, for the link $\mathrm{R}_{0} \mathrm{R}_{2}$, if the single hop SNR is larger than $2^{p}$, any 2-hop link would have a lower performance than the single hop. If the signal to noise ratio is below $2^{p}$, 
a better aggregate spectral efficiency is achieved by the 2-hop link with the relay placed in the middle of $R_{0} R_{2}$.

\subsection{Multi-Hop Criterion}

It is more advantageous to use the $n$-hop link if the end-to-end spectral efficiency is improved. With the message size $M$ and bandwidth $B$ being the same, a single-hop link should better be replaced by a multi-hop link if

$$
\sum_{i=1}^{n} \frac{1}{\eta_{i}}<\frac{1}{\eta}
$$

where $\eta$ represents the spectral efficiency for the single-hop link $\mathrm{R}_{0}-\mathrm{R}_{\mathrm{n}}$. The condition (31) simply states that in order for the multi-hop link to be more efficient, the time required to pass a message of a given size $M$ from $\mathrm{R}_{0}$ to $\mathrm{R}_{\mathrm{n}}$ over the single-hop link must be larger that the time required for the same operation over the multi-hop link.

To generalize the relation (30) to the case of $n$-hop links, using (22-23) and (25), we express the lower bound on the message transfer time using $n$-hops as

$$
\begin{aligned}
& T \geq \frac{M}{B K_{1}} \frac{n}{\log _{10}\left(K_{2}\left[\frac{D}{n d_{0}}\right]^{-p}\right)} \\
& =\frac{M}{B K_{1}} \frac{n}{\log _{10}\left(n^{p} \gamma\right)} .
\end{aligned}
$$

The expression (32) shows the smallest possible message transfer time using $n$-hops, given that $(20)$ is true. If the single-hop link can transfer the data in a shorter or equal time, then there would be no point in using relays. Using (32), the inequality (31) can be rewritten as

$$
\frac{n}{\log _{10}\left(n^{p} \gamma\right)}<\frac{1}{\log _{10} \gamma}
$$

which can be simplified to the expression

$$
\gamma<n^{\frac{p}{n-1}}
$$


If for a given single-hop link condition (34) is not met, a $n$-hop link replacement with a better overall spectral efficiency does not exist, no matter where the relays are located. The inequality (34) represents a quantitative criterion which can be used to decide in which situation a multihop link could be used.

The "break-even" SNR values in (34) are plotted in Figure 9 for various values of the path loss exponent $p$.

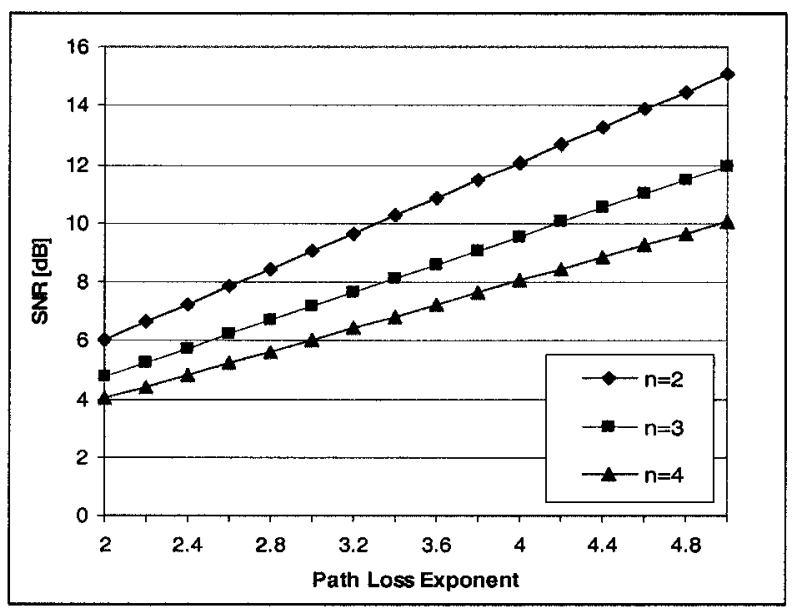

Figure 9. SNR values above which the single-hop has better spectral efficiency compared to any $n$-hop

The plots show the variation of the threshold (or "break-even") SNR value at which a more efficient multi-hop alternative to the given single-hop link becomes feasible. For example, in Figure 9, if, for instance, the path loss exponent of all intermediate hops happens to be $p=3.6$, then, according to (34), there exists a possible 3-hop configuration (the 2 relays evenly distributed along the line $\mathrm{R}_{0} \mathrm{R}_{\mathrm{n}}$ ) with a better aggregate spectral efficiency, as long as the single-hop link SNR is less than $8.6 \mathrm{~dB}$.

\section{Remarks:}

1. The criterion developed above uses as a performance metric the bandwidth (frequency-time) needed for transferring messages from source to destination. The energy required for transfer is not considered as a metric; that is, the additional power inserted in the system by intermediate relays is considered "free". 
2. As the discussion above is based on mean SNR values not including shadowing, these results are only applicable for a statistical average over a set of multi-hop links; the results above are not binding on a given particular realization of a multi-hop link.

3. As expected, in the case of $n=2$ (one fixed relay between $R_{0}$ and $R_{n}$ ), the inequality (34) becomes $\gamma<2^{p}$, in concordance with (30). For example, if the propagation exponent has a value of 3 , one relay placed in the ideal location (right in the middle of the link $R_{0}-R_{n}$ ) would be efficient only if the SNR of the link $R_{0^{-}}-R_{n}$ is less than $8(9 \mathrm{~dB})$.

4. The multi-hop criterion is in general applicable to multi-hop links with all individual hops having similar radio propagation characteristics, i.e. equal path loss exponents. In the special case when the mobile access link (the last hop of a $4 \mathrm{G}$ cellular link using fixed relays) has the same path loss exponent as the feeder system, the multi-hop criterion can be extended to cover the entire link between the base station and the mobile terminal.

\subsubsection{Reduced path loss exponent for shorter links}

In practice, the likelihood that a longer radio path will be obstructed by some obstacle(s) is larger than the probability of obstruction for a shorter path. Since the intermediate multi-hop links are considerably shorter than the single-hop $\mathrm{R}_{0}-\mathrm{R}_{\mathrm{n}}$, they are likely subject to different propagation characteristics compared with the single-hop $\mathrm{R}_{0}-\mathrm{R}_{\mathrm{n}}$. With the assumption that the path loss exponents of shorter intermediate hops are all equal, and smaller than the path loss of the longer single-hop $R_{0}-R_{n}$, we have

$$
p_{1}=p_{2}=\ldots=p_{i}=\ldots p_{n}=\alpha p
$$

where $\alpha$ is a real scalar with $2 / p \leq \alpha \leq 1$, and $p_{i}, i=1,2, \ldots, n$, is the path loss exponent for hop $i$. In these conditions equation (31) can be expanded as 


$$
\sum_{i=1}^{n} \frac{1}{\log _{10}\left(K_{2}\left(\frac{d_{i}}{d_{0}}\right)^{-o p}\right)} \leq \frac{1}{\log _{10}\left(K_{2}\left(\frac{D}{d_{0}}\right)^{-p}\right)}
$$

The "short" and "long" hop conditions become in this case $\gamma_{i}>e^{20 p}$, and $1<\gamma_{i}<e^{2 o p}$, respectively. Assuming the "long" hop conditions, the lower bound of the sum in (36) is reached when all relays are located along equal intervals along the straight line $R_{0} R_{n}$. In this situation, using (26) we can rewrite (36) as

$$
\frac{n}{\log _{10}\left(K_{2}\left(\frac{D}{n d_{0}}\right)^{-a p}\right)} \leq \frac{1}{\log _{10}\left(K_{2}\left(\frac{D}{d_{0}}\right)^{-p}\right)}
$$

Simplifying (37) we obtain

$$
K_{2}^{n}\left(\frac{D}{d_{0}}\right)^{-p n} \leq n^{\alpha p} K_{2}\left(\frac{D}{d_{0}}\right)^{-\alpha p}
$$

which can be further compressed considering equation (13):

$$
\gamma \leq K_{2}^{\frac{1-\alpha}{n-\alpha}} \frac{\alpha}{n^{n-\alpha}}
$$

Expression (39) is a more general expression for the Multi-Hop Criterion, here taking into account different values of the path loss exponent for the single-hop link, and for the intermediate hops in the multi-hop link.

We plotted (39) in Figure 10 for $\alpha=0.8$ and $\alpha=0.9$. The system parameters used to calculate parameter $K_{2}$ are the same as assumed in equation (27). Comparing with Figure 9, we observe 
that the "break-even" SNR values when a multi-hop is feasible are higher when the intermediate hops path loss exponent is reduced.
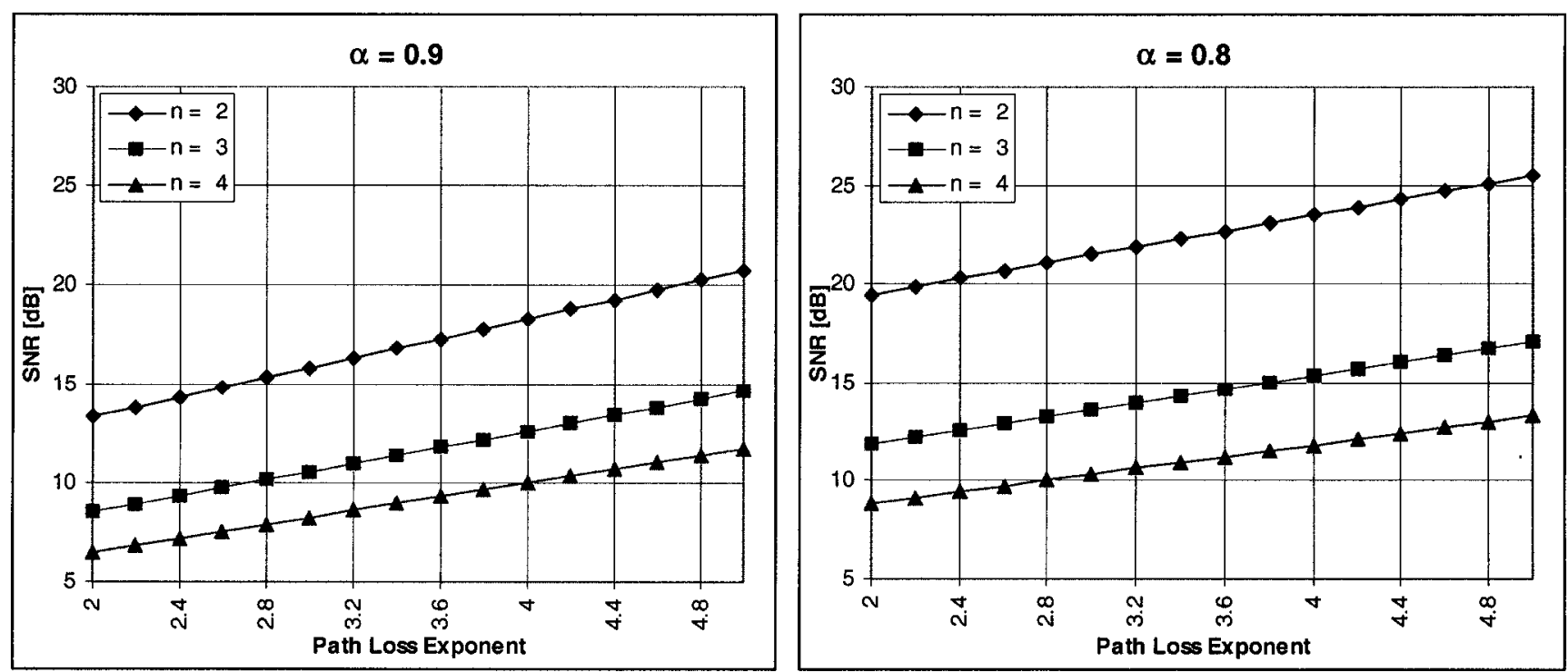

Figure 10. MHC break-even SNR values. Intermediate hops have a

reduced path loss exponent

\section{Remarks:}

1. For $\alpha=1$, i.e., same path loss exponent for all radio links, equation (39) becomes identical with (34).

2. The first term in the right side of the expression (39) shows the dependency of break-even SNR value (when it becomes efficient to split the link into $n$-hops) of the radio link costs. As expected, higher link costs (which translate into a higher value for the $K_{2}$ parameter) lead to an increased SNR break-even value. In other words, if we would attempt to improve the single-hop $R_{0}-R_{n}$ spectral efficiency link by increasing its costs - for example increasing transmit power, antenna gains, etc. - this would not make the single-hop link more efficient than its multi-hop replacement. 


\subsection{System Model Considering Shadowing}

The system model considered in the previous sections is valid for statistical averages over sets of multi-hop links. To evaluate a particular multi-hop link we need to take into account the additional path losses due to local terrain and man-made obstacles. Including log-normal shadowing in the path loss model, the MTT for the multi-hop link can be expressed as

$$
T=\frac{10 M}{B K_{1}} \sum_{i=1}^{n} \frac{1}{10 \log _{10}\left(K_{2}\left(\frac{d_{i}}{d_{0}}\right)^{-p}\right)+X_{i}}
$$

Where $X_{i}, i=1,2, \ldots, n$, are i.i.d. Gaussian random variables, with zero mean and standard deviation $\sigma[21]$.

The denominator of each term under the sum in equation (40) can be itself considered as a Gaussian random variable $Y_{i}$, and we can write

$$
\begin{gathered}
T=\frac{10 M}{B K_{1}} \sum_{i=1}^{n} \frac{1}{Y_{i}} \\
Y_{i} \sim N\left(10 \log _{10}\left(K_{2}\left(\frac{d_{i}}{d_{0}}\right)^{-p}\right), \sigma^{2}\right)
\end{gathered}
$$

To evaluate analytically the sum in (41) we would need to determine the statistical properties of the reciprocal of a Gaussian random variable with known mean and variation. This turns out to be quite a difficult task. The reason is, regardless of the mean and variation values of $X_{i}$, there is always a small probability that $X_{i}=0$, in which case $I / X_{i}$ is not defined.

A closed form for the pdf of the $Y_{i}$ is relatively easy to determine as follows [20]:

$$
\begin{gathered}
F_{Y}(y)=P[Y \leq y]=P\left[\frac{1}{X} \leq y\right]=P\left[X \geq \frac{1}{y}\right] \cong 1-F_{x}\left(\frac{1}{y}\right) \\
f_{Y}(y)=\frac{d}{d y} F_{Y}(y)=\frac{d}{d y}\left[1-F_{X}\left(\frac{1}{y}\right)\right]=f_{X}\left(\frac{1}{y}\right) \frac{1}{y^{2}}=\frac{1}{\sqrt{2 \pi} \sigma_{X} y^{2}} e^{-\frac{\left(1-m_{X} y\right)^{2}}{\sigma_{X}{ }^{2} y^{2}}}
\end{gathered}
$$


The pdf expression determined in (42) verifies with the experimental data (computer generated) as shown in Figure 11, where the pdf is plotted for different values of the mean and variance of the originating Gauss random variable.
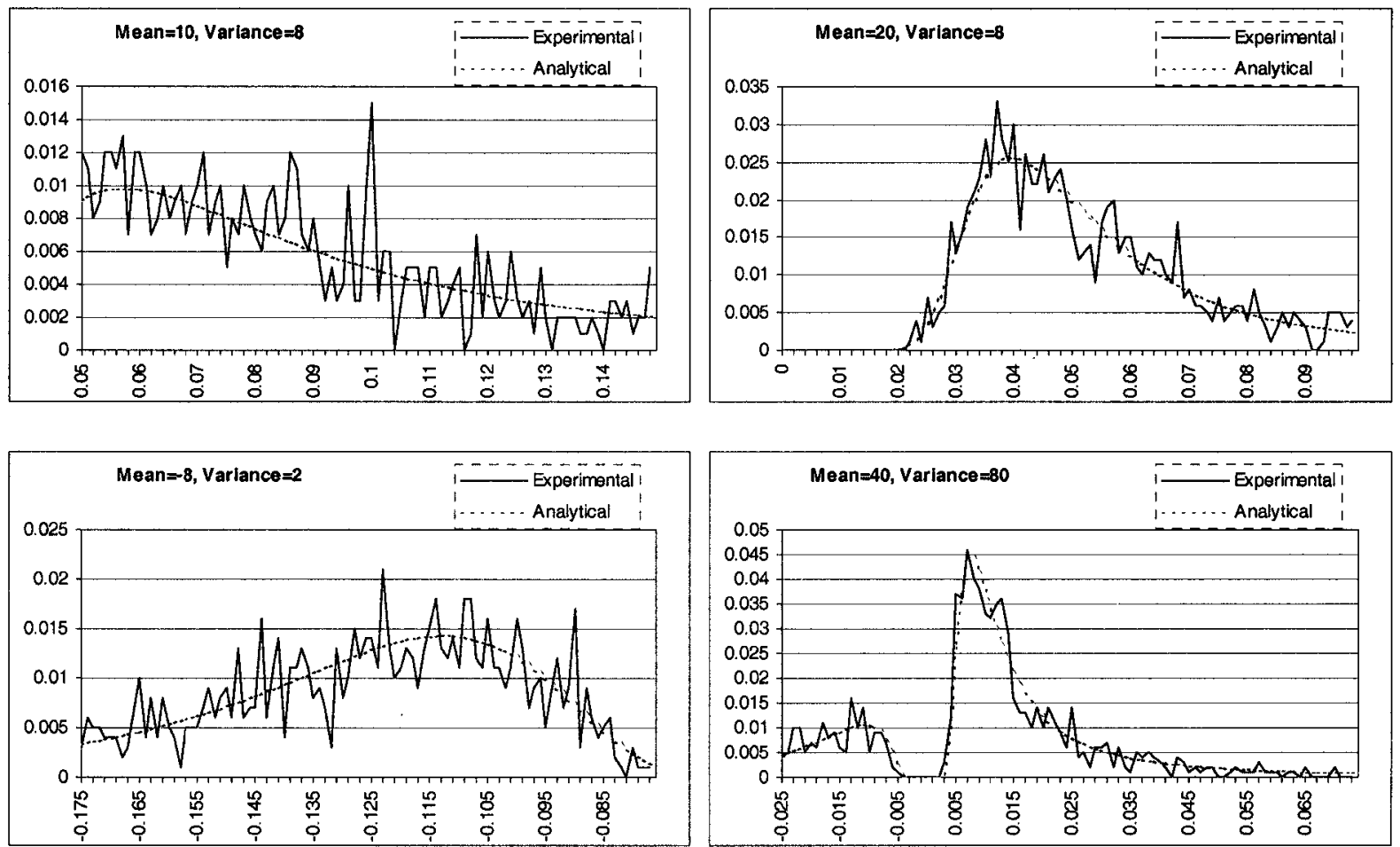

\section{Figure 11. Reciprocal Gaussian random variable}

As it can be seen in Figure 11, the reciprocal pdf changes its shape depending of the mean and variance of the originating Gaussian variable. If the mean is close to zero, and the variance has a small value, many of the Gauss random variable realizations are very close to zero, and the reciprocal will get very large values, positive or negative. This singularity makes difficult the experimental evaluation of the mean and variation of the reciprocal Gaussian random variable.

To determine the mean of the reciprocal Gaussian random variable through analytical methods, the following integral would need to be resolved:

$$
m_{Y}=\int y f_{Y}(y) d y=\int_{-\infty}^{\infty} \frac{1}{\sqrt{2 \pi} \sigma_{X} y} e^{-\frac{\left(1-m_{X} y\right)^{2}}{\sigma_{X}^{2} y^{2}}} d y
$$

However we have not found a method to solve this integral to a closed form. 


\section{COMPUTER SIMULATION}

The analytical results in Section 3 have been validated through computer simulations. In this section the mathematical model for the simulation is presented, as well as the simulation results obtained. Since, as discussed earlier, the effects of shadowing are difficult to investigate analytically, we have also used computer modeling to study this aspect.

\subsection{System Model for Computer Simulation}

We consider a system consisting of one BS and a collection of FRS which are connected wirelessly to the BS. Network elements - one BS and 121 FRS's - are placed on a hexagonal grid. The relays have a double role - to relay radio packets between the base station and other relays (thus forming a feeder system) and to provide access services to mobile terminals. The simulation is focused on the feeder system functionality. The BS is located in the center of the grid in location 60 , see Figure 12. The cell size of the hexagonal grid and implicitly the relative positions of the FRSs is adjusted function of the coverage radius of the FRS for MT access, such a way there is $100 \%$ coverage of the service area with minimum overlap. The FRS radius can be set as a simulation parameter. We define the attribute "in service" for each FRS, with value "true" if the FRS is located within a predetermined distance from the BS, called BS radius, and which can be set as a simulation parameter, and "false" otherwise. The number of "in service" FRS varies depending on the values of the BS and FRS radiuses, thus allowing the simulation program to be applied to a wide range of deployment scenarios. In Figure 12, the "in service" relays are depicted using a red triangle, while all relay locations are indicated by their number. 
To distribute the relays in the correct positions on the hexagonal grid, the horizontal and vertical coordinates for each relay, $x_{i}$ and $y_{i}$ respectively, $i=0,1,2, \ldots 120$, are calculated with the following expressions:

$$
\begin{aligned}
& x_{i}=\sqrt{3}\left(q u o t\left(\frac{i}{11}\right)-5\right) r \\
& y_{i}=\frac{3}{2}\left(\operatorname{rem}\left(\frac{i}{11}\right)-5\right) r
\end{aligned}
$$

where "quot" and "rem" represent the quotient and the remainder of the integer division, respectively, and $r$ is the coverage radius for each relay (also the grid hexagon radius). This arranges the 121 relays to be distributed on a hexagonal grid on an 11 by 11 matrix. We set the BS location at $i=60$, with coordinates $x_{60}=0, y_{60}=0$.

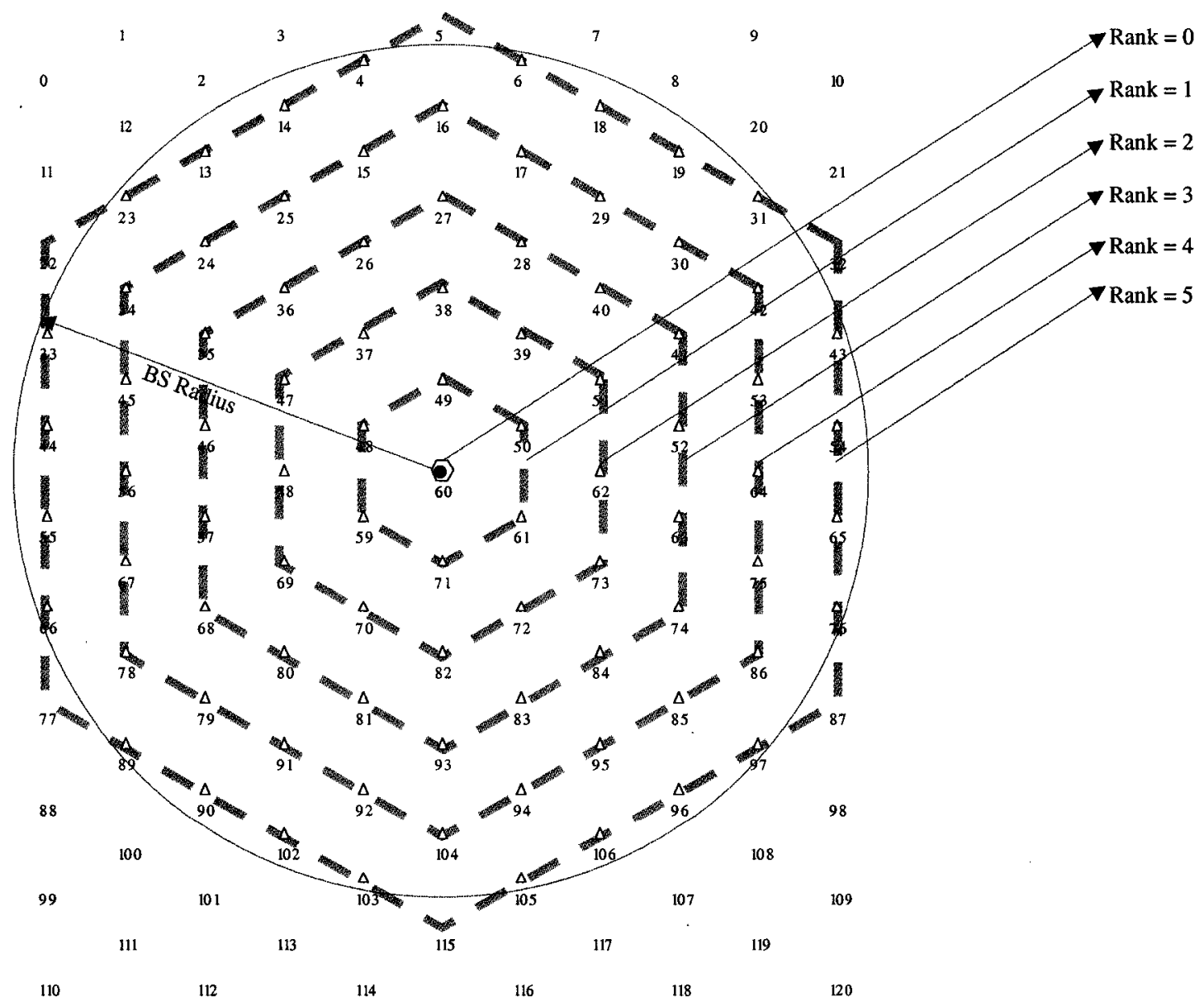

Figure 12. System layout on a hexagonal grid 
The relays are logically organized in concentric tiers around the BS using the hexagonal grid, see Figure 12. Each tier is indexed by its rank, starting with Rank $=0$ for the BS and ending with Rank $=7$ for the $7^{\text {th }}$ tier. We define a rank vector, which holds the rank of each relay:

$\begin{aligned} & \text { FRSrank }[121]=\quad\{7,7,6,6,5,5,5,6,6,7,7, \\ & 6,6,5,5,4,4,4,5,5,6,6, \\ & 5,5,4,4,3,3,3,4,4,5,5 \\ & 5,4,3,3,2,2,2,3,3,4,5 \\ & 5,4,3,2,1,1,1,2,3,4,5, \\ & 5,4,3,2,1,0,1,2,3,4,5, \\ & 5,4,3,2,2,1,2,2,3,4,5 \\ & 5,4,4,3,3,2,3,3,4,4,5 \\ & 6,5,5,4,4,3,4,4,5,5,6, \\ & 7,6,6,5,5,4,5,5,6,6,7, \\ &8,7,7,6,6,5,6,6,7,7,8\} .\end{aligned}$

The path loss model for each link includes log-distance model and normal shadowing [21]. The formula used to calculate the path loss for each link is:

$$
\operatorname{PathLoss}[d B]=32.4+20 \log _{10} d_{0}+10 \propto \alpha p \log \frac{d}{d_{0}}+20 \log _{10} F+X \sigma,
$$

where the involved terms are:

- $\quad 32.4$ is the free space loss in $\mathrm{dB}$ for $1 \mathrm{~m}$, at a carrier frequency of $1 \mathrm{GHz}$

- $d_{0}$ is the reference close in distance (up to which we assume free space loss), [m]

- $\quad p$ - path loss exponent

$\alpha$ - path loss exponent reduction factor - applies to shorter intermediate hops in multi-hop links

- $d$-hop length , [m]

- $F$-carrier frequency, [GHz]

- $X$-computer generated Gaussian random variable, $E[X]=0, V A R[X]=1$

- $\sigma$ - standard deviation to characterize the log-normal shadowing model, $[\mathrm{dB}]$

The normal distributed random variable $X$ is independently generated for each path using the following expression [23]:

$$
X=\sqrt{-2 \ln x_{1}} \cos \left(2 \pi x_{2}\right)
$$


where $x_{1}$ and $x_{2}$ are two random numbers uniformly distributed between 0 and 1 . For each link, the path loss is stored in a path-loss vector and reused as needed until the end of the simulation. The size of the path-loss vector is set to the maximum number of path loss values which would need to be stored, which is $121 * 120 / 2=7260$. This results in uncorrelated shadowing from relay to relay (even for relays along same geometrical direction).

The signal to noise ratio for each receiver is calculated using the formula

$$
S N R[d B]=10 \log _{10} P_{T}+G_{T}+G_{R}-\text { PathLoss }-10 \log _{10}\left(1.38 \cdot 10^{-23} \cdot 300 \cdot B\right)-N F
$$

- $\quad P_{T}-$ transmit power, $[\mathrm{W}]$

- $G_{T}-$ transmit antenna gain, $[\mathrm{dB}]$

- $G_{R}$ - receive antenna gain, $[\mathrm{dB}]$

- $1.38 \times 10^{-23}-$ Boltzman constant, $[\mathrm{J} / \mathrm{K}]$

- 300 - absolute temperature, $[\mathrm{K}]$

- $\quad B$ - channel bandwidth, $[\mathrm{Hz}]$

- $\quad N F-$ receiver noise figure, $[\mathrm{dB}]$

Using the look-up table shown in Table 2, each link is allocated a modulation scheme with highest spectral efficiency allowed by its SNR. The upper limit of the spectral efficiency range is capped at $8 \mathrm{bps} / \mathrm{Hz}$.

\begin{tabular}{|c|c|}
\hline 0 & 0.1 \\
\hline 4 & 1 \\
\hline 6 & 1.33 \\
\hline 6.8 & 1.5 \\
\hline 7.8 & 1.75 \\
\hline 10 & 2 \\
\hline 12 & 2.67 \\
\hline 13 & 3 \\
\hline 15 & 3.5 \\
\hline 17.7 & 4 \\
\hline 19 & 4.5 \\
\hline 21 & 5.25 \\
\hline 26 & 6 \\
\hline 31 & 7 \\
\hline 35.5 & 8 \\
\hline
\end{tabular}

Table 2. Spectral efficiency vs. SNR 
A separate individual message of $1 \mathrm{Mbit}$ (arbitrary value) is sent to all relays located within a specified radius (BS service range) around the BS, simulating traffic in a real network with mobile terminals serviced by each relay.

The individual time required to transmit the message along a given hop from the transmitting to the receiving relay, is calculated as

$$
M T T_{h o p}=\frac{10^{6}}{B \eta}
$$

where

- $M T T_{\text {hop }}$ - message transfer time, [s]

- $\quad B$ - channel frequency bandwidth, $[\mathrm{Hz}]$

- $\quad \eta$-spectral efficiency for the respective hop, [bit/s/Hz].

The total time required for all relays to receive their messages is calculated as

$$
M T T_{\text {Total }}=\sum_{i=1}^{121} I_{i} \sum_{j}^{n_{i}} M T T_{\text {hop }_{i j}},
$$

where:

- $I_{i}$ is an "in service" indicator, $I_{i}=1$ if the relay $i$ is within the BS radius, and $I_{i}=0$ otherwise.

- $n_{i}$ is the number of hops required to transmit the message to relay $i$

The ultimate objective of the network deployment strategy optimization is to save resources by improving the aggregate spectral efficiency (per link) and average spectral efficiency (per entire network). The formula for calculating the average spectral efficiency is

$$
\eta=\frac{10^{6} n_{s}}{M T T_{\text {total }} B}
$$

- $\quad \eta$ - average network spectral efficiency, $[\mathrm{bit} / \mathrm{s} / \mathrm{Hz}]$

- $n_{s}$ - number of relays in service (equivalent with number of relays per BS)

- $\quad B$ - channel bandwidth, $[\mathrm{Hz}]$ 
We assume that any relay can communicate with any relay and with the BS. When the BS has a message to be transmitted to a specific relay, the message can be passed from relay to relay until its destination. We want to determine the most efficient method of transmission, such that the aggregate message transfer time is minimized. We consider 4 methods in which a message can be routed to its destination relay:

- Single-hop transmission

- Greedy multi-hop transmission

- Using multi-hop criterion

- Exhaustive search

The computer simulation consisted in running all 4 routing methods listed above, and comparing the results obtained.

\subsubsection{Method 1 - Single-hop transmission}

The message is sent to its destination relay directly in a single hop. For each link, the computer generates the log-normal shadowing component, calculates the path loss, the SNR and maximum spectral efficiency, then the message transfer time. An example of network topology in this case is shown in Figure 13.

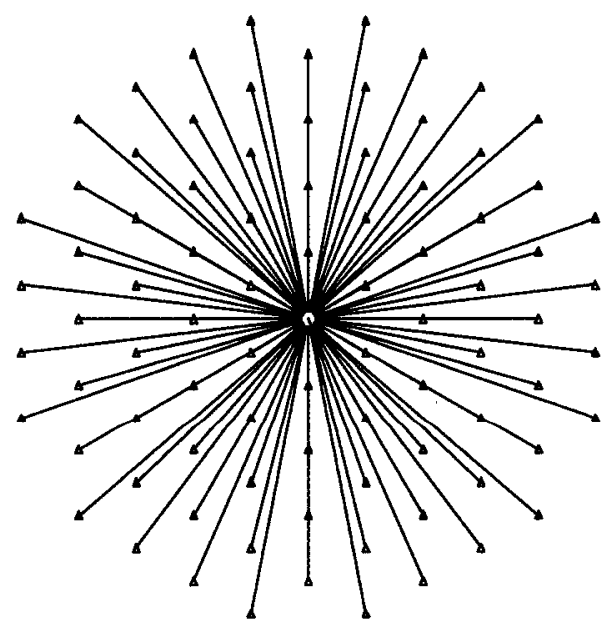

Figure 13. Example of network topology, direct single-hop transmission 
Each single path SNR is memorized for future use in case the same hops are part of the routes analyzed in subsequent methods.

\subsubsection{Method 2 - Greedy multi-hop transmission}

This method would be the opposite of the single-hop - use multi-hop transmission, increasing the number of hops to the maximum extent possible. In order to obtain efficient and short routes, the following algorithm is used for the route selection process:

Since the communication from the BS to the relays takes place in a "radial" direction from BS, the difference between the ranks of relays communication over any hop is always 1, i.e., the two relays are located on adjacent concentric tiers, see Figure 12.

We want to avoid hops with heavy shadowing - otherwise there would not be much practical purpose of using multi-hop transmission. As such, the next relay along the multi-hop route is selected such a way to minimize MTT. We define the cost for each hop as the MTT for the respective hop. We start from the $\mathrm{BS}$, and obviously the relays in $1^{\text {st }}$ tier are connected directly to the BS. Next, for each route to connect one of the relays of rank 2 ( $2^{\text {nd }}$ tier), we choose as intermediate station any of the rank 1 relays which results in the smallest sum of transfer times over the two hops. Once a route between BS and a relay is selected, it will be used by all traffic for that relay or traffic using that relay as an intermediate. Thus, we assign the aggregate $M T T=$ $M T T_{1}+M T T_{2}$ to the relay, which will be used to evaluate costs for other possible routes to relays of ranks 3, 4, etc. The same process is repeated for all in service relays, generating a tree structure in a general radial form. This algorithm is somehow similar to the Bellman-Ford algorithm for finding shortest path routes [24]. The complete logical flowchart for the algorithm is shown in Figure 14. 


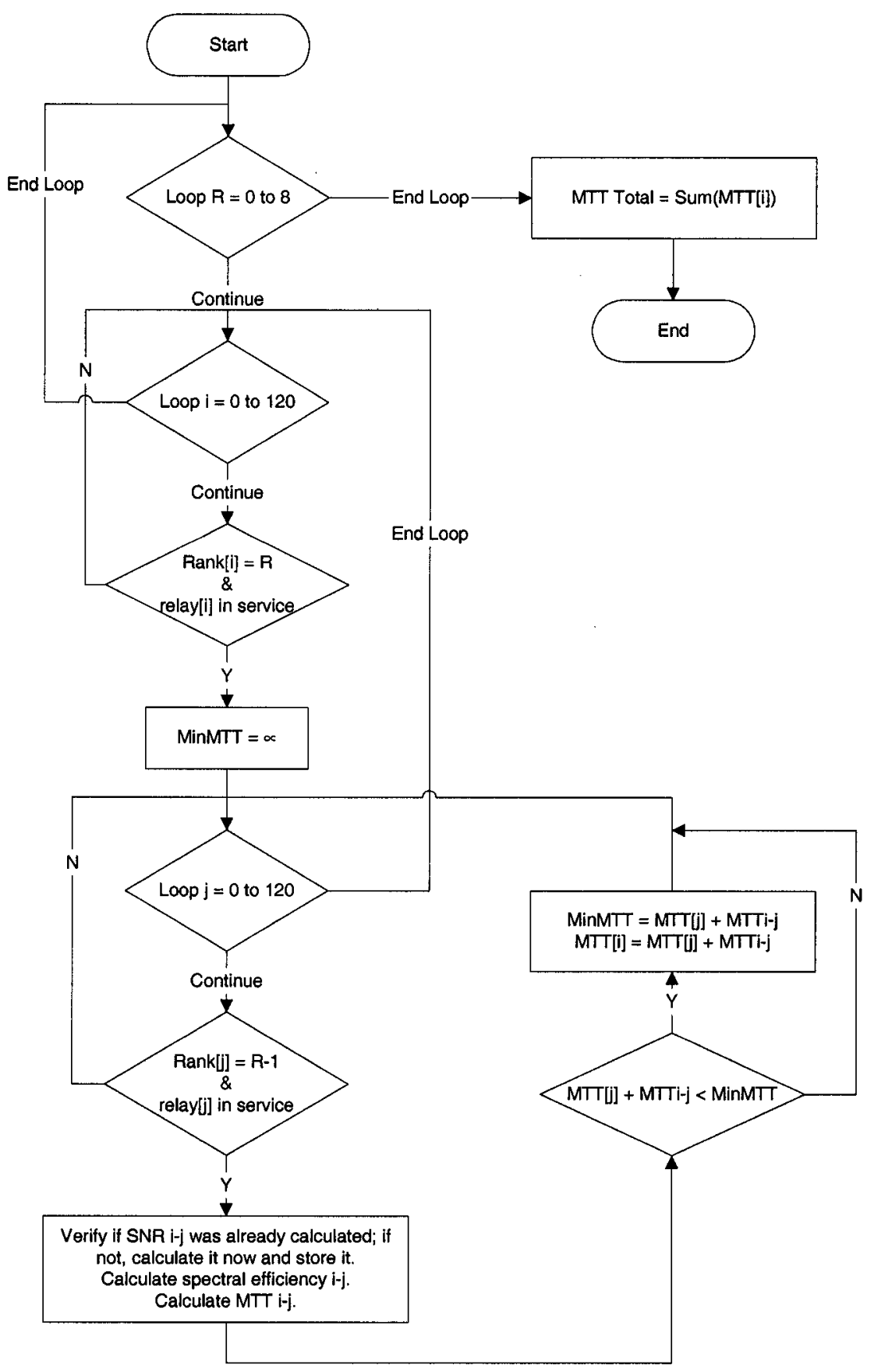

\section{Figure 14. Greedy multi-hop transmission - algorithm flowchart}

The resulting network transmission topology is a radial tree without loops, see the example in Figure 15. In the diagram, which considers only downlink transmission, transmitters are symbolized by hexagons, while receivers are symbolized by triangles. As it can be seen, the transmission tree limbs are in general short, between relays located in adjacent positions. The 
multi-hop links are organized in hops which are always between relays located on adjacent tiers. As the algorithm "goes for" short links with high SNR, and high spectral efficiency, it can be considered greedy (or opportunistic).

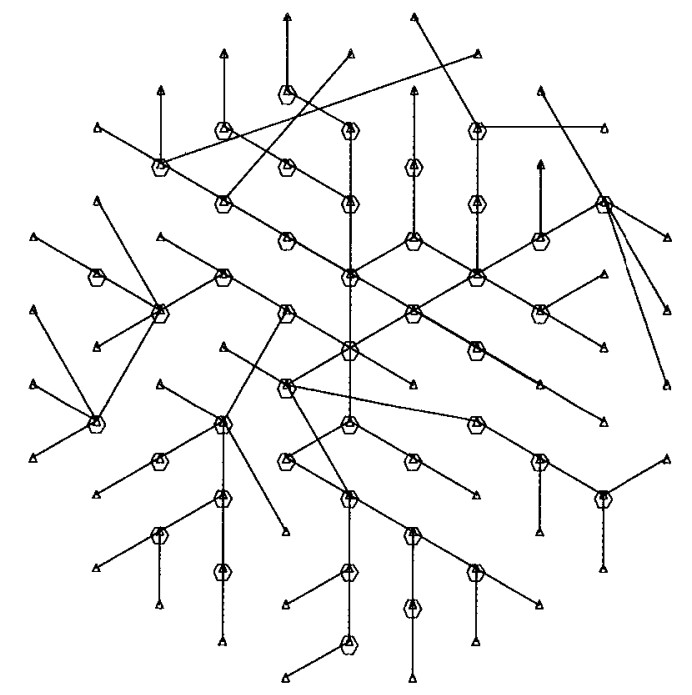

Figure 15. Example of network topology, greedy multi-hop transmission

\subsubsection{Method 3 - Multi-Hop transmission using Multi-Hop Criterion (MHC)}

Earlier in Section 3.5, introducing the MHC, we have shown that multi-hop links are not in all cases optimal from an aggregate spectral efficiency point of view. Depending on the specific conditions for each link, a single-hop link may or may not be replaced by a more efficient multihop link. Also, for the multi-hop links, sometimes a reduced number of hops may achieve a higher aggregate spectral efficiency.

Generally, Method 3 is similar to Method 2, only here we want to reduce the number of hops as dictated by the MHC. Instead having a hop for each time a packet is crossing from one rank to the next (or from a hexagonal tier to the next), here the hops may be longer and pass over one or more ranks. 
Once the SNR for the single hop link between the BS and the destination relay is known, using MHC equation (34) we can find the number of hops at which a more efficient multi-hop link becomes feasible. Knowing the target number of hops for the multi-hop link, and the rank of the destination relay, we calculate the size of each hop, in "rank units". In other words, we determine how many hexagonal tiers should be crossed by each hop. We use the following formula:

$$
\text { HopSize }\left[\text { ranks] }=\operatorname{int}\left(\frac{\operatorname{Rank}_{\text {dest }}}{n_{M H C}}\right)\right.
$$

- $\operatorname{Rank}_{\text {dest }}$ - the rank of the destination relay

- $\quad n_{M H C}-$ number of hops according to MHC criterion

- The int function returns the closest integer to its argument

Once the hop size is known, we use the same algorithm as in the Method 2, with the following difference: assuming the destination relay has rank $R_{n}$, instead of choosing the "best" intermediate relay from the relays located in the tier of next lower rank $R_{n}-1$, we select the "best" intermediate relay from the tier of rank $R_{n}-$ HopSize. The detailed procedure to determine the number of hops using MHC is shown in Figure 16.

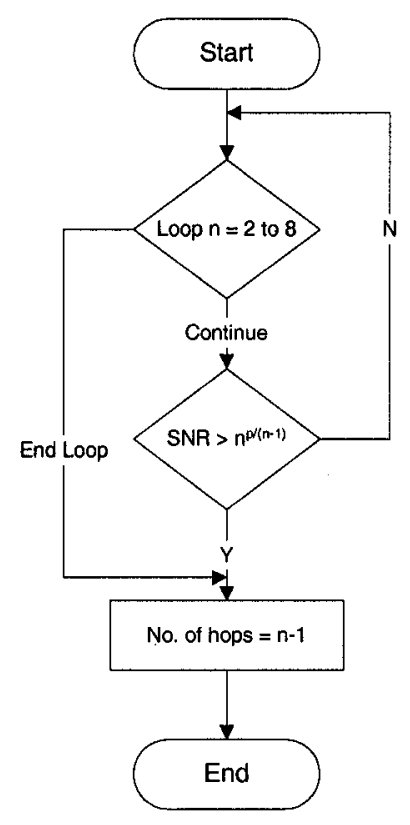

Figure 16. Algorithm to determine number of hops using MHC 
The complete logical flowchart for the algorithm is shown in Figure 17.

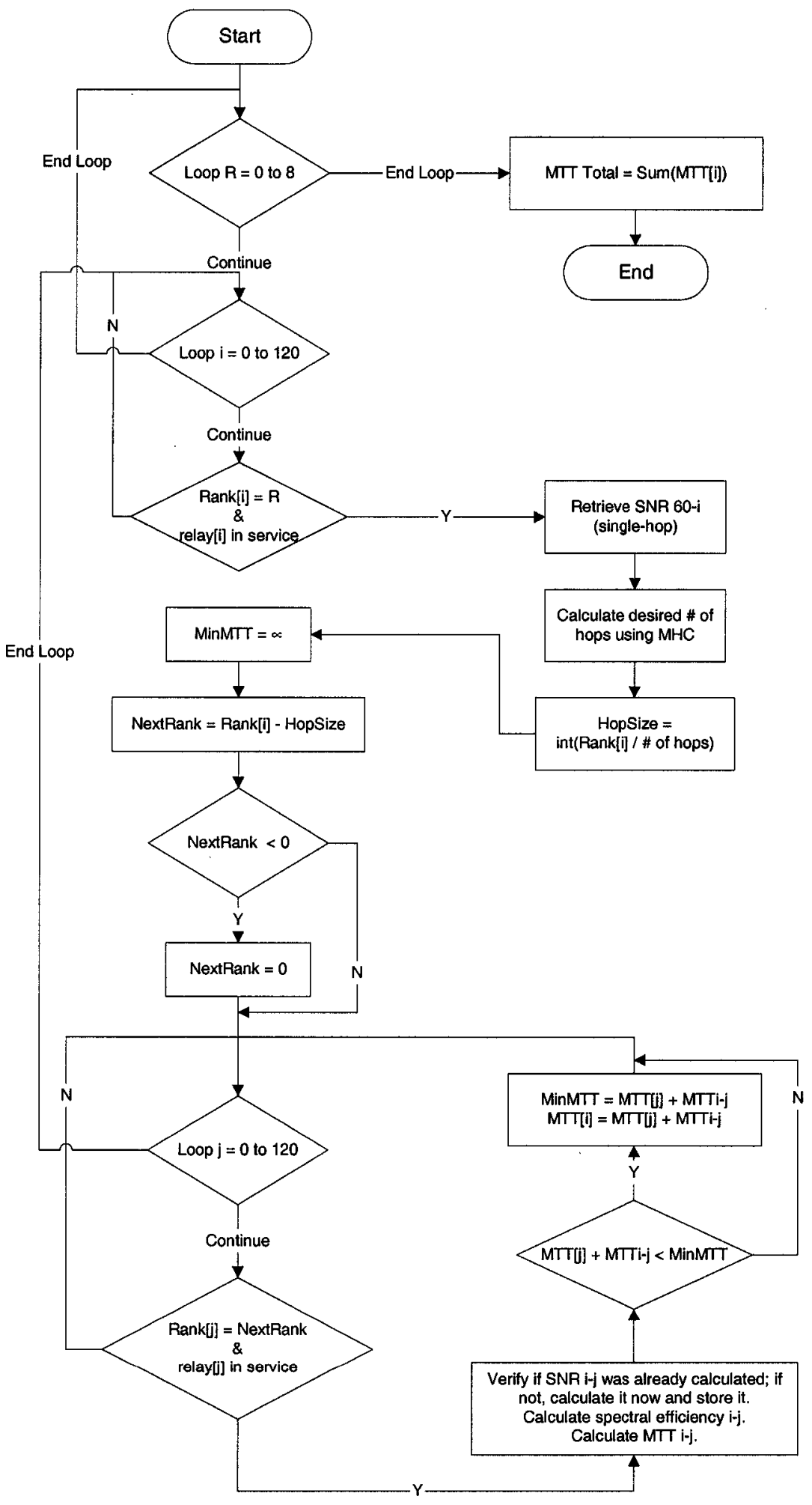

Figure 17. Multi-hop transmission using MHC - algorithm logical flowchart 
The resulting network topology, an example shown in Figure 18, is a loop-less tree. With similar propagation parameters, the tree has longer limbs; some relays of rank larger than one are connected directly to the BS - in these cases, the MHC indicated the single hop link achieves a better aggregate spectral efficiency.

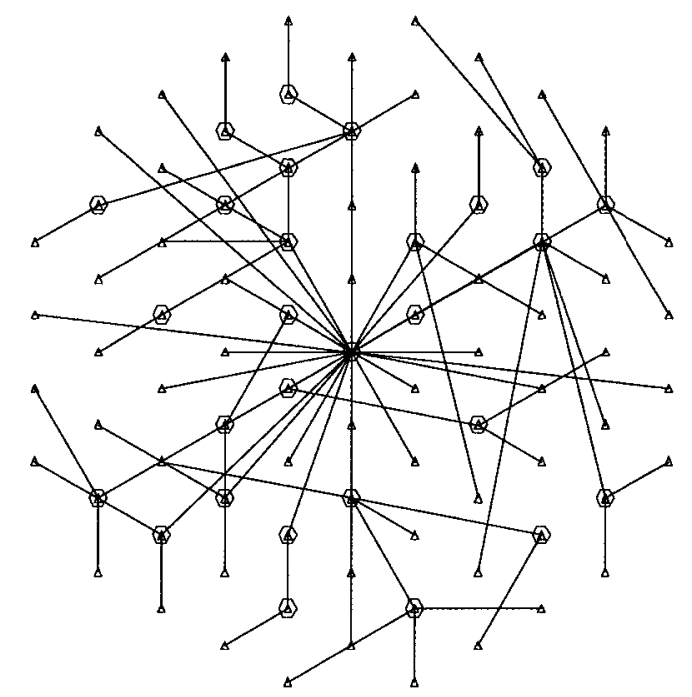

Figure 18. Network topology - transmission using MHC

\subsubsection{Method 4 - Exhaustive search}

In the same fashion as in the previous methods, this search method assigns costs to each relay, starting from the BS and working toward the network periphery. A link cost is represented by the MTT across a hop between two relays. The BS is assigned zero cost. For each relay $i$, we calculate the sum between the cost of any relay $k$ of equal or lower rank (already available) and the cost of the link $i-k$. We repeat, calculating the sum for all intermediate relays $k$. The smallest sum obtained is assigned as the cost for $i$, and the chosen relay $k$ is part of the route from the relay $i$ toward the $\mathrm{BS}$.

The algorithm ensures that the selected routes are optimal and the cost assigned to each relay (actually the total MTT from the BS to the relay) is the smallest possible. 


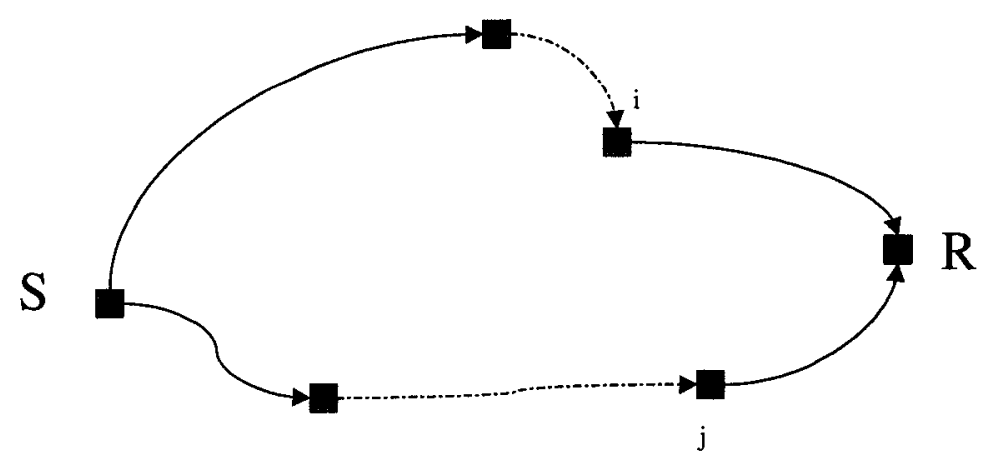

Figure 19. Simplified network topology

\section{Proof:}

Consider the diagram in Figure 19. Assume that using the method described above, we select the route $\mathrm{S}-\ldots-\mathrm{i}-\mathrm{R}$ as the minimum cost route for the destination relay $\mathrm{R}$. Now, let us suppose that this is incorrect, and the best route with the lowest cost is another route, for example S-...-j$R$. This would mean that sum of the costs of the route portions $S-\ldots-j$ and $j-R$ must be lower than costs of S-...-i and $\mathrm{i}-\mathrm{R}$, which contradicts the starting assumption.

The complete logical flow chart for the algorithm is shown in Figure 20 


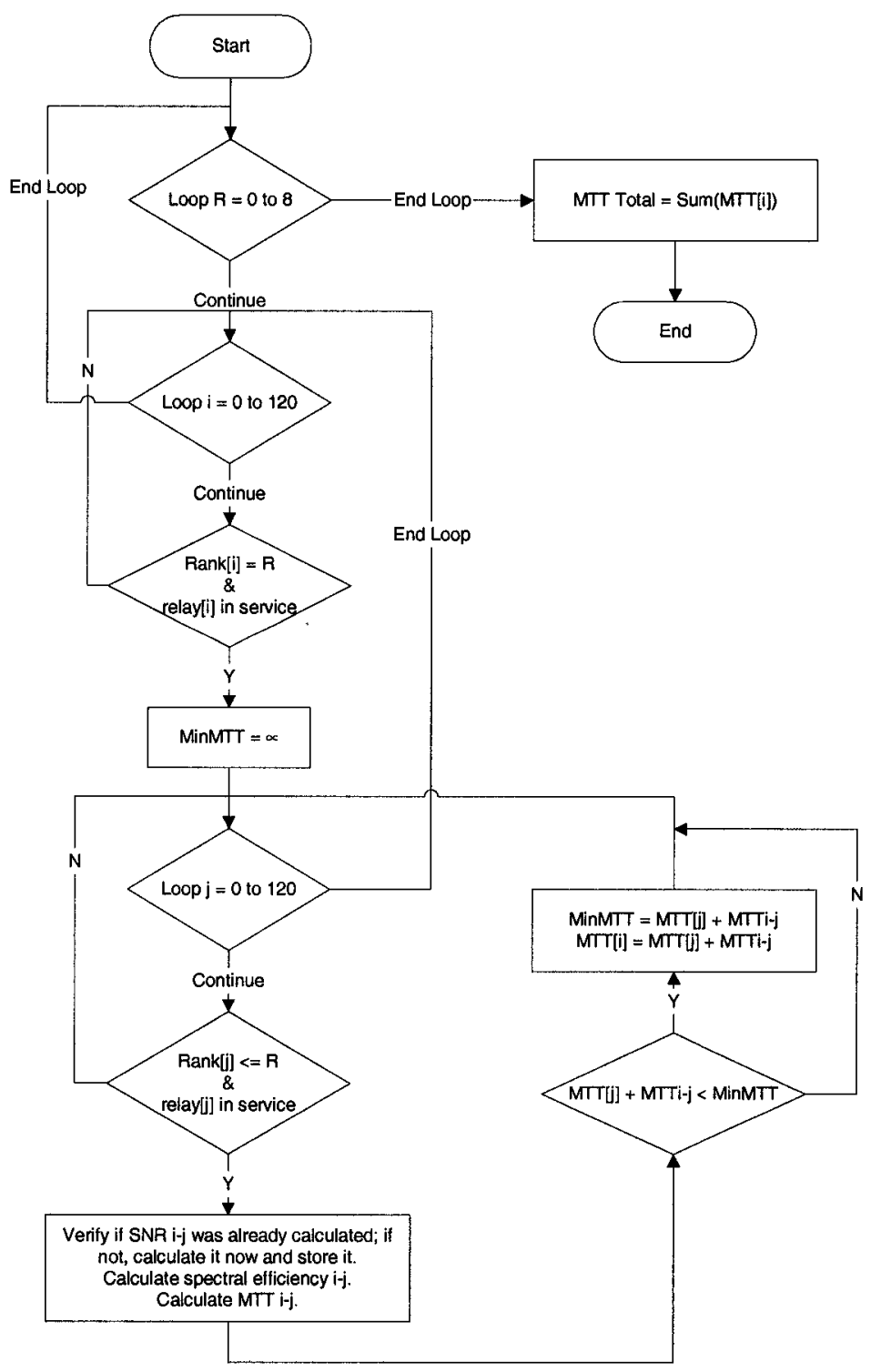

Figure 20. Algorithm flowchart - exhaustive route search

A example of network configuration resulted from using method 4 is shown in Figure 21. In appearance, the topology is the most "disorganized" from the 4 methods. This is due to the fact that the algorithm has the freedom to choose from a wider pool of intermediate relays. Direct comparisons can be made between Figure 15, Figure 18, Figure 21, as the same shadowing values are used for all. 


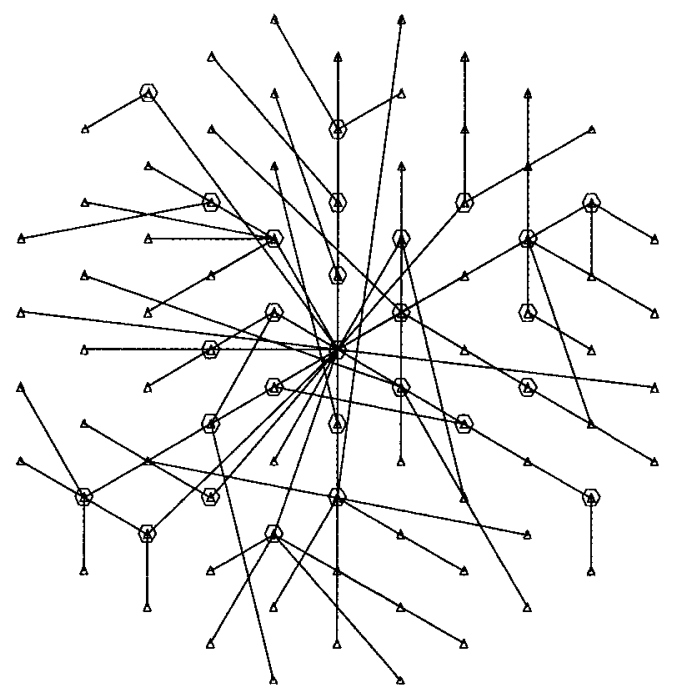

\section{Figure 21. Network topology, exhaustive search}

As mentioned before, methods 2 to 4 above are deployed from the BS going outward toward the periphery. This could also be thought of as a trellis search for the path with the smallest cost, where the cost is the message transfer time.

\subsection{Simulation Results}

The mathematical model described in the previous section was implemented in $\mathrm{C}$ code. The complete set of simulation parameters and their range of values is shown in Table 3:

\begin{tabular}{|c|l|c|l||}
\hline PARAMETER & \multicolumn{1}{|c|}{ DESCRIPTION } & UNITS & \multicolumn{1}{|c||}{ RANGE OF VALUES } \\
\hline $\mathrm{R}$ & BS service radius & $\mathrm{m}$ & 800 \\
\hline $\mathrm{r}$ & Relay service radius & $\mathrm{m}$ & 100,150 \\
\hline $\mathrm{F}$ & Carrier frequency & $\mathrm{GHz}$ & 5 \\
\hline $\mathrm{B}$ & Channel bandwidth & $\mathrm{MHz}$ & 25 \\
\hline $\mathrm{P}_{\mathrm{t}}$ & Transmit power & $\mathrm{W}$ & 1 \\
\hline $\mathrm{G}_{\mathrm{t}}$ & Transmit antenna gain & $\mathrm{dB}$ & 10 \\
\hline $\mathrm{G}_{\mathrm{r}}$ & Receive antenna gain & $\mathrm{dB}$ & 10 \\
\hline $\mathrm{d}_{0}$ & Reference close-in distance & $\mathrm{m}$ & 2 \\
\hline
\end{tabular}




\begin{tabular}{||c|l|l|l||}
\hline \hline $\mathrm{p}$ & Path loss exponent & & $3,3.25,3.5,3,75,4$ \\
\hline$\sigma$ & $\begin{array}{l}\text { Standard deviation log- } \\
\text { normal shadowing }\end{array}$ & $\mathrm{dB}$ & $0,5,8,10$ \\
\hline$\alpha$ & $\begin{array}{l}\text { Reduction coefficient path } \\
\text { loss exponent }\end{array}$ & $1,0.8$ \\
\hline $\mathrm{NF}$ & Receiver noise figure & $\mathrm{dB}$ & 5 \\
\hline
\end{tabular}

Table 3 - Simulation Parameters

\subsubsection{Same path loss exponent for all links}

In this section we present sets of plots of average network spectral efficiency versus path loss exponent, for different values of the standard deviation for log-normal shadowing. In order to avoid "skewed" results due to randomness, and, reduce the confidence interval for the results, all scenarios are repeated 100 times and the results averaged.

The relay radius is assumed $100 \mathrm{~m}$ in Figure 22, and $150 \mathrm{~m}$ in Figure 23. A larger relay radius results in a wider spacing between relays, amounting to a smaller number of relays located within the coverage area of a BS and serviced by it. The following observations can be made:

1. As expected the spectral efficiency decreases for higher path loss exponents.

2. Although on a per link basis the spectral efficiency can go as high as $8 \mathrm{bit} / \mathrm{s} / \mathrm{Hz}$, the average network spectral efficiency is much lower, (close to $1 \mathrm{bit} / \mathrm{s} / \mathrm{Hz}$ for high values of path loss exponent $p$ ), regardless of the routing method used. This is due to repeated resource assignment, using each time a different channel, to carry the same information (radio packet) over a number of hops to the relays located close to network periphery, according with our initial channel orthogonality assumption. This is consistent with the result presented in Section 3.2. 

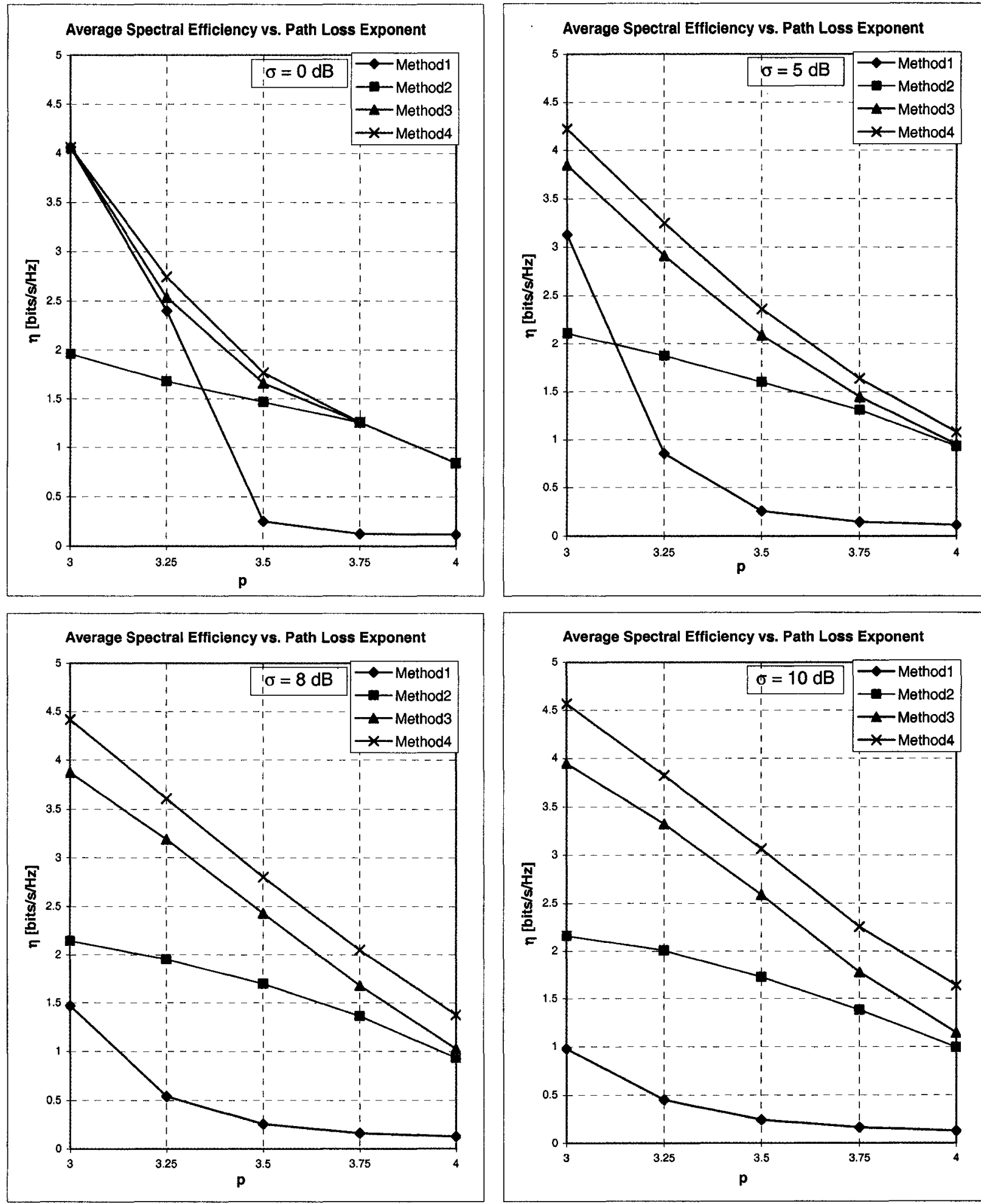

\begin{tabular}{|c|c|c|c|c|c|c|c|c|c|c|c|}
\hline$R$ & $\mathrm{r}$ & $\mathrm{F}$ & $\mathrm{B}$ & $\mathrm{Pt}$ & $\mathrm{NF}$ & $\mathrm{Gt}$ & $\mathrm{Gr}$ & $\mathrm{d} 0$ & $\mathrm{p}$ & $\sigma$ & $\alpha$ \\
\hline $800 \mathrm{~m}$ & $100 \mathrm{~m}$ & $5 \mathrm{GHz}$ & $25 \mathrm{MHz}$ & $1 \mathrm{~W}$ & $5 \mathrm{~dB}$ & $10 \mathrm{~dB}$ & $10 \mathrm{~dB}$ & $2 \mathrm{~m}$ & & & 1 \\
\hline
\end{tabular}

Figure 22. Average network spectral efficiency, relay service radius $100 \mathrm{~m}$ 

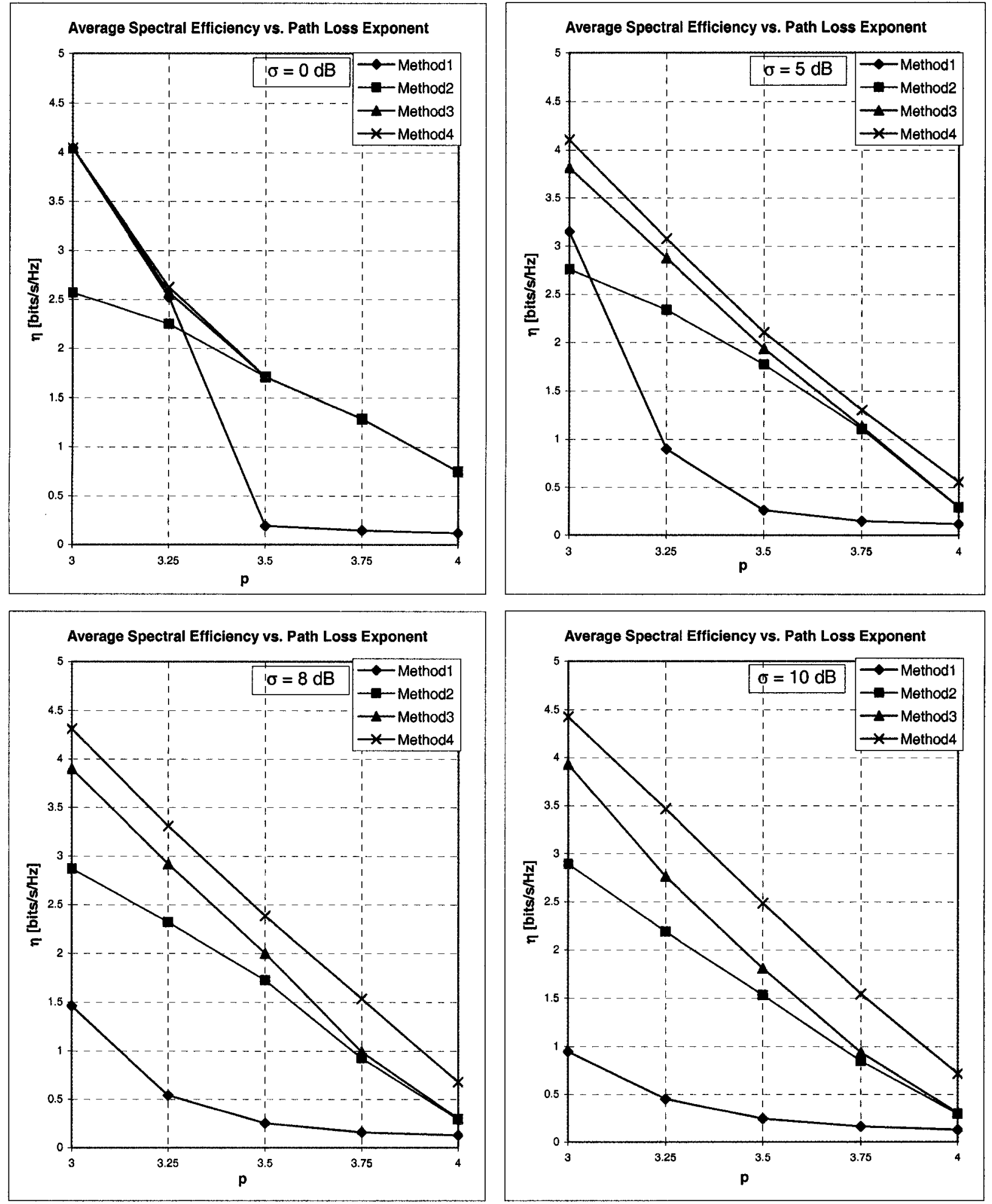

\begin{tabular}{|c|c|c|c|c|c|c|c|c|c|c|c|}
\hline$R$ & $r$ & $F$ & $B$ & $P t$ & $N F$ & $G t$ & $G r$ & do & $p$ & $\sigma$ & $\alpha$ \\
\hline $800 \mathrm{~m}$ & $150 \mathrm{~m}$ & $5 \mathrm{GHz}$ & $25 \mathrm{MHz}$ & $1 \mathrm{~W}$ & $5 \mathrm{~dB}$ & $10 \mathrm{~dB}$ & $10 \mathrm{~dB}$ & $2 \mathrm{~m}$ & & & 1 \\
\hline
\end{tabular}

Figure 23. Average network spectral efficiency, relay service radius $150 \mathrm{~m}$ 
3. On the other side, we can observe that the scheme with a reduced number of relays ( $r$ $=150 \mathrm{~m}$ ) achieves a lower average spectral efficiency, especially at high path loss values. This is because the longer hop lengths prohibit high modulation rates, leading to lower spectral efficiency. We conclude the distance between relays (grid size) and the rest of the system parameters (transmit power, antenna gains, etc) must be chosen such a way to enable maximum data rates at least between adjacent grid locations.

4. For high values of the path loss exponent $p$, the Method 1 using single-hop links has the lowest performance. Its average spectral efficiency performance tends asymptotically toward zero as the path loss exponent increases. Since the capacity decreases toward zero, we can conclude the communication is not possible using Method 1 at high path loss exponents. However, at low values of the path loss exponent, and especially when there is no shadowing, the direct single-hop link performance is optimal. This leads to the possibility of deploying feeder systems with fixed relays installed within LOS of base station, where particular terrain conditions would allow such deployment; with low path loss exponent and no shadowing, feeder systems of this kind would function very well using single-hop direct links, i.e., no multi-hop.

5. At low propagation exponent values (i.e., at high SNR) the Method 2 (greedy multihop) has the lowest spectral efficiency, due to unnecessary hoping. The highest possible modulation scheme considered allows for a hop spectral efficiency of 8 bit/sec/Hz, even when the hop SNR is higher than $35.5 \mathrm{~dB}$ (Table 2). Thus, using many short hops at SNR higher than the level required for the maximum modulation level is an inefficient use of resources.

6. The shadowing has a minimal impact on the performance of Methods 2, 3 and 4, while the direct single-hop transmission method is heavily affected. As described in the previous section, the algorithms for methods 2,3 and 4 avoid hops with heavy shadowing. This is the core advantage of multi-hop technology. 
7. At high path loss values and low shadowing, the algorithms 2,3 and 4 converge to the same solution, the spectral efficiency performance and the resulting routes (see Appendix ) being almost identical. This can be explained as follows: for low SNR values, the MHC (Method 2) will recommend using multi-hop links with the maximum number of hops (Method 3), which in this case is the optimal solution (Method 4). For higher shadowing values, the random variation in path losses between various relay locations advantages the Method 4 which has more flexibility in choosing the routes.

8. The Method 3, controlled by the MHC, has a performance closer to optimal (Method 4) at larger number of hops (i.e. smaller relay radius, $100 \mathrm{~m}$ ). This is due to the fact that the error generated by the approximation to an integer value (see Method 3 algorithm flowchart in Figure 17) is relatively more significant when the number of hops is small.

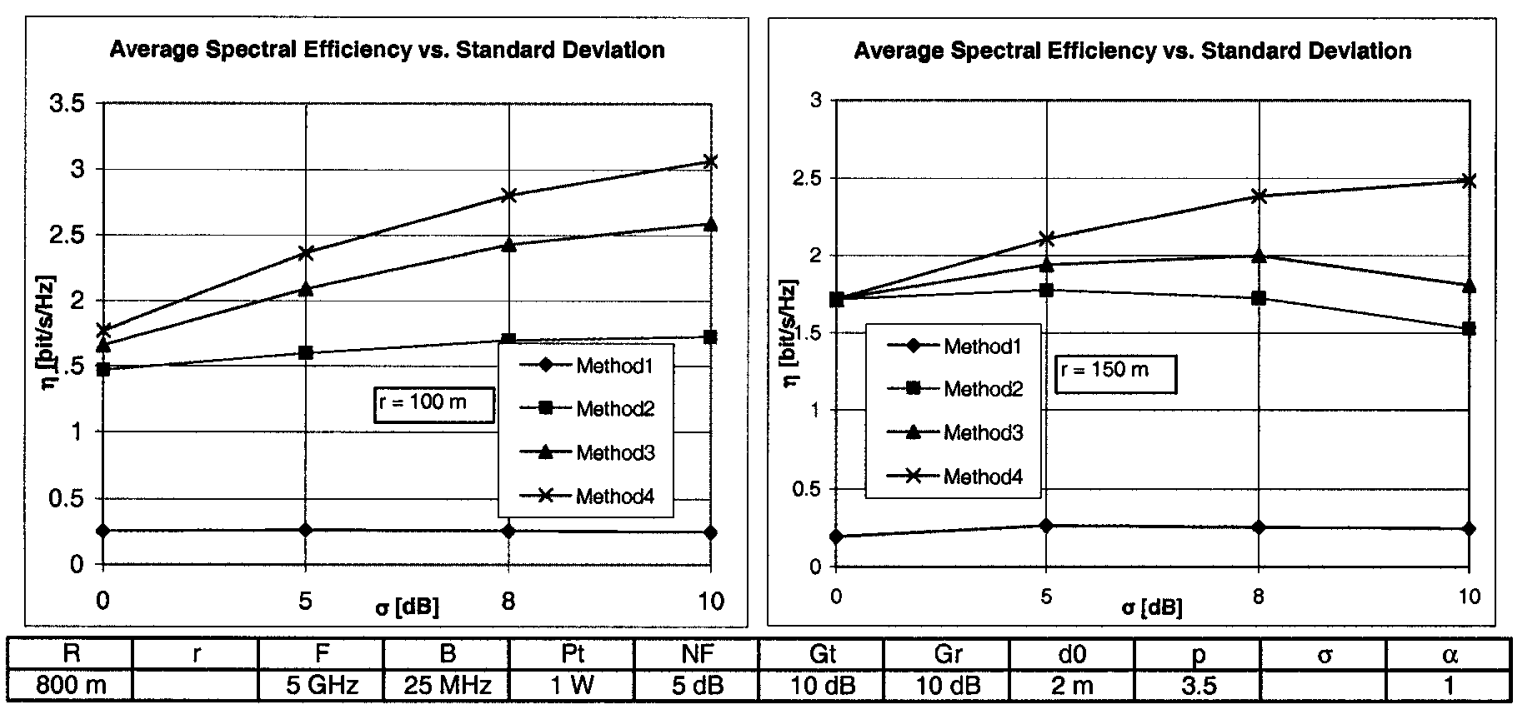

Figure 24. Average Spectral Efficiency vs. Standard deviation

9. An increase in average network spectral efficiency can be noticed for larger values of the standard deviation of the log-normal shadowing, see Figure 24. This is somehow counterintuitive, however it can be explained considering that the mathematical model for log-normal shadowing allows for variations from the median in both directions, 
i.e., for larger or smaller path losses. Whenever a below median path loss is realized in some link, Methods 2, 3 and 4 have the ability to exploit it and use it to minimize the path costs to that relay, and the path costs of any relay located downhill on the same branch of the transmission tree.

Samples of the network topology realizations for all routing methods are presented in Appendix. It can be observed that if $\sigma=0$, for low path loss exponent the MHC routing method results in an identical topology with the direct single-hop, while for high path loss MHC gives the same solution as the greedy (and the exhaustive search) methods. Also, it is apparent that some of the computer generated routing solutions, while targeted at achieving high average spectral efficiency, may not be ideal from a traffic engineering point of view (i.e., traffic is routed along the best route from spectral efficiency point of view, without regard to possible congestion).

\subsubsection{Reduced path-loss exponent value for short links}

In this section we attempt to simulate the scenario presented in section 3.5.1. A reduction factor $\alpha$ of the path loss exponent is applied only to the short hops. "Short hop" is arbitrarily defined in this context as a hop having the length no larger than $350 \mathrm{~m}$. Also, the algorithm in Figure 16 is modified to use the general formula of the multi-hop criterion, equation (39).

The variation of average spectral efficiency versus path loss exponent, when shorter hops have a reduction of the propagation exponent of $\alpha=0.8$, is presented in plots in Figure 25 and Figure 26. 

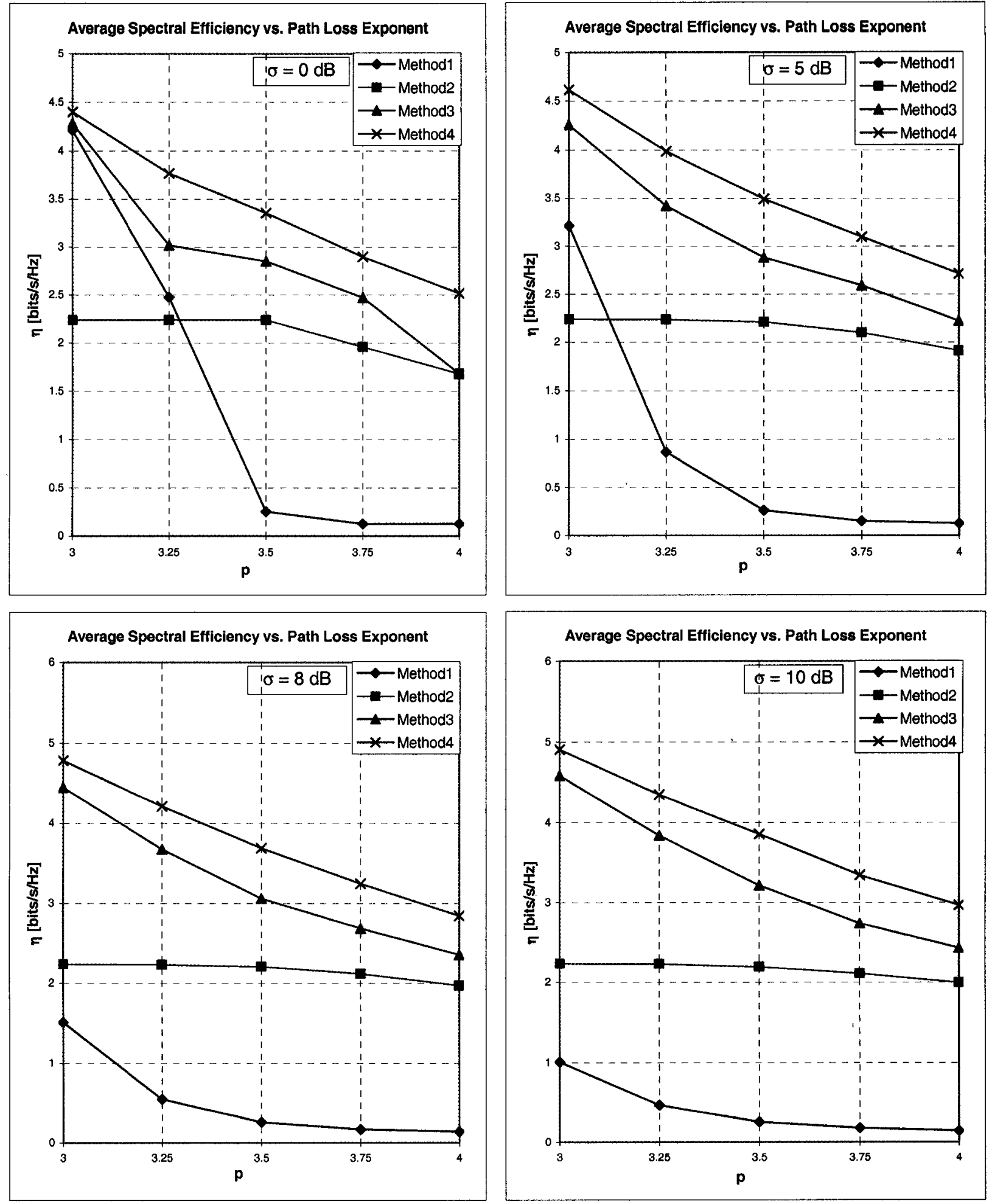

\begin{tabular}{|c|c|c|c|c|c|c|c|c|c|c|c|}
\hline$R$ & $r$ & $F$ & $B$ & $P t$ & $N F$ & $G t$ & $G r$ & $d 0$ & $p$ & $\sigma$ & $\alpha$ \\
\hline $800 \mathrm{~m}$ & $100 \mathrm{~m}$ & $5 \mathrm{GHz}$ & $25 \mathrm{MHz}$ & $1 \mathrm{~W}$ & $5 \mathrm{~dB}$ & $10 \mathrm{~dB}$ & $10 \mathrm{~dB}$ & $2 \mathrm{~m}$ & & & 0.8 \\
\hline
\end{tabular}

Figure 25. Average network spectral efficiency, relay service radius $100 \mathrm{~m}, \alpha=0.8$ 

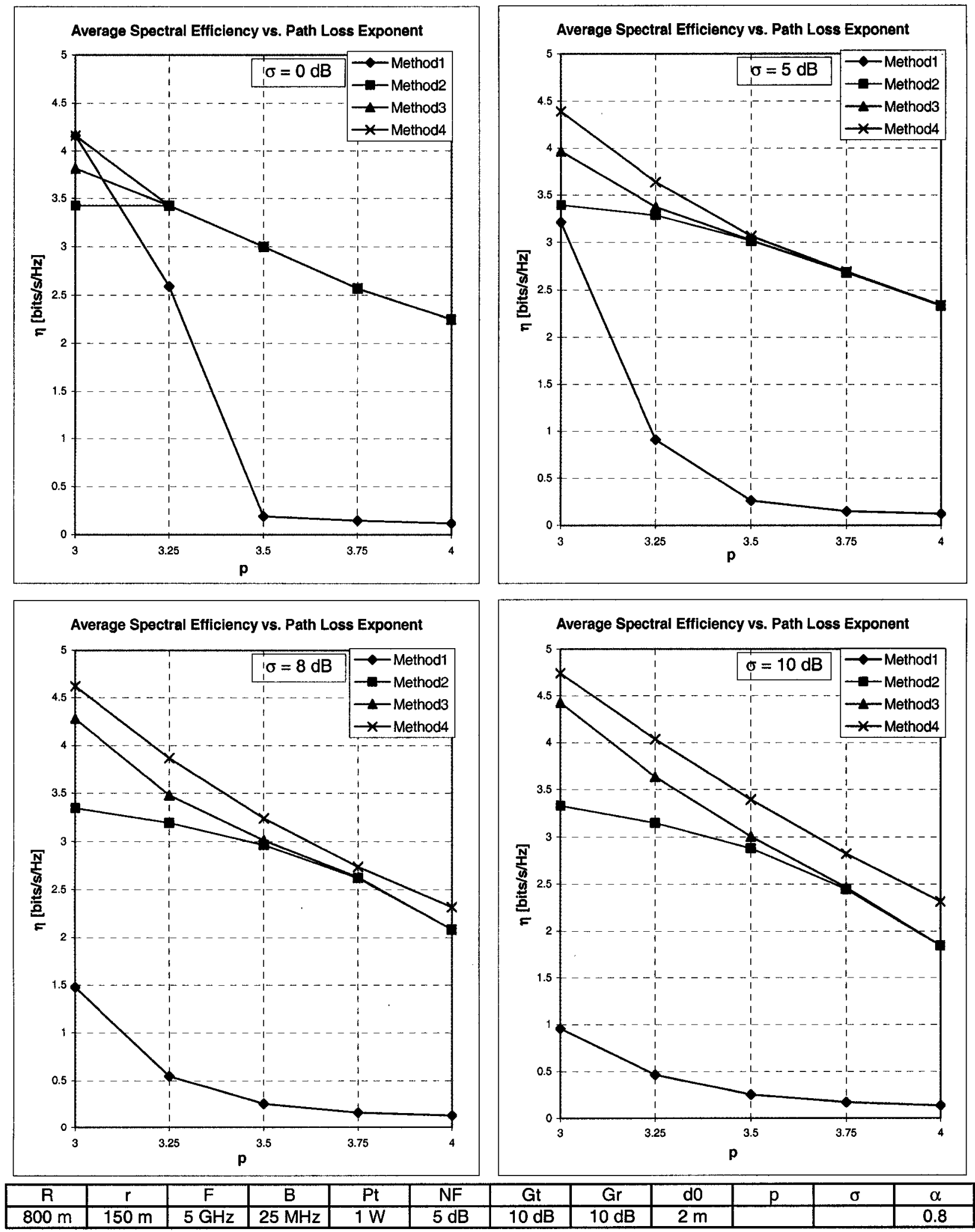

Figure 26. Average network spectral efficiency, relay service radius $150 \mathrm{~m}, \alpha=0.8$ 
We can conclude:

1. Comparing the plots in Figure 22 and Figure 23 with the ones in Figure 25 and Figure 26 we can see that for low values of the path loss exponent, the reduction factor $\alpha$ has little or no significant effect. However, for high $p$ values, the average spectral efficiency is improved significantly, by approximately $1.5 \mathrm{bit} / \mathrm{s} / \mathrm{Hz}$.

2. The Method 3 based on MHC is close to the optimal solution, achieving an average spectral efficiency within $0.5 \mathrm{bit} / \mathrm{s} / \mathrm{Hz}$ from optimal.

3. As seen in Figure 27, the increase in spectral efficiency for higher standard deviation values is less evident when the path loss for short hops is reduced. The effect of random path loss variations on spectral efficiency is partially "buried" under the improvement in spectral efficiency created by the reduced path loss.

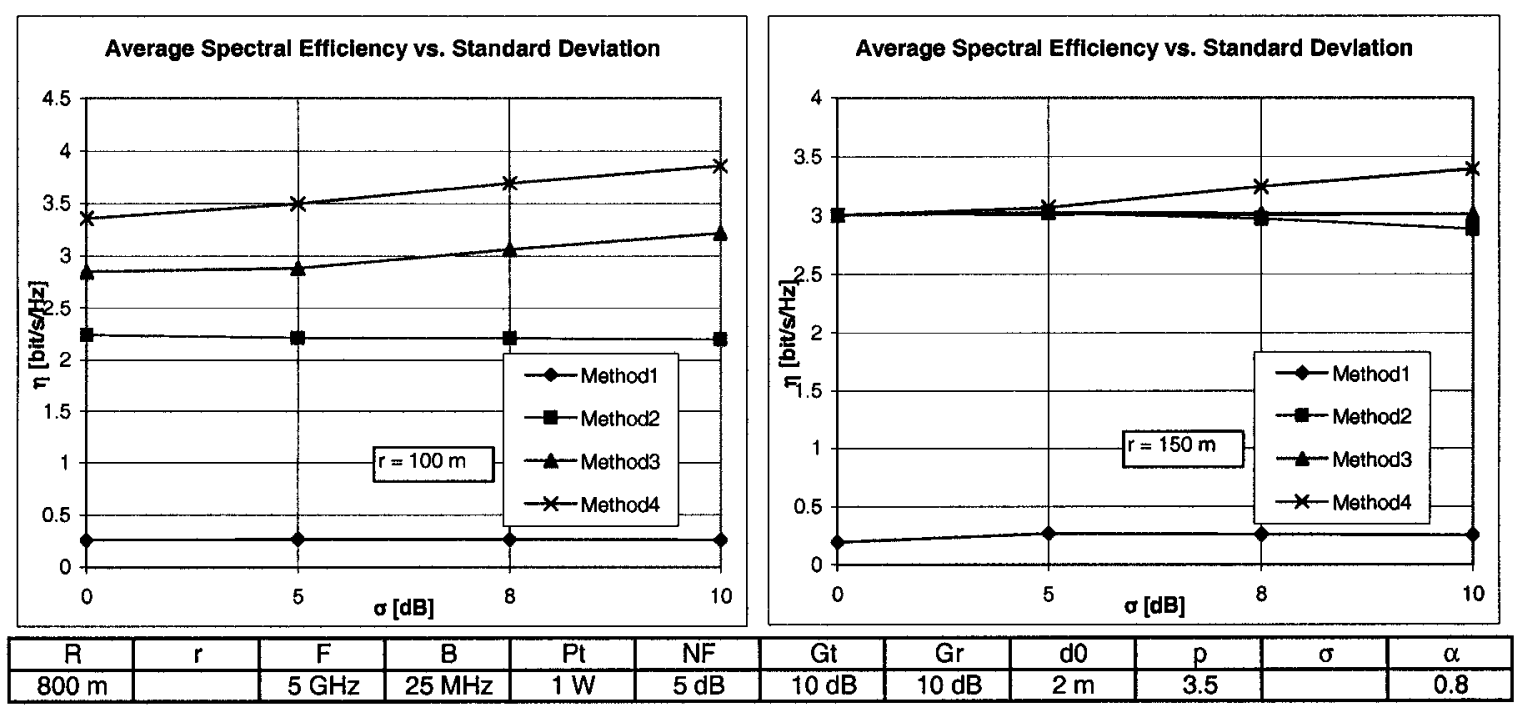

Figure 27. Average Spectral Efficiency vs. Standard deviation, $\alpha=0.8$ 


\section{CONCLUSIONS}

This section includes a summary of the dissertation, and a list of research topics which can further clarify some of the many unanswered questions on the relay based network deployment.

The work presented so far makes a strong case in favor of using relaying as an essential component of $4 \mathrm{G}$ systems. Under the initial assumption, we have considered for analysis only the feeder link between the base station and the relay providing access service to mobile station (this assumes the link BS - MT has at least two hops). Even under such "lighter" requirements we have shown that in most cases the spectral efficiency of multi-hop relaying systems outperforms the single-hop techniques, provided that specific deployment strategies for relays are followed:

- Every effort must be made to increase the inter-relay distance, and reduce the number of relays to the minimum, provided the coverage objectives to provide the required grade of service are met.

- The feeder system parameters must be chosen such that the maximum data rate can be achieved between adjacent relay locations.

- When a chain of relays is to be deployed between a source and a destination, the ideal locations are along the straight line from start to end, at equal intervals.

- The feeder system antennas should be installed within LOS of each other whenever possible

\subsection{Contributions}

In this thesis we have proven the necessity to reduce the number of relays fed from a single base station, while still meeting the service coverage objectives. The implementation of a very high throughput $4 \mathrm{G}$ wireless system using fixed relay stations presents a scalability issue from a 
radio resource costing perspective. While the amount of radio resources needed for the WAS has a constant value (due to radio resource "reuse") the amount of radio resources needed for the feeder system grows linearly with the number of relays in the cell (since no reuse is possible on the feeder system). In order to reduce this imbalance and minimize costs, one has to reduce the number of FRSs, which means employing various methods to increase the maximum service range of each FRS (cell radius).

Using Jensen's inequality, we have shown that for high SNR values, a single hop link has better spectral efficiency compared with a $n$-hop replacement; for small SNR values of the single-hop link, a more efficient $n$-hop link is possible, with the optimal locations of multi-hop regenerative relays being at equal intervals along the straight line between the source and destination. A novel quantitative criterion is developed - the Multi-Hop Criterion - which offers threshold mean SNR values below which a $n$-hop replacement could be considered over a single-hop link. We have obtained quantitative equations for the $\mathrm{MHC}$ in two sets of conditions: the first, as presented in (34), applicable when the single hop link and all intermediate hops of the multi-hop are assumed to follow the same propagation loss exponent; the second, in (39), for the scenario when the intermediate hops of the multi-hop link have smaller path loss exponent.

Using computer simulation, we have compared a routing algorithm based on the MHC, with three other routing methods: single-hop direct transmission, greedy multi-hop transmission, and exhaustive (optimal) search. We concluded the MHC method outperforms in all situations the single-hop and the greedy multi-hop algorithms, having performance (in terms of average network spectral efficiency) close to or on par with the exhaustive search method.

\subsection{Future research}

The system model used in this study relied on uniform resource allocation (power, frequency, antenna gain) to every network element. A more complex model in which resources would be 
allocated where are needed most - for example to trunk links with high traffic, or longer links and the minimization problem of its total costs, could be an interesting research topic.

Investigating and determining the exact combination of system parameters yielding the maximum net average spectral efficiency (as presented qualitatively in Figure 3) and the optimal number of relays in a BS service area (an optimization problem), would be an important step in the development of a systematic deployment strategy for cellular network with fixed relays.

Additional research into the statistical distribution of spectral efficiency for multi-hop links may bring further clarifications on the properties of cellular systems using fixed relays for multihop communications, including more in depth analysis of shadowing effects. As shown in the dissertation, the spectral efficiency statistical properties could be modeled on a reciprocal Gaussian random variable.

More refined radio channel models for communication between fixed relays are needed to fully characterize feeder systems. Most channel models currently used in literature are borrowed from mobile communication channels. Beside the differences related to the "fixed" nature of the channel (i.e., no frequency dispersion, much less Rayleigh fading), other aspects should be included, for example the conscious selection by the system designer of good relay locations with unobstructed radio links back to the servicing base station. Measurement campaigns or simulation on real high resolution GIS/ terrain databases are needed o characterize such effects, which could "skew" significantly the shadowing statistical distribution parameters. 


\section{REFERENCES}

1. H. Yanikomeroglu, Cellular multihop communications: infrastructure-based relay network architecture for $4 \mathrm{G}$ wireless systems", the 22nd Queen's Biennial Symposium on Communications (QBSC'04), 1-3 June 2004, Queen's University, Kingston, Ontario, Canada

2. R. Pabst, B.H. Walke, D. C. Schultz, P. Herhold, H. Yanikomeroglu, S. Mukherjee, H. Viswanathan, M. Lott, W. Zirwas, M. Dohler, H. Aghvami, D. D. Falconer, and G. P. Fettweis, "Relay-based deployment concepts for wireless and mobile broadband radio", IEEE Communications Magazine, September 2004

3. H. Yanikomeroglu, "Fixed and mobile relaying technologies for cellular networks", Second Workshop on Applications and Services in Wireless Networks (ASWN'02), pp. 75-81, 3-5 July 2002, Paris, France

4. ITU-R M.1645, "Framework and overall objectives of the future development of IMT-2000 and systems beyond IMT-2000", ITU-R, 2003

5. R.E. Khan, "The Organization of computer resources into a packet radio network", IEEE Transactions On Communications, Vol. COM-25, No. 1, January 1977

6. J.N. Laneman and G.W. Wornell, "Energy-efficient antenna sharing and relaying for wireless networks", Wireless Communications and Networking Conference, 2000, Volume: 1, 23-28 Sept. 2000

7. J.N. Laneman, G.W. Wornell, and D.N.C. Tse, "An efficient protocol for realizing cooperative diversity in wireless networks", IEEE International Symposium on Information Theory, 2001. Proceedings, 24-29 June 2001, Pages:294

8. J. Boyer, D. Falconer, and H. Yanikomeroglu, "Multihop Diversity in Wireless Relaying Channels", IEEE Transactions on Communications, Vol. 52, No. 10, October 2004

9. Y.-D. Lin and Yu.-C. Hsu, "Multihop cellular: a new architecture for wireless communications", IEEE INFOCOM, 2000

10. T.-C. Hou and V.O.K. Li, "Transmission range control in multihop packet radio networks", IEEE Transactions on Communications, Vol. com-34, No1, January 1986

11. V. Wong and C. Leung, "Effect of Rayleigh fading in a multihop mobile packet radio network with capture", IEEE Transactions on Vehicular Technology, Vol. 44, No. 3, August 1995

12. P. Larsson and N. Johhansson, "Multiuser diversity forwarding in multihop packet radio networks", Accepted to IEEE Wireless Communications and Networking Conference 2005 (WCNC'05) 
13. M. Lott, M. Weckerle, and M. Siebert, "Scheduling in wireless multihop networks", Proc. of International Symposium of Performance Evaluation of Computer and Telecommunication Systems (SPECTS '04), San Jose, California, 25-29. July, 2004

14. W. Mohr, R. Luder, and K.-H. Mohrmann, "Data rate estimates, range calculations and spectrum demand for new elements of systems beyond IMT-2000", The 5th International Symposium on Wireless Personal Multimedia Communications, (WPMC '02), 27-30 Oct. 2002, Pages:37 - 46 vol.1.

15. H. Bolukbasi, H. Yanikomeroglu, D. Falconer, and S. Periyalwar, "On the capacity of cellular fixed relay networks", IEEE Canadian Conference on Electrical and Computer Engineering 2004 (CCECE'04), 2-5 May 2004

16. D. Chr. Schultz, R. Pabst, M. Schmidt, M. Lott, A. Pollard, J. Ojala, P. Pellati, J. Gosteau, P. Slanina, A. Santos, and Qiliano Perez, WINNER Internal Report 3.1, IST-2003-507581

17. H. Bolukbasi, H. Yanikomeroglu, D. Falconer, and S. Periyalwar, "Feasibility of providing high data coverage in cellular fixed relay networks", World Wireless Research Forum meeting no. 12 (WWRF-12), 3-4 November 2004, Toronto, Canada.

18. G. Caire, G. Taricco, and E. Biglieri, "Bit-interleaved coded modulation," IEEE, Trans. Info. Theory, vol. 44, no. 3, pp. 927-946, May 1998.

19. T. M. Cover and J. A. Thomas, Elements of Information Theory, John Wiley and Sons, 1991.

20. A.. Leon-Garcia, Probability and Random Processes for Electrical Engineering, Pearson Education Canada, 1993.

21. T.S. Rappaport, Wireless Communication: Principles and Practice, Prentice Hall, 1996.

22. COST 231 Final Report, Chapter 4, available at http://www.lx.it.pt/cost231/final_report.htm.

23. I. Marsland, Principles of Digital Communications, Course Notes, Carleton University, 2001.

24. A. Leon-Garcia and I. Widjaja, Communication Network: Fundamental Concepts and Key Architectures, McGraw Hill, 2000. 


\section{APPENDIX 1- SIMULATION RESULTS. ROUTES}

Here, a summary of network topologies for all 4 routing methods and various simulation parameters is presented. Each diagram represents the routes generated by a simulation run, and the resulting average spectral efficiency (ASE) value corresponds with the plots in section 4.2. A blue line represents a radio link over a hop. A small red triangle denotes a receiver, while a hexagon denotes a transmitter. The analysis is done for downstream direction only; except the BS location, all locations with transmitters must also have receivers. Locations with receivers only (denoted by red triangles) are "terminal" relays which do not "feed" any other relays.

Remarks:

1. The network diagrams for Method 3 and Method 4 are adapting to propagation conditions; at one extreme (low path loss exponent) they are similar with Method 1 (direct single-hop), at the other (high path loss exponent) they are similar with Method 2 (greedy).

2. Since same shadowing is used for all 4 methods, the reader can investigate how Methods 2, 3 and 4 "hop" around paths with high shadowing. Often it can be observed how Method 3 and Method 4 provide similar or close routing solutions.

3. As expected the degree of "disorganization" of the network topologies increases with the standard deviation. 

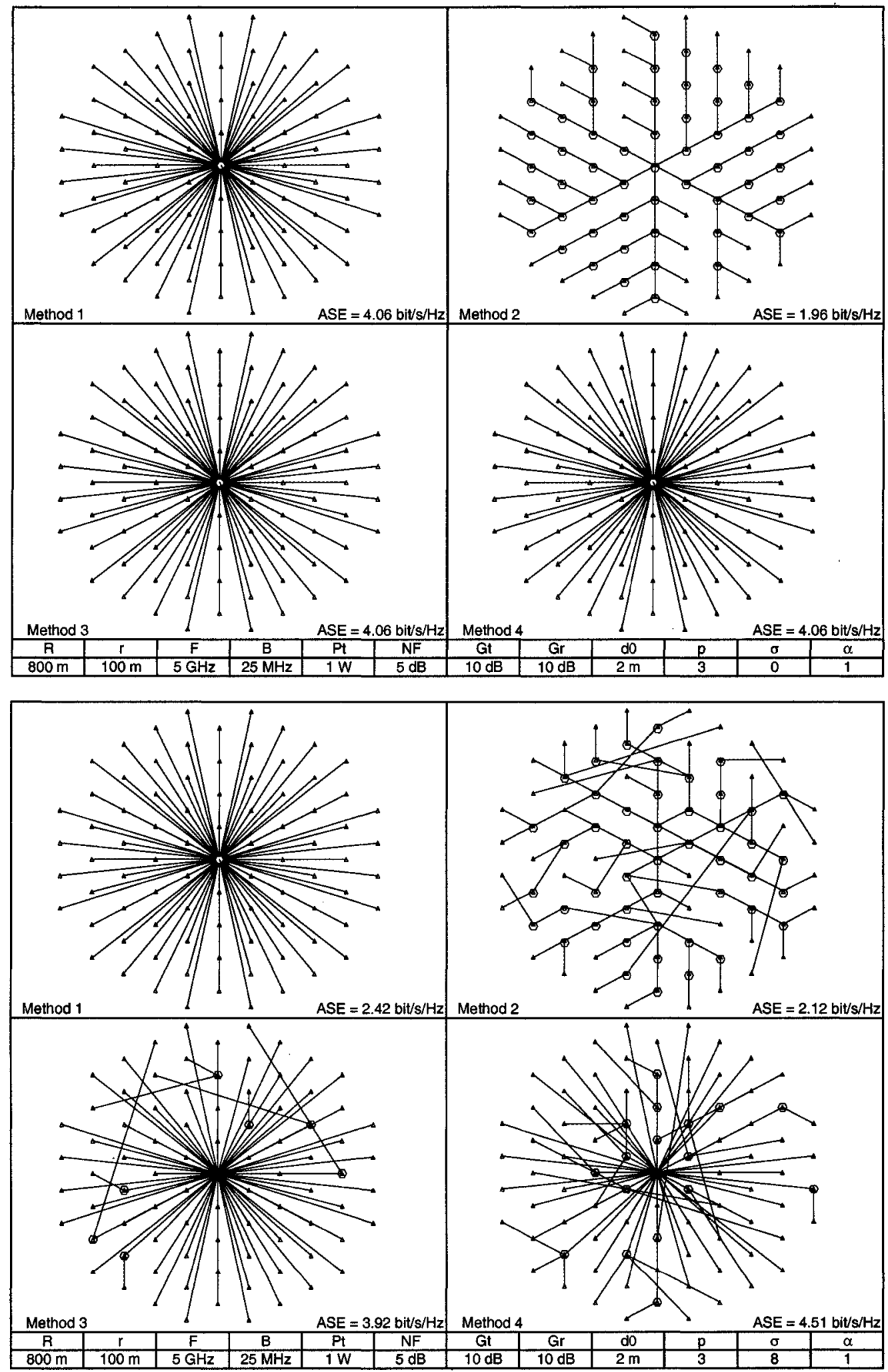

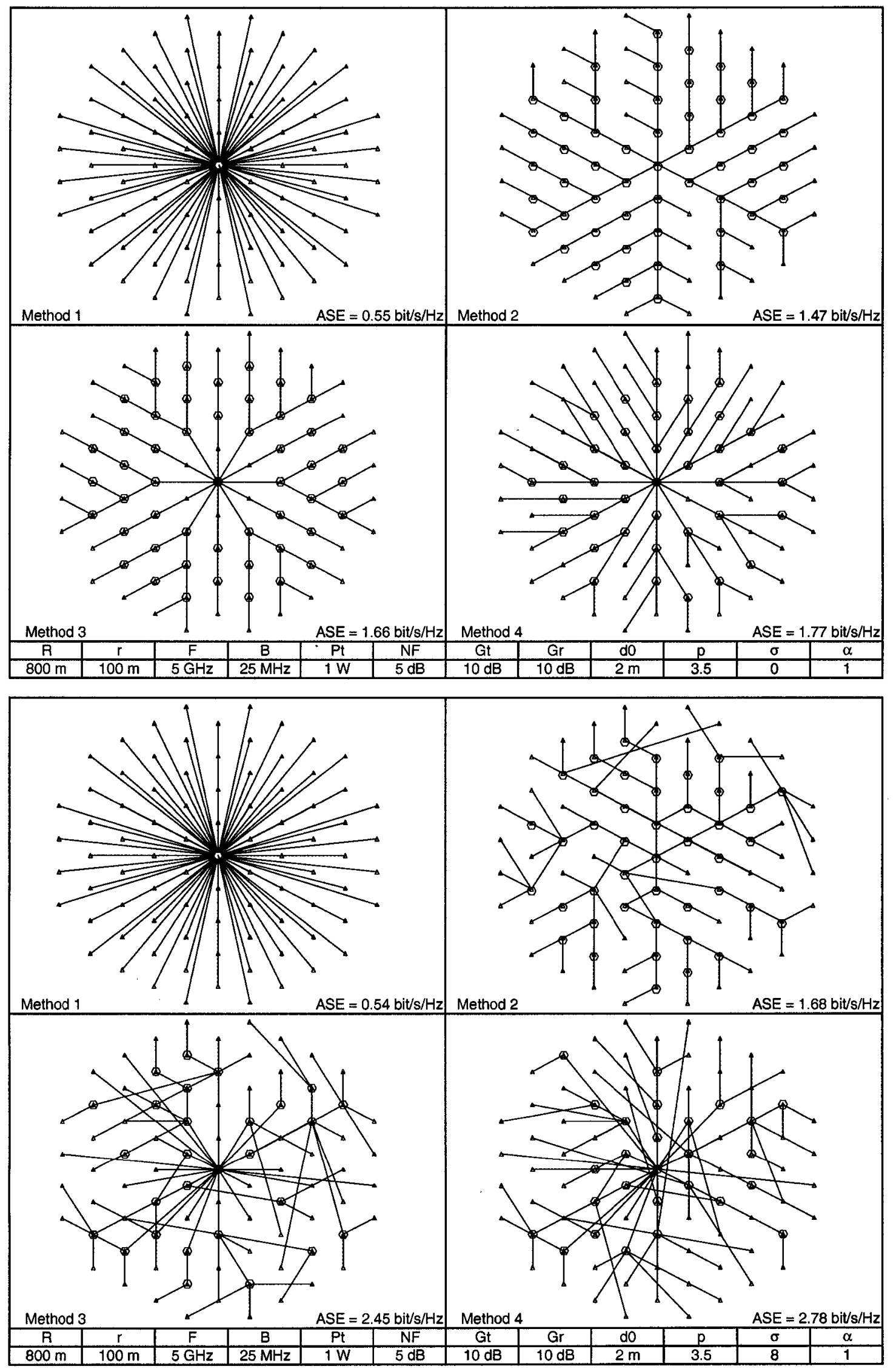

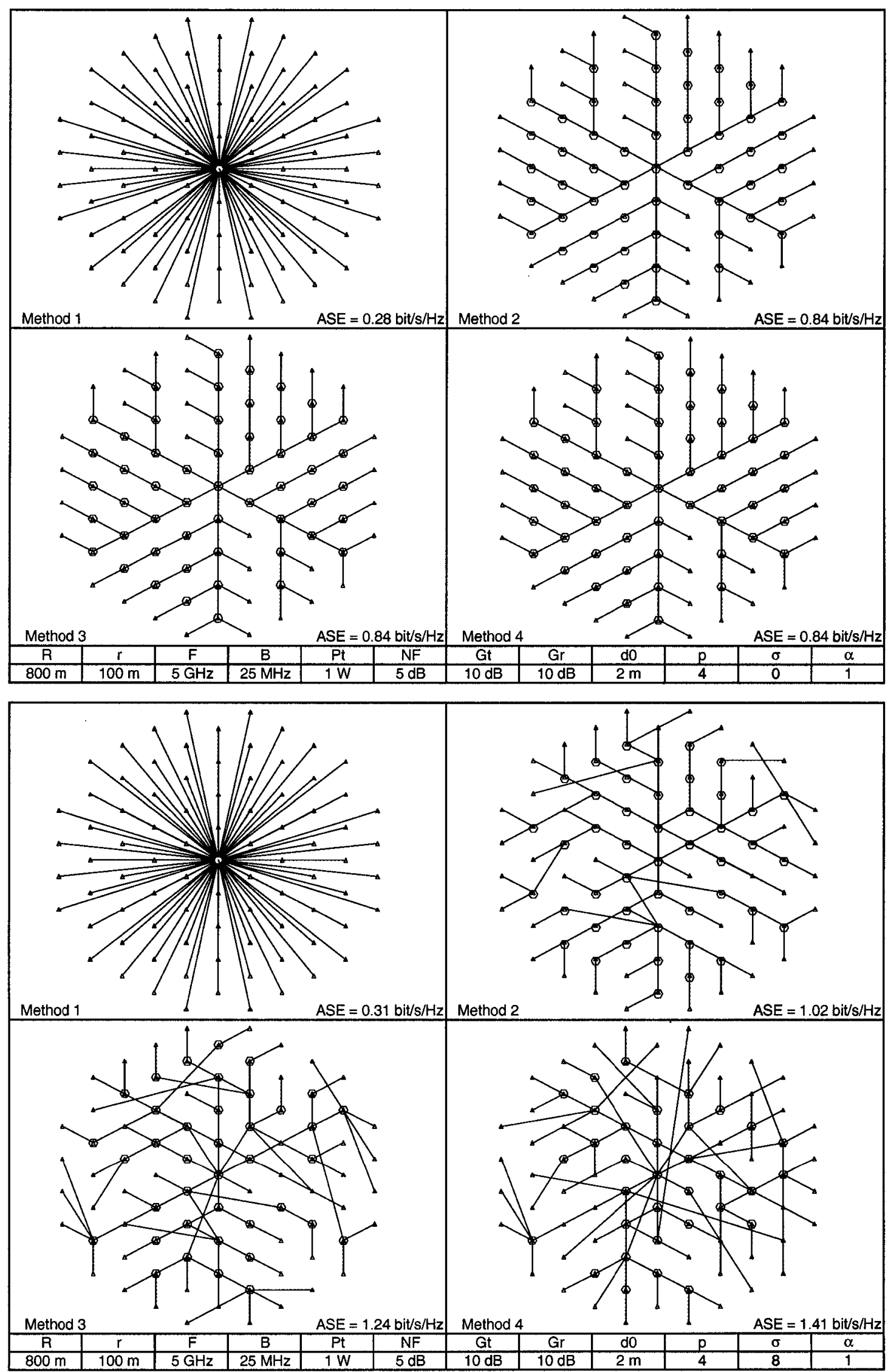

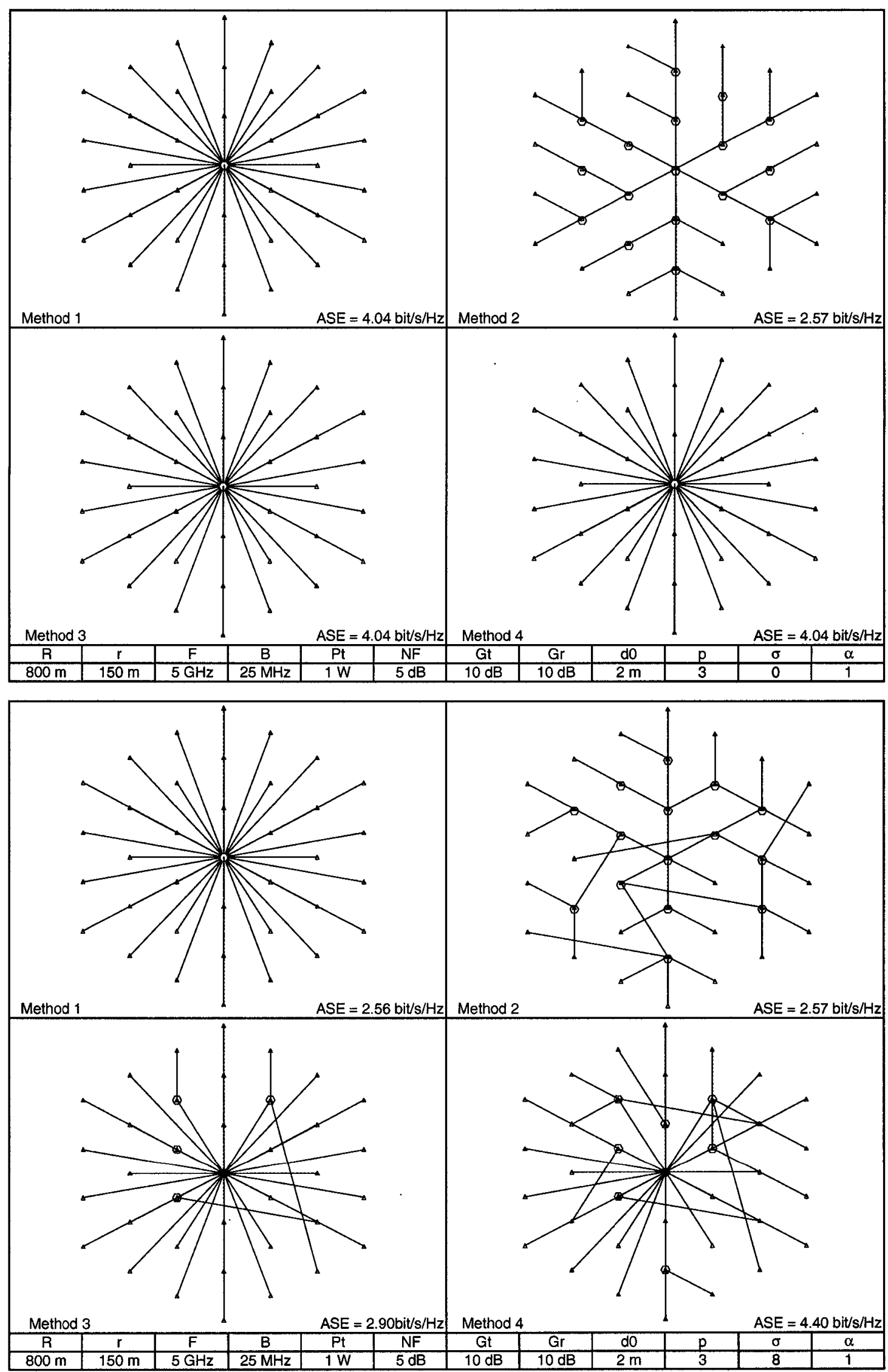

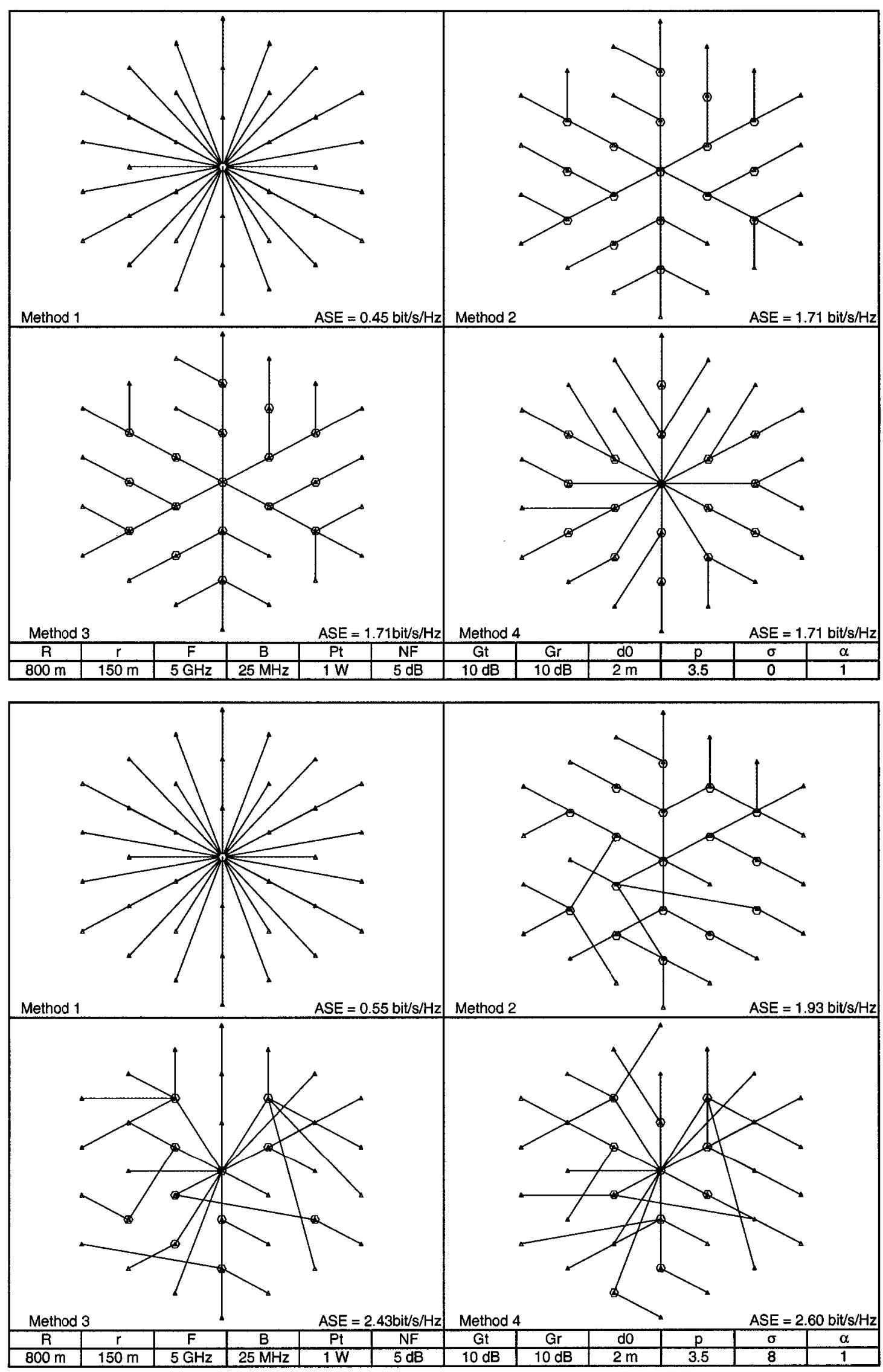

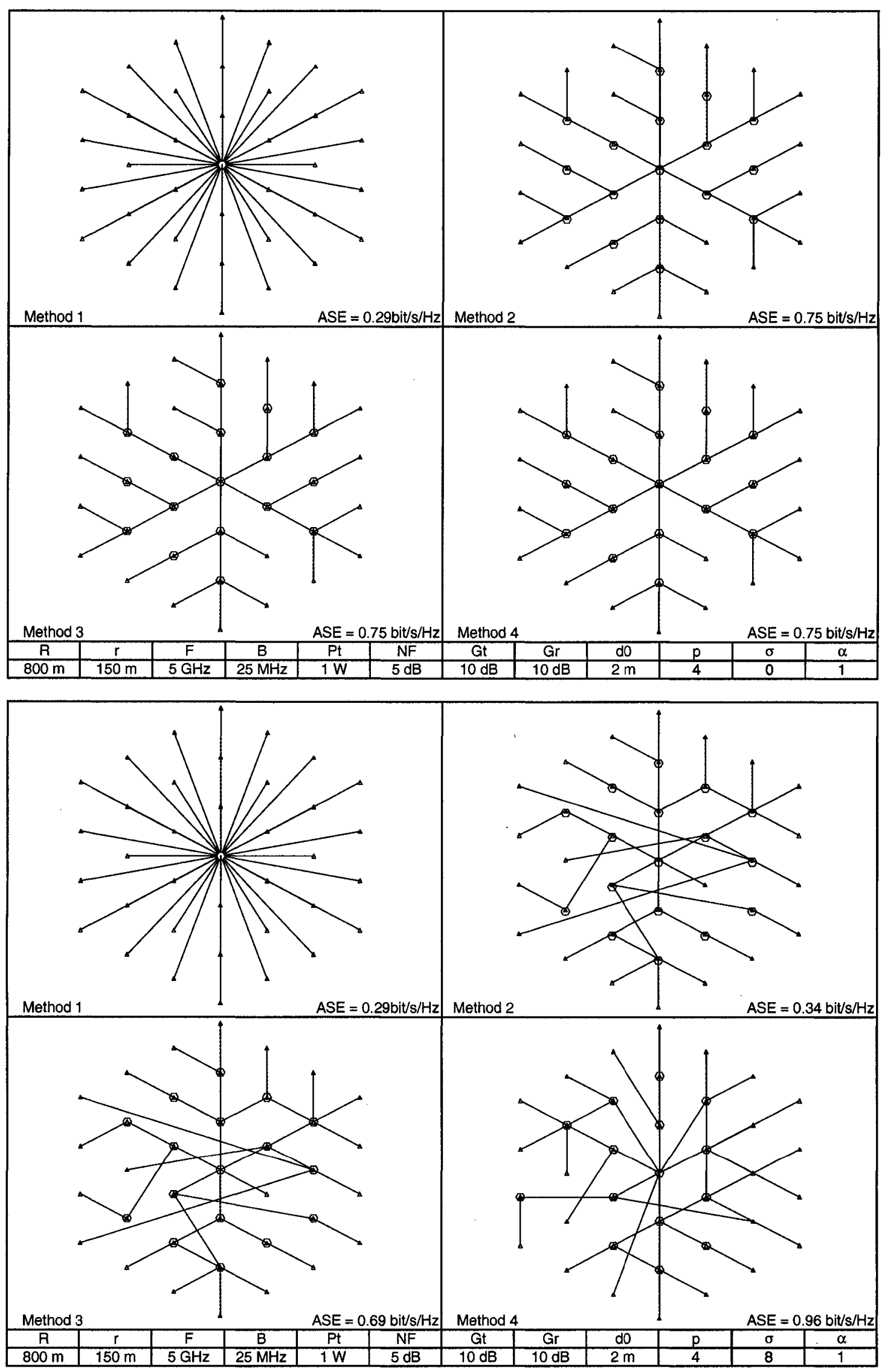

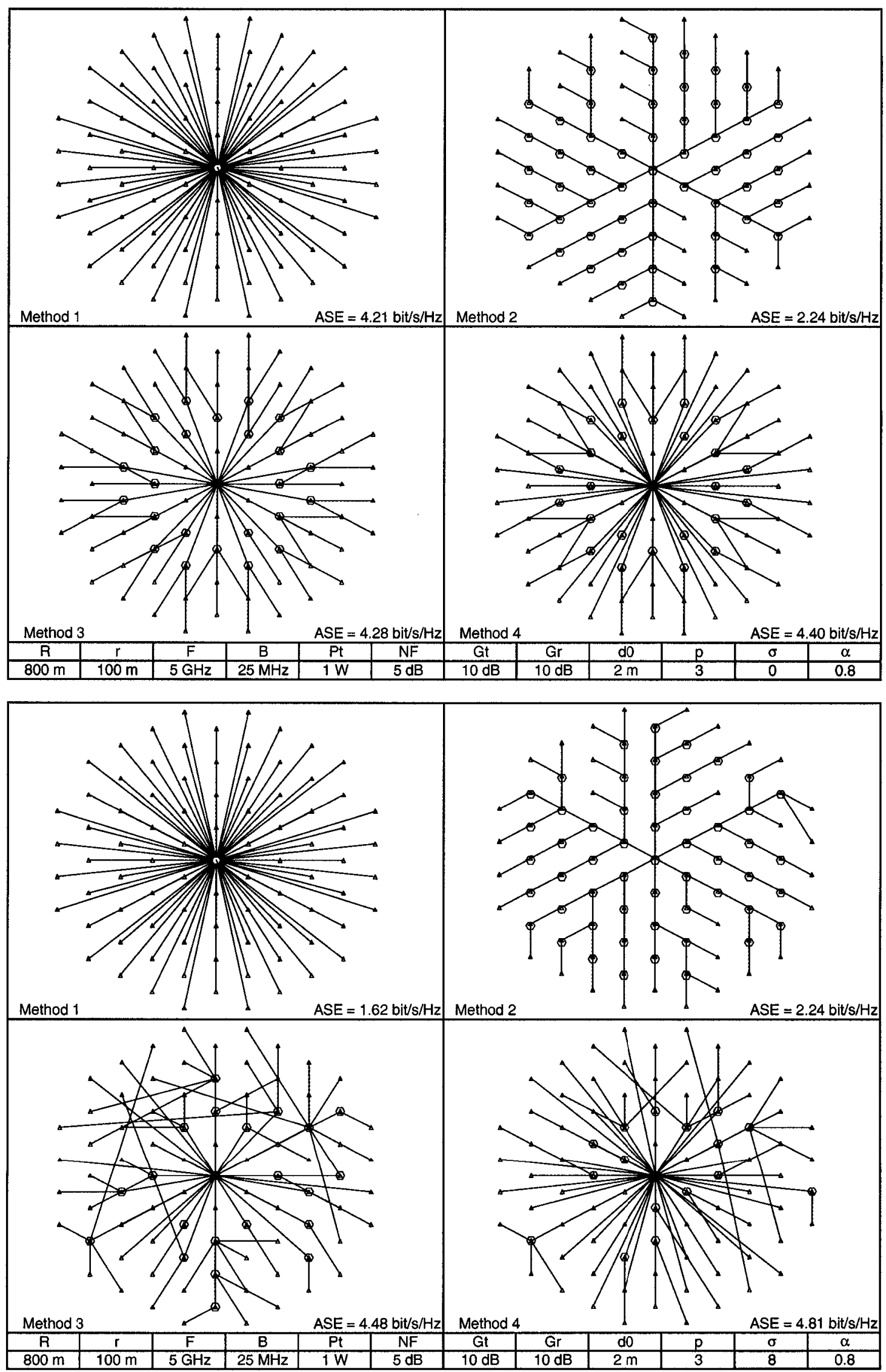

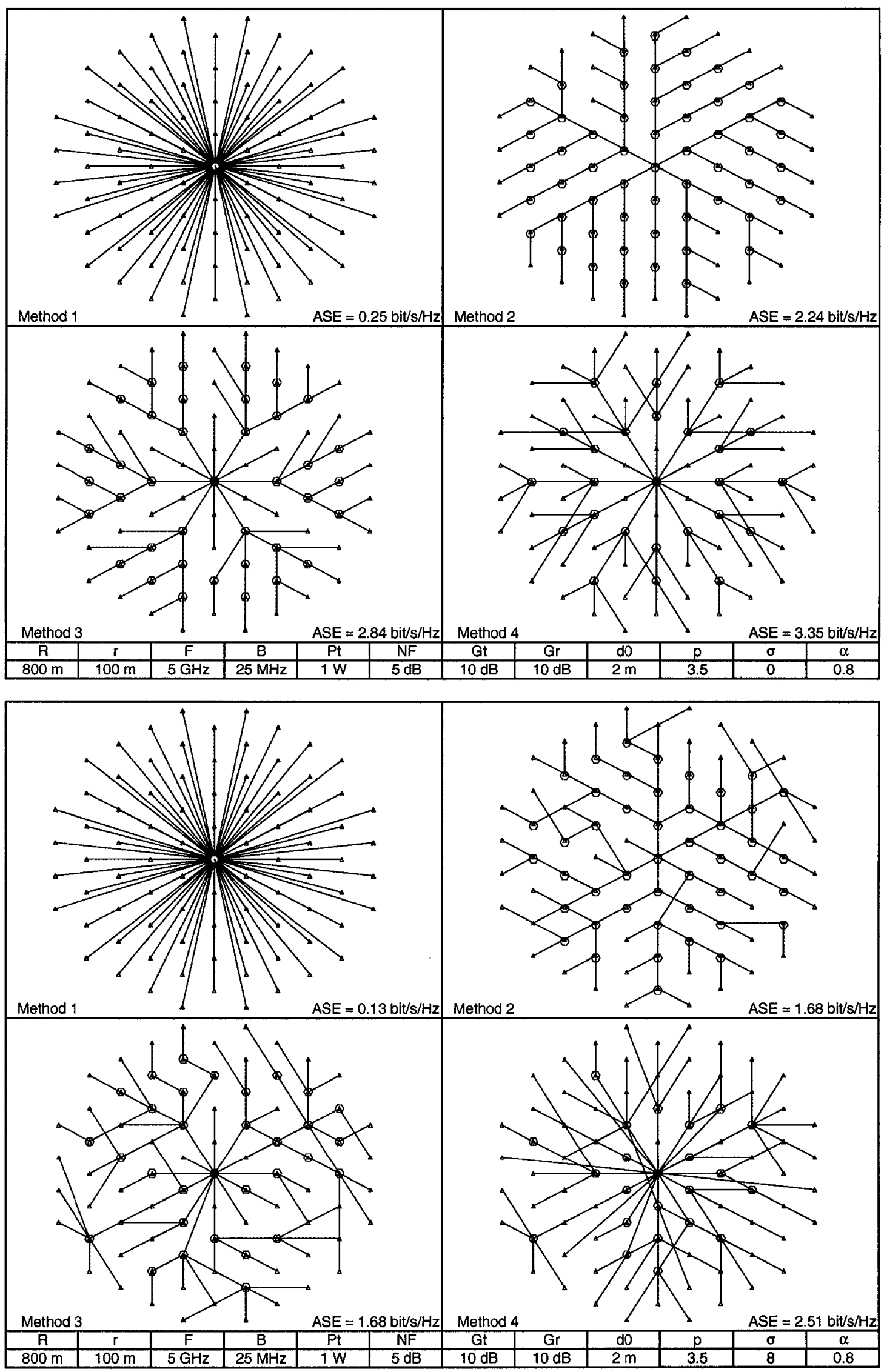

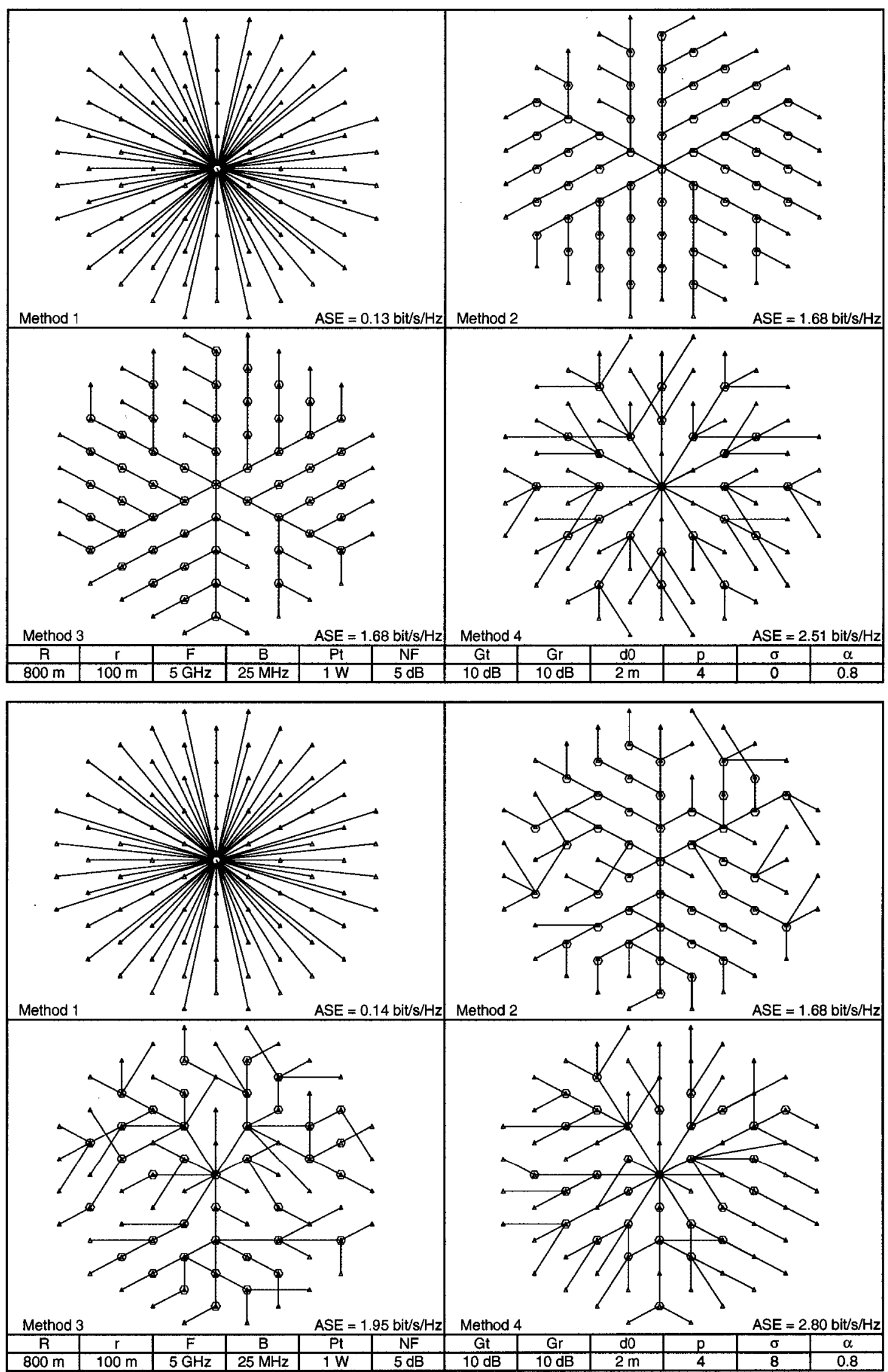

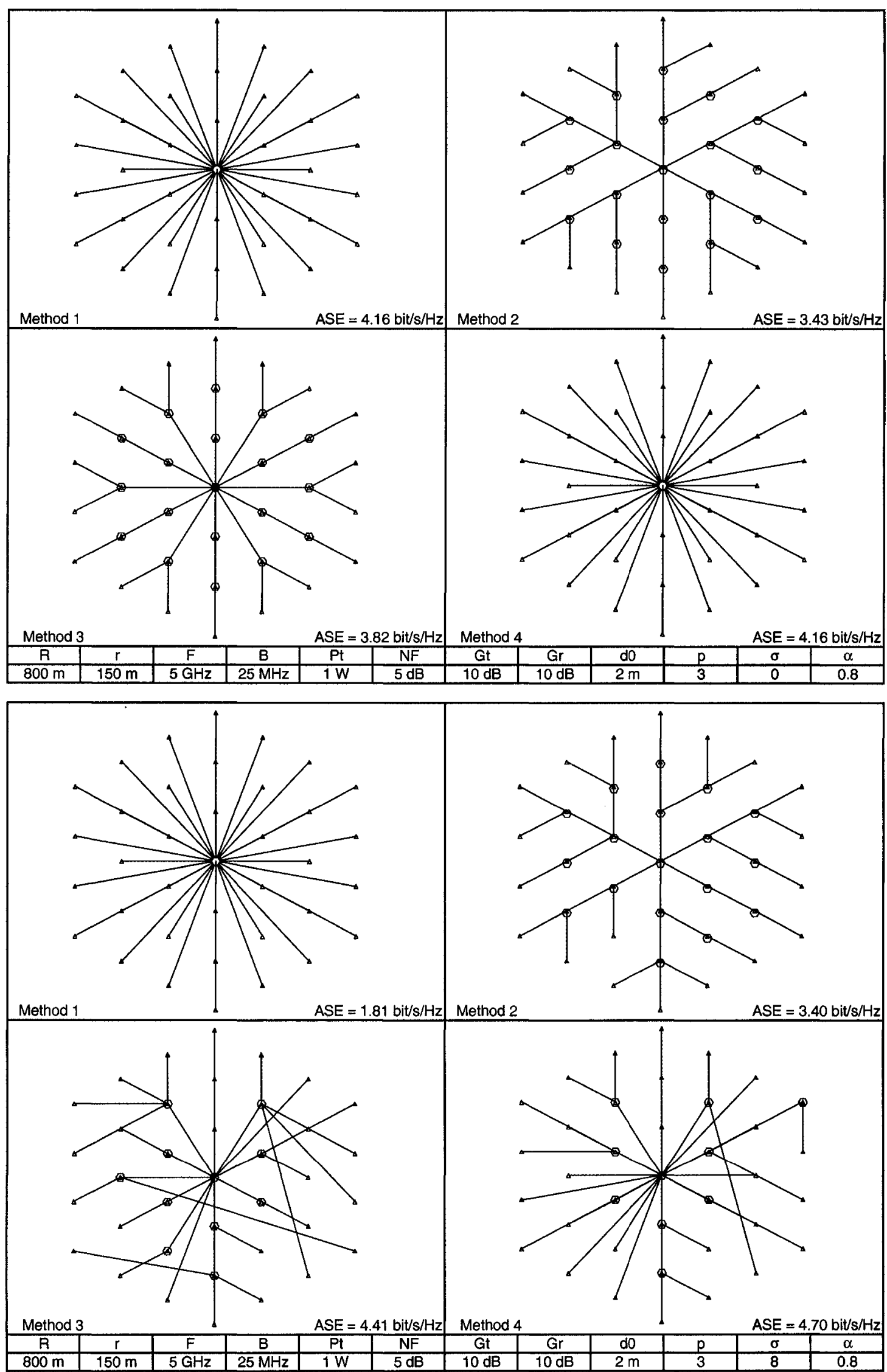

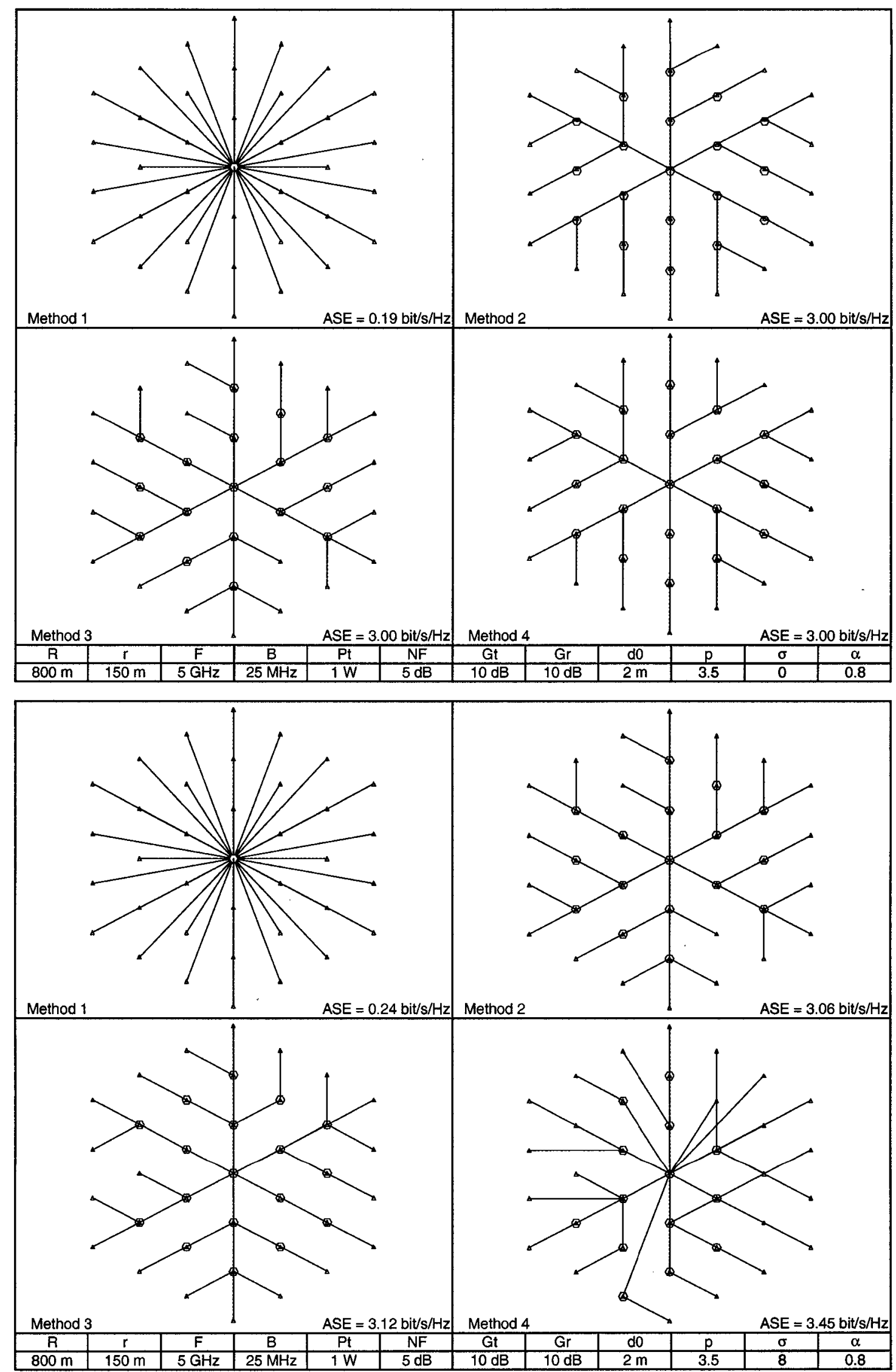

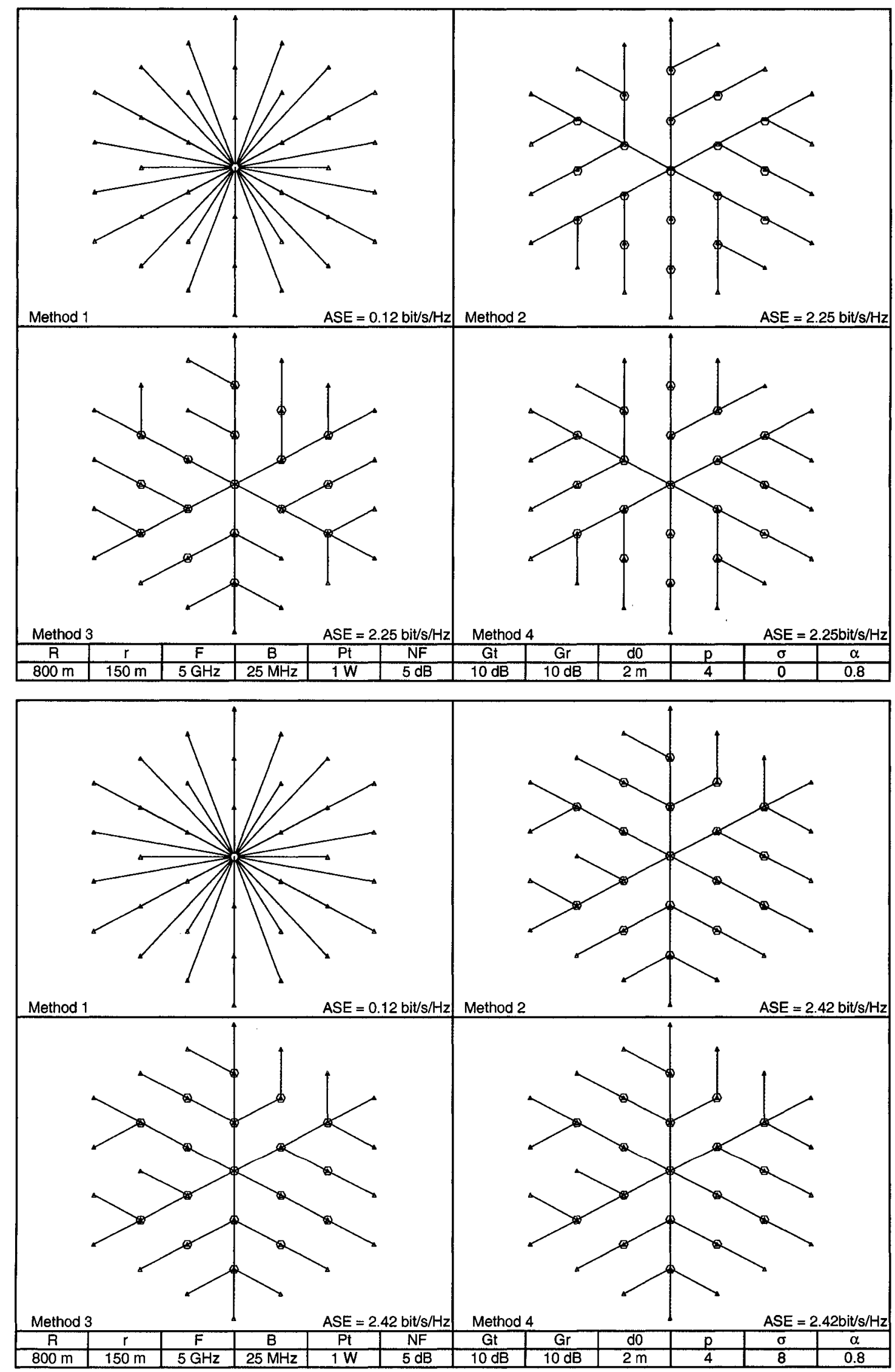